\title{
Multiplexed identification of RAS paralog imbalance as a driver of lung cancer growth
}

Rui Tang

Stanford University

Emily Shuldiner

Stanford University

Marcus Kelly

Stanford University

Christopher Murray

Stanford University

Jess Hebert

Stanford University

Laura Andrejka

Stanford University

Min Tsai

Stanford University https://orcid.org/0000-0003-4732-4259

Nicholas Hughes

Stanford University

Mitchell Parker

Fox Chase Cancer Center

Hongchen Cai

Stanford University

Yao-Cheng Li

The Salk Institute

\section{Geoffrey Wahl}

Salk Institute for Biological Studies https://orcid.org/0000-0003-3658-2279

\section{Roland Dunbrack}

Fox Chase Cancer Center

\section{Peter Jackson}

Stanford University School of Medicine https://orcid.org/0000-0002-1742-2539

\section{Dmitri Petrov}

Stanford University

Monte Winslow ( $\square$ mwinslow@stanford.edu )

Stanford University https://orcid.org/0000-0002-5730-9573 
Article

Keywords: lung cancer, KRAS mutations, CRISPR/Cas9, genome editing

Posted Date: September 17th, 2021

DOl: https://doi.org/10.21203/rs.3.rs-900548/v1

License: (c) (i) This work is licensed under a Creative Commons Attribution 4.0 International License. Read Full License 
Rui Tang ${ }^{1 *}$, Emily G. Shuldiner ${ }^{2 *}$, Marcus Kelly ${ }^{3,4}$, Christopher W. Murray ${ }^{3}$, Jess D. Hebert ${ }^{1}$, 10 Laura Andrejka ${ }^{1}$, Min K. Tsai ${ }^{1,3}$, Nicholas W. Hughes ${ }^{1}$, Mitchell I Parker ${ }^{5}$, Hongchen Cai ${ }^{1}$, Yao11 Cheng Li ${ }^{6}$, Geoffrey M. Wahl ${ }^{6}$, Roland L. Dunbrack ${ }^{5}$, Peter K. Jackson ${ }^{3,4}$, Dmitri A. Petrov ${ }^{2,3}$, and 12 Monte M. Winslow ${ }^{1,3,7 \#}$

$13{ }^{1}$ Department of Genetics, Stanford University School of Medicine, Stanford, CA, USA

$14{ }^{2}$ Department of Biology, Stanford University, Stanford, CA, USA

$15{ }^{3}$ Cancer Biology Program, Stanford University School of Medicine, Stanford, CA, USA

${ }^{4}$ Baxter Laboratories, Stanford University School of Medicine, Stanford, CA, USA

${ }^{5}$ Molecular Therapeutics Program, Institute for Cancer Research, Fox Chase Cancer Center, Philadelphia, PA, USA.

${ }^{6}$ Gene Expression Laboratory, The Salk Institute for Biological Studies, La Jolla, CA, USA

${ }^{7}$ Department of Pathology, Stanford University School of Medicine, Stanford, CA, USA 


\section{ABSTRACT}

Oncogenic $K R A S$ mutations occur in approximately $30 \%$ of lung adenocarcinoma. Despite

31 several decades of effort, oncogenic KRAS-driven lung cancer remains difficult to treat, and our

32 understanding of the positive and negative regulators of RAS signaling is incomplete. To uncover

33 the functional impact of diverse KRAS-interacting proteins on lung cancer growth in vivo, we used

34 multiplexed somatic CRISPR/Cas9-based genome editing in genetically engineered mouse models

35 with tumor barcoding and high-throughput barcode sequencing. Through a series of CRISPR/Cas9

36 screens in autochthonous lung tumors, we identified HRAS and NRAS as key suppressors of

37 KRAS $^{\mathrm{G} 12 \mathrm{D}}$-driven tumor growth in vivo and confirmed these effects in oncogenic KRAS-driven

38 human lung cancer cell lines. Mechanistically, RAS paralogs interact with oncogenic KRAS,

39 suppress KRAS-KRAS interactions, and reduce downstream ERK signaling. HRAS mutations

40 identified in KRAS-driven human tumors partially abolished this effect. Comparison of the tumor-

41 suppressive effects of HRAS and NRAS in KRAS- and BRAF-driven lung cancer models

42 confirmed that RAS paralogs are specific suppressors of oncogenic KRAS-driven lung cancer in

43 vivo. Our study outlines a technological avenue to uncover positive and negative regulators of

44 oncogenic KRAS-driven cancer in a multiplexed manner in vivo and highlights the role of RAS

45 paralog imbalance in oncogenic KRAS-driven lung cancer.

46

47

48

49

50 


\section{INTRODUCTION}

The RAS family genes $K R A S, H R A S$ and $N R A S$ are frequently mutated across cancers, and

$53 K R A S$ mutations occur in approximately $30 \%$ of lung adenocarcinomas ${ }^{1-3}$. RAS proteins are small

54 GTPases that switch between a GTP-bound active state and GDP-bound inactive state in response

55 to upstream growth signaling ${ }^{4}$. RAS proteins regulate multiple downstream signaling pathways

56 which control proliferation. Hotspot oncogenic mutations in codons 12, 13, and 61 reduce GTP

57 hydrolysis and increase the fraction of RAS proteins in the GTP-bound state, which results in

58 constitutive activation and widespread changes in RAS protein-protein interactions ${ }^{5}{ }^{6}$. These

59 changes result in hyper-activation of RAS effector pathways, driving cellular transformation and

60 tumorigenesis $^{7,8}$. Oncogenic KRAS therefore represents a key node in growth factor-induced

61 signaling and a critical target for therapeutic intervention in lung adenocarcinoma. However,

62 despite tremendous effort, the development of targeted therapies for oncogenic KRAS-driven

63 tumors has proven challenging 9 .

64 Genetic and proteomic mapping has revealed that KRAS interacts with a large network of

65 proteins $^{10,11}$. These KRAS-interacting proteins include canonical regulators and effectors, as well

66 as many proteins that remain poorly understood in the context of oncogenic KRAS-driven lung

67 cancer. Much of our understanding of RAS signaling has stemmed from diverse cellular and cell-

68 free systems ${ }^{12-14}$. Thus, while recent studies have mapped KRAS protein-protein interaction

69 networks and identified synthetic lethal interactions with oncogenic KRAS in human cell lines ${ }^{10,}$

$7011,15,16$, it remains difficult to assess the relevance of these biochemical and genetic interactions to

71 cancer growth in vivo. Genetically engineered mouse models of oncogenic KRAS-driven cancer

72 uniquely recapitulate autochthonous tumor growth and have contributed to our understanding of

73 KRAS signaling ${ }^{17}$. However, the development and use of such models has traditionally been 
74 insufficiently scalable to broadly assess modifiers of KRAS-driven tumor growth. The ability to

75 uncover functional components of RAS signaling that affect lung cancer growth in vivo in a

76 multiplexed manner would accelerate our understanding of RAS biology and could aid in the

77 development of pharmacological strategies to counteract hyperactivated KRAS.

78 To enable the analysis of genetic modifiers of lung tumor growth in vivo, we recently 79 integrated somatic CRISPR/Cas9-based genome editing with tumor barcoding and high-

80 throughput barcode sequencing (Tuba-seq) ${ }^{18-20}$. This approach allows precise quantification of the

81 effect of inactivating panels of genes of interest on lung tumor initiation and growth in a

82 multiplexed manner. By employing Tuba-seq to assess the functions of KRAS-interacting proteins

83 nominated by unbiased affinity purification/mass spectrometry (AP/MS), we show that wild-type

84 HRAS and NRAS suppress the growth of oncogenic KRAS-driven lung adenocarcinoma.

85 Competition between oncogenic KRAS and wild-type HRAS diminishes KRAS-KRAS interaction

86 and suppresses downstream signaling. In vivo screening across multiple oncogenic contexts

87 revealed that HRAS and NRAS specifically suppress the growth of tumors driven by oncogenic

88 KRAS. Our study reveals that RAS paralog imbalance is a driver of oncogenic KRAS-driven lung 89 cancer.

90

\section{RESULTS}

\section{Selection of candidate KRAS-interacting proteins to assess in vivo}

To identify putative KRAS-interacting proteins that could affect oncogenic KRAS-driven

95 lung tumor growth in vivo, we integrated previous proteomic data from AP/MS studies with gene 96 expression data from cancer cells from autochthonous mouse models (Figure 1a) ${ }^{10,21}$. We 
97 prioritized a list of candidate genes according to the probability of their protein products interacting with KRAS, their mRNA expression in mouse models of oncogenic $\mathrm{KRAS}^{\mathrm{G} 12 \mathrm{D}}$-driven lung cancer,

99 and the probability of their protein products interacting with other RAS GTPases (Figure 1b-c,

100 Figure S1a-d $)^{10,21}$. We selected 13 proteins that represent diverse aspects of RAS biology, 101 including RAS paralogs (HRAS, NRAS - which were supported by the identification of paralog102 specific peptides), RAS regulators (RASGRF2, RAP1GDS1) ${ }^{22,}$ 23, a RAS farnesyltransferase

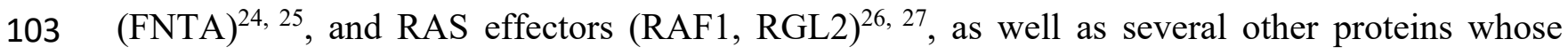
104 functions in RAS signaling are understudied. Analysis of human lung adenocarcinoma genomic 105 data showed that while most of these candidate genes trend to be more often amplified in human 106 adenocarcinoma, NRAS, HRAS, and ALDH1A1 also have deep genomic deletions (Figure S1e) ${ }^{28}$. 107 Interestingly, some of these proteins bound preferentially to either GTP- or GDP-bound KRAS, 108 while others seemed to interact with KRAS independent of its nucleotide state (Figure 1c).

\section{Identification of KRAS-interacting proteins that impact lung tumor growth in vivo}

Given that KRAS-interacting proteins could have either positive or negative effects on

112 signaling and tumor growth, we first assessed whether Tuba-seq is capable of detecting gene-

113 targeting events that have deleterious effects on tumor fitness. We initiated tumors in Kras ${ }^{L S L}$

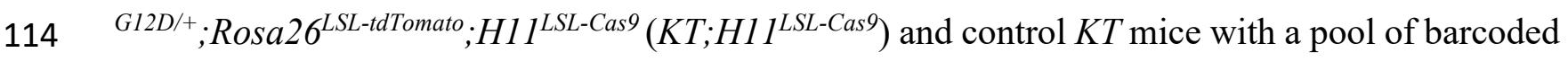
115 Lenti-sgRNA/Cre vectors encoding sgRNAs targeting two essential genes (Pcna and Rps19), a 116 known tumor suppressor $(A p c)^{20,29}$, and several inert sgRNAs (Lenti-sgEssential/Cre; Figure 117 S2a). After 12 weeks of tumor growth, we performed Tuba-seq on bulk tumor-bearing lungs and 118 quantified the number and size of tumors initiated with each Lenti-sgRNA/Cre vector (Figure 
119 S2b). By incorporating measures of tumor number and size, we could confidently identify genetic deficiencies that reduced tumor fitness (Figure S2c-g and Methods).

To quantify the impact of inactivating our panel of KRAS-interacting proteins on oncogenic $\mathrm{KRAS}^{\mathrm{G} 12 \mathrm{D}}$-driven lung tumor growth in vivo, we generated a pool of barcoded LentisgRNA/Cre vectors targeting the genes that encode these proteins, as well as sgInert control vectors (Lenti-sgKrasIP/Cre; Figure 1d). Given the importance of farnesylation in KRAS localization and signaling, sgRNA targeting Fnta served as a control for KRAS dependency ${ }^{30,31}$. We initiated tumors with the Lenti-sgKrasIP/Cre pool in KT;H11 $1^{\text {LSL-Cas } 9}$ and $K T$ mice and calculated metrics of tumor size and number after 12 weeks of tumor growth (Figure 1e). To our surprise, inactivation of the Kras paralogs Hras and Nras had the most dramatic effect on tumor growth. Inactivation of Candl also increased tumor size, while deletion of several genes including Fnta, Nme2, Raplgdsl, and Aldhla decreased tumor size and/or number, suggesting reduced cancer cell fitness (Figure 1f and S3a-d).

Given the fundamental importance of the p53 tumor suppressor in oncogenic KRAS-driven lung cancer, as well as previous data suggesting crosstalk between RAS and p53 signaling ${ }^{19,32,33}$, we determined whether p53 deficiency changed the impact of inactivating KRAS-interacting proteins on tumor growth. We initiated tumors with the Lenti-sgKrasIP/Cre pool in Kras ${ }^{L S L-}$ ${ }^{\text {G12D/+ }} ; \operatorname{Rosa} 26^{\text {LSL-tdTom }} ; \operatorname{Trp} 53^{\text {floxfflox }} ; H 11^{\text {LSL-tdTom }}\left(\right.$ KT;Trp5 $\left.3^{\text {flox/flox }} ; H 11^{\text {LSL-Cas9 }}\right)$ mice and performed Tuba-seq after 12 weeks of tumor growth (Figure 1e). The effects of inactivating each gene encoding a KRAS-interacting protein on tumor size, tumor number, and overall tumor burden were generally consistent between the p53-proficient and -deficient settings (Figure 1g, Figure S3e-h). Notably, the inactivation of either Hras or Nras also significantly increased growth of p53deficient tumors (Figure 1g, Figure S3e). Collectively, these results suggest that HRAS and 
142 NRAS are tumor suppressors within in vivo models of oncogenic KRAS-driven lung cancer, while

143 several other KRAS-interacting proteins, including CAND1, ALDH1A, and NME2, have less

144 consistent effects on tumor growth between p53-proficient and -deficient backgrounds (Figure

145 S3e-h).

146

147 Validation of HRAS and NRAS as suppressors of oncogenic KRAS-driven lung tumor 148 growth

149 To further validate the effect of inactivating six top candidate genes (Hras, Nras, Cand1, 150 Aldhla, Fnta, and Nme2) on oncogenic KRAS-driven tumor growth in vivo and confirm that these

151 results are driven by on-target effects, we generated and barcoded three Lenti-sgRNA/Cre vectors

152 targeting each gene. To contextualize the effect of Hras and Nras inactivation on lung tumor

153 growth relative to established tumor suppressors we included vectors targeting three established

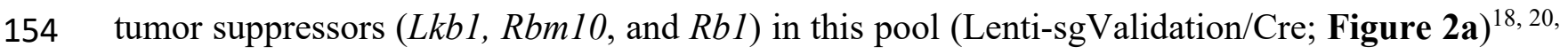

15534 . We initiated tumors with the Lenti-sgValidation/Cre pool in $K T ; H 11^{L S L \text {-Cas } 9}$ and $K T$ mice and 156 assessed metrics of tumor initiation and growth 12 weeks after tumor initiation (Figure 2 b-c). 157 Targeting Fnta with all three sgRNAs consistently reduced growth fitness, while the impact of 158 inactivating Aldhla and Nme2 was more variable (Figure 2d, Figure S4). Most importantly, all 159 three sgRNAs targeting Hras and all three sgRNAs targeting Nras significantly increased tumor 160 growth (Figure 2d-e, Figure S4b). Notably, Hras inactivation increase tumor growth to a similar 161 extent as inactivation of the $R b 1$ and $R b m 10$ tumor suppressors (Figure 2d, Figure S4b). These 162 results suggest a potentially pivotal role for wild-type HRAS and NRAS in constraining oncogenic 163 KRAS-driven lung tumor growth in vivo. 
In addition, we validated the tumor-suppressive function of HRAS and NRAS in oncogenic

165 KRAS-driven lung tumor growth by initiating tumors in $K T ; H 11^{L S L-C a s 9}$ mice with individual 166 sgInert-, sgHras- and sgNras-containing Lenti-sgRNA/Cre vectors (Figure 2f). Inactivation of 167 either Hras or Nras increased tumor growth as assessed by direct fluorescence and histological 168 analyses (Figure 2g-k). Collectively, these results suggest that RAS paralogs constrain the growth 169 of oncogenic $\mathrm{KRAS}^{\mathrm{G} 12 \mathrm{D}}$-driven lung cancer growth.

\section{HRAS and NRAS can be growth-suppressive in human lung cancer cells}

To assess the relevance of HRAS and NRAS as tumor suppressors in human lung cancer,

173 we tested the function of HRAS and NRAS in oncogenic KRAS-driven human lung 174 adenocarcinoma cell lines. Previous genome-scale CRISPR/Cas9 screens revealed that 175 inactivating these genes was generally detrimental to cancer cell line growth under standard culture 176 conditions (Figure S5a) ${ }^{10,35}$. Interestingly, HRAS and NRAS suppressed the growth of oncogenic $177 \mathrm{KRAS}^{\mathrm{G} 12 \mathrm{~S}}$-driven A549 cells and of several oncogenic KRAS-driven lung cancer cell lines when 178 grown in 3D culture conditions, suggesting that these genes can function as tumor suppressors in 179 certain contexts (Figure S5b-c) ${ }^{10,15}$. To further assess the functions of HRAS and NRAS in 180 oncogenic KRAS-driven human adenocarcinoma cell lines, we performed gain and loss of function 181 studies on $\mathrm{H} 23\left(\mathrm{KRAS}^{\mathrm{G} 12 \mathrm{C} /+}\right)$ and $\mathrm{H} 727\left(\mathrm{KRAS}^{\mathrm{G} 12 \mathrm{~V} /+}\right)$ cells under growth factor restricted growth 182 conditions. We inactivated $H R A S$ and $N R A S$ using CRISPR/Cas9 and generated variants with 183 doxycycline-inducible wild-type $H R A S$ re-expression. Inactivation of $H R A S$ or $N R A S$ in oncogenic 184 KRAS-driven cells increased cell growth when cells were grown with limited serum and increased 185 clonal growth potential when cells were grown in anchorage-independent conditions (Figure 3a, 186 c, d). Conversely, re-expression of HRAS in these $H R A S$-null cells impaired proliferation and 
187 clonal growth (Figure 3b, e, f). H23 cells with inactivated $H R A S$ or $N R A S$ also formed larger and more proliferative tumors after intravenous and subcutaneous transplantation (Figure 3g-k, Figure S6). These results demonstrate that wild-type HRAS and NRAS can also function as tumor suppressors in oncogenic KRAS-driven human lung cancer cells in vitro and in vivo. This consistency between human cell culture and autochthonous mouse models further suggests that HRAS and NRAS are tumor suppressors in oncogenic KRAS-driven lung adenocarcinoma.

\section{Inactivation of RAS paralogs increases signaling downstream of oncogenic KRAS}

Wild-type KRAS has been shown to be tumor-suppressive in multiple experimental models

of oncogenic KRAS-driven cancer, likely due to its ability to interact with and antagonize

197 oncogenic KRAS ${ }^{36-38}$. We have demonstrated that wild-type HRAS and NRAS suppress oncogenic 198 KRAS $^{\mathrm{G} 12 \mathrm{D}}$-driven lung cancer growth in vivo. Thus, to further explore the molecular mechanism 199 driving this effect, we initially assessed whether HRAS and NRAS alter signaling downstream of 200 oncogenic KRAS. We initially performed pERK immunohistochemistry on lung tumors initiated 201 with Lenti-sgRNA/Cre vectors containing sgInert, sgHras or sgNras in KT;H11 ${ }^{\text {LSL-Cas } 9}$ mice. 202 Inactivation of HRAS or NRAS increased the number of pERK-positive cells in KRAS ${ }^{\mathrm{G} 12 \mathrm{D}}$-driven 203 lung cancer (Figure 4a, Figure S7a). Subcutaneous tumors from transplanted H23 cells with 204 inactivated $H R A S$ or $N R A S$ also contained more pERK-positive cells when compared to tumors 205 from transplanted wildtype (sgSAFE) H23 cells (Figure 4b, Figure S7b). In addition, sorted 206 cancer cells from KT;H1 $1^{L S L-C a s 9}$ mice with lung tumors initiated with Lenti-sgHras/Cre also had 207 greater pERK and pAKT compared to those from tumors initiated with Lenti-sgInert/Cre (Figure 208 4c, Figure S7c). Inactivation of either Hras or Nras in mouse (HC494) or human (H23 and 209 HOP62) oncogenic KRAS-driven cell lines increased ERK phosphorylation, while their effects on 
210 AKT phosphorylation was more cell context dependent (Figure 4d-e, Figure S7d-e). Conversely,

211 re-expression of wild-type HRAS in HRAS-null H23 and HOP62 human lung cancer cells reduced

212 ERK phosphorylation again with cell context dependent effect on AKT phosphorylation (Figure

213 4f, Figure S7f). Previous publications have shown that inactivating wild-type KRAS increases

214 sensitivity to MEK inhibitors ${ }^{37,39}$. Consistent with these studies, we found that inactivation of

$215 H R A S$ in $\mathrm{H} 23$ cells increased sensitivity to the MEK inhibitor trametinib while re-expression of

216 HRAS made cells more resistant (Figure 4g, h). These data suggest that inactivation of HRAS or

$217 N R A S$ hyper-activates MAPK-ERK signaling in KRAS mutant cancer cells ${ }^{40-42}$.

\section{RAS paralogs suppress oncogenic KRAS-KRAS interaction}

220 RAS proteins interact and form functional clusters on membranes to efficiently recruit

221 downstream effectors ${ }^{43-45}$. Whether RAS proteins form dimers or oligomers through direct

222 interactions or through close physical proximity is debated within the field ${ }^{16,46-48}$. We next

223 assessed whether HRAS and NRAS "interact" with KRAS without attempting to distinguish direct

224 from proximity-driven interactions. AP/MS data suggest that all three RAS proteins are able to

225 interact with their paralogs, supporting the existence of heterotypic RAS-RAS interactions (Figure

226 5a). To assess the ability of RAS paralogs to interact with oncogenic KRAS ${ }^{\mathrm{G} 12 \mathrm{D}}$, we adapted a

227 luminescent reporter system (ReBiL2.0 system), which relies on luciferase complementation to 228 quantify RAS-RAS interactions in living cells ${ }^{16}$ (Figure 5b). Through expression of wild-type 229 KRAS, HRAS, or NRAS in KRAS ${ }^{\mathrm{G} 12 \mathrm{D}}-\mathrm{KRAS}^{\mathrm{G} 12 \mathrm{D}}$ interaction reporter cells and control reporter

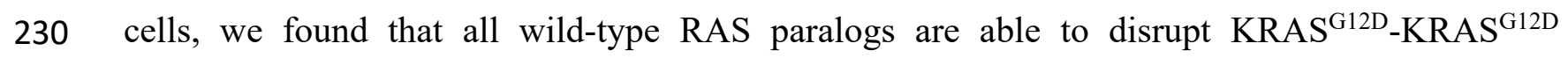
231 interactions (Figure 5c, Figure S10a). 
233 Patient-derived HRAS ${ }^{\text {T50M }}$ and HRAS ${ }^{\text {R123C }}$ mutations impair interaction of HRAS with 234 oncogenic KRAS and abrogate its tumor suppressive function

235 Our findings suggest that the tumor-suppressive function of wild-type HRAS is mediated

236 by competitive interactions with oncogenic KRAS, therefore we hypothesized that there could be

$237 H R A S$ mutations in human tumors with oncogenic $K R A S$ that impair this interaction. To evaluate

238 this possibility, we analyzed data from AACR's Genomics Evidence Neoplasia Information

239 Exchange (GENIE). Mutations in $H R A S$ were rare (pan-cancer frequency of non-synonymous

240 mutations was $1.32 \%)$ and about half (0.57\%) were oncogenic mutations in codons 12,13 or 61

241 that occurred in samples lacking oncogenic $K R A S$ (Figure S8a). We did, however, identify

242 multiple rare non-oncogenic $H R A S$ mutations in oncogenic KRAS containing lung

243 adenocarcinomas and tumors of other types (Figure 5d, Figure S8). To test whether these mutants

244 lack the ability to interact with oncogenic KRAS, we used the ReBiL2.0 system. We measured the

245 ability of four of these $H R A S$ mutants, as well as a control Y64A mutant that has been suggested

246 to reduce HRAS-HRAS dimerization ${ }^{47}$, to inhibit KRAS $^{\mathrm{G} 12 \mathrm{D}}-\mathrm{KRAS}^{\mathrm{G} 12 \mathrm{D}}$ interactions. We

247 identified two HRAS mutants, T50M and R123C, that are unable to reduce KRAS ${ }^{\mathrm{G} 12 \mathrm{D}}$-KRAS ${ }^{\mathrm{G} 12 \mathrm{D}}$

248 interactions (Figure 5e, Figure S10b). Interestingly, both HRAS ${ }^{\mathrm{T} 50}$ and HRAS ${ }^{\mathrm{R} 123}$ are located

249 close to the predicted HRAS-KRAS ${ }^{\mathrm{G} 12 \mathrm{D}}$ interface involving the $\alpha 4$ and $\alpha 5$ helices (Figure 5f,

250 Figure S9). R123 is involved in an intrachain salt bridge with residue E143, which also participates

251 in the RAS-RAS interface. Mutation to cysteine results in an uncompensated charge on E143,

252 which may destabilize the RAS-RAS interaction. These findings are consistent with a model in

253 which wild-type RAS paralogs competitively interacts with oncogenic KRAS and thus suppress

254 KRAS $^{\mathrm{G} 12 \mathrm{D}}-\mathrm{KRAS}^{\mathrm{G} 12 \mathrm{D}}$ interactions. 
Previous publications have shown that different RAS proteins preferential bind to RAF

256 proteins and other RAS effectors and thus could function differently in their downstream

257 signaling ${ }^{10,50}$. Re-analysis of HRAS and NRAS AP/MS datasets suggests that GTP-bound HRAS

258 is more similar in its low binding affinity to RAF effectors as GDP-bound rather than GTP-bound

259 KRAS and NRAS (Figure 5g) ${ }^{10}$. To test our hypothesis that the disruption of KRAS ${ }^{\mathrm{G} 12 \mathrm{D}}$ -

260 KRAS $^{\mathrm{G} 12 \mathrm{D}}$ interaction by HRAS suppresses downstream oncogenic signaling, we re-expressed 261 wild-type HRAS, HRAS ${ }^{\mathrm{Y} 64 \mathrm{~A}}$, or the two novel patient-derived HRAS ${ }^{\mathrm{T} 50 \mathrm{M}}$ and $\mathrm{HRAS}^{\mathrm{R} 123 \mathrm{C}}$ mutants

262 in $H R A S$-null lung cancer cells. Re-expression of wild-type HRAS, but not any of the three 263 mutants, reduced ERK phosphorylation and proliferation (Figure 5h-i, Figure S10c). These

264 results further suggest that RAS paralog imbalance alters oncogenic KRAS signaling via 265 oncogenic KRAS-wildtype RAS paralog interaction and thus is a driver of lung cancer growth.

267 HRAS and NRAS are specific suppressors of oncogenic KRAS-driven lung cancer growth

268 Our in vivo data demonstrate that HRAS and NRAS function as tumor suppressors, and 269 our cell culture results suggest that these effects may be mediated by interaction of these RAS 270 paralogs with oncogenic KRAS. If the tumor-suppressive mechanism by which HRAS and NRAS

271 is mediated through interactions with oncogenic KRAS, then these genes should not be tumor 272 suppressors in lung adenocarcinoma in which activation of the RAS/RAF/MEK signaling pathway 273 occurs downstream of KRAS. To test this directly in autochthonous tumors, we initiated tumors 274 with a sub-pool of barcoded lenti-sgRNA/Cre vectors (Lenti-sgMultiGEMM/Cre) in mouse 275 models of oncogenic KRAS-driven and oncogenic BRAF-driven lung cancer (Figure 6a). In 276 addition to vectors targeting Hras and Nras, this pool contained vectors targeting several known 277 tumor suppressors $(A p c, R b m 10$, and $C d k n 2 a)$ and other KRAS-interacting proteins (Aldh1a, 
278 Nme2), as well as control vectors (Figure 6a). We initiated tumors with the Lenti279 sgMultiGEMM/Cre pool in $K T$ and $K T ; H 11^{L S L-C a s 9}$ mice as well as in BrafT;H1 $1^{L S L-C a s 9}$ mice 280 which contain a Cre-regulated allele of oncogenic $\mathrm{BRAF}^{\mathrm{V} 618 \mathrm{E}}$ (the mouse equivalent of $\left.281 \mathrm{BRAF}^{\mathrm{V} 600 \mathrm{E}}\right)(\text { Figure 6b) })^{51} .15$ weeks after tumor initiation BrafT;H1 $1^{\text {LSL-Cas } 9}$ mice has greater

282 overall tumor burden than KT;H1 $1^{L S L-C a s 9}$ mice (Figure 6c-d). Analysis of the distribution of 283 sgInert tumor sizes in the two models using Tuba-seq showed that oncogenic BRAF-driven tumors 284 were larger than oncogenic KRAS-driven tumors (median sizes of $\sim 3500$ cells and $\sim 1000$ cells, 285 respectively). The two distributions had similar maximum tumor sizes, suggesting that the 286 increased tumor burden is driven by a shift towards larger tumors of relatively uniform size which 287 is consistent with previous results (Figure 6e-f) $)^{51}$.

288 Our Tuba-seq data also allowed us to compare the impact of the CRISRP/Cas9 inactivated 289 genes across oncogenic contexts. Importantly, while inactivation of Hras or Nras increased the 290 growth of oncogenic KRAS-driven lung tumors, inactivation of Hras or Nras had no effect on the 291 growth of oncogenic BRAF-driven lung cancer (Figure 6g, Figure S11d-e). These results were 292 consistent for both Lenti-sgRNA/Cre vectors targeted each gene. The known tumor suppressor 293 genes assayed (Apc, Cdkn2a, and $R b m 10)$ generally retained their growth-suppressive effects in 294 the BRAF-driven model, suggesting that the abrogation of effect observed for Hras and Nras is 295 not due to some generic inability of additional alterations to increase BRAF-driven lung tumor 296 growth (Figure 6h, Figure S11d-e). Thus, HRAS and NRAS function as specific suppressors of 297 oncogenic KRAS-driven tumor growth in vivo. Assessing the impact of genomic alterations on the growth of lung cancer driven by distinct 299 oncogenes was illuminating in two other regards. First, we identify instances of oncogene-tumor 300 suppressor epistasis (e.g., Apc inactivation has a greater effect on BRAF-driven lung cancer 
301 whereas Rbm10 inactivation has a greater effect on KRAS-driven lung cancer) (Figure 6h, Figure

302 S11d-e). Thus, the consequences of inactivating tumor suppressor pathways can depend on the 303 oncogenic context. Second, inactivation of Nme2, Fnta, and Aldhla reduced initiation and growth 304 of both oncogenic KRAS-driven and oncogenic BRAF-driven lung cancer, suggesting that they 305 are generally required for optimal lung cancer growth in vivo (Figure S11). Thus, our paired 306 screens not only localized the effect of Hras and Nras inactivation, but also highlighted the value 307 of this approach in uncovering alterations that have effects within or across oncogenic contexts.

\section{DISCUSSION}

Oncogenic KRAS-driven lung cancer is a leading cause of cancer-related deaths. However,

311 despite the identification of oncogenic RAS almost half a century ago, the functions of many RAS-

312 interacting proteins remain largely unknown. By integrating AP/MS data from human cancer cells

313 with somatic cell CRISPR/Cas9-editing in autochthonous mouse models, we assess the functional

314 impact of inactivating a panel of KRAS-interacting proteins on lung cancer in vivo in a multiplexed

315 manner. Our results support a model in which heterotypic interactions between RAS paralogs

316 suppress oncogenic KRAS-driven lung cancer growth.

All RAS family proteins, HRAS, NRAS and KRAS (including both the KRAS4A and

318 KRAS4B splice isoforms), have been reported to form dimers and nanoclusters ${ }^{16,} 46-48$.

319 Importantly, both in vitro and in vivo studies suggest that KRAS-KRAS interactions are required

320 for effector protein activation, cellular transformation, and optimal tumor growth ${ }^{45}$. Furthermore,

321 oncogenic KRAS-wild-type KRAS interactions influence lung cancer initiation, progression, and

322 therapeutic sensitivity ${ }^{37}$. Multiple lines of evidence, including oncogenic KRAS copy number gain

323 and loss of the wild-type $K R A S$ allele in human tumors, as well as functional studies in mouse 
324 models, suggest that wild-type KRAS is tumor-suppressive (also called "RAS allelic imbalance"),

325 although the exact role of wild-type KRAS in lung cancer is still debated ${ }^{3,38,41,52,53}$. Recent data

326 also suggest that interactions among $\mathrm{H}-, \mathrm{N}$ - and KRAS occur, thus raising the question of the roles

327 of wild-type HRAS and NRAS in oncogenic KRAS-driven cancer ${ }^{10,11,16}$.

328 In this study, we identified wild-type HRAS and NRAS as potent KRAS-specific tumor

329 suppressors that interact with oncogenic KRAS, disrupt KRAS-KRAS interactions, and suppress

330 RAS/MAPK signaling. Inactivation of $H R A S$ or $N R A S$ in the context of oncogenic KRAS led to

331 an increase in downstream MAPK signaling (Figure 4). The impact of RAS paralog imbalance

332 extends beyond lung cancer and KRAS codon 12 mutations. Germline Hras deletion increases the

333 development of Kras-driven pancreatic cancer, skin papilloma, and carcinogen induced KRAS ${ }^{\mathrm{Q} 1}$

334 lung cancer ${ }^{53-55}$. Interestingly, we also identified two rare, patient-derived HRAS mutations,

335 HRAS $^{\mathrm{T} 50 \mathrm{M}}$ and HRAS ${ }^{\mathrm{R} 123 \mathrm{C}}$, which are incapable of disrupting KRAS clustering, and would

336 therefore likely confer fitness advantages to oncogenic KRAS-driven cancer. These results suggest

337 that modulating RAS protein interactions, such as by skewing the stoichiometry of oncogenic to

338 wild-type RAS or forcing inter-paralog competition, could lead to novel therapeutic strategies.

339 However, the dynamics of intracellular RAS interactions, as well as the importance of these 340 mutations in oncogenesis requires further study.

341 Given the complexity of RAS signaling, other non-mutually exclusive mechanisms by

342 which RAS paralogs could reduce oncogenic KRAS-driven cancer growth should be considered.

343 For example, it has been reported that upstream regulators, such as SOS1, could bridge the

344 interaction between oncogenic and wild-type RAS ${ }^{56}$. GDP-bound wild-type HRAS and NRAS

345 could also compete with oncogenic KRAS for upstream guanine nucleotide exchange factors and

346 thus reduce RAS signaling ${ }^{57}$. In addition, although we provide evidence that inactivation of Hras 
347 and Nras has no impact on oncogenic BRAF-driven lung cancer, it is possible that they could 348 compete with oncogenic KRAS for other BRAF-independent downstream effectors. Whether 349 HRAS and NRAS also function through these alternative routes, and how different mechanisms 350 are synchronized to execute their tumor-suppressive functions, will require additional 351 investigation.

352 The National Cancer Institute "RAS Pathway V2.0", contains more than 200 proteins 353 known or suspected to be involved in RAS signaling. Characterizing the role of these proteins in 354 tractable in vivo models of RAS-driven cancer remains a challenge. Our study outlines a 355 technological avenue to study KRAS-specific signaling components in a multiplexed manner. By 356 harnessing the power of Tuba-seq, we were able to quantify the tumor suppressive and promoting 357 effects of more than a dozen putative RAS pathway genes simultaneously, highlighting the 358 function of HRAS and NRAS as tumor suppressors. Furthermore, by performing paired screens in 359 oncogenic KRAS-driven and oncogenic BRAF-driven mouse lung cancer models, we localized 360 the growth suppressive effects of these RAS paralogs to lung cancer driven specifically by 361 oncogenic KRAS. Our study thus demonstrates the feasibility of performing in vivo genetic 362 interaction screening, and the power of such an approach to provide insight into the mechanisms 363 of tumor suppression. Future studies of this type should enable a more quantitative understanding 364 of the role of RAS pathway components in RAS-driven oncogenicity. 
FIGURE LEGEND:

371 Figure 1. Multiplexed identification of KRAS-interacting proteins that impact KRAS ${ }^{\text {G12D_ }}$

372 driven lung cancer growth in vivo.

373 a. Candidate mediators of KRAS-driven lung tumor growth were identified on the basis of their

374 interactions with GTP- and GDP-locked Kras in multiple AP/MS-based protein-protein interaction

375 screens and their expression in a mouse model of Kras-driven lung adenocarcinoma.

376 b. Selected KRAS-interacting proteins interact with either GTP- or GDP-locked KRAS (shown as

377 NSAF in A549 cells) and their homolog is expressed in KRAS ${ }^{\mathrm{G} 12 \mathrm{D}}$-driven lung cancer (shown as 378 TPM).

379 c. Bubble plot of two AP/MS experiments with GTP- and GDP-locked mutant GTPases as baits 380 (rows), showing the enrichment of selected candidate KRAS-interacting proteins (columns). Dark 381 borders indicate FDR $<0.05$.

382 d. Schematic of tumor initiation with a pool of barcoded Lenti-sgRNA/Cre vectors (Lenti383 sgKrasIP-Pool/Cre). The lentiviral pool includes four Inert sgRNAs that are either non-targeting 384 (NT) or target a functionally inert locus (Neo1-3, targeting NeoR in the $R 26^{L S L-t d T o m a t o}$ allele). Each 385 barcoded lentiviral vector contains an sgRNA, Cre, and a two-component barcode composed of an 386 sgRNA identifier (sgID) and a random barcode (BC). This design allows inactivation of multiple 387 target genes in parallel followed by quantification of the resulting tumor size distributions through 388 high-throughput sgID-BC sequencing.

389 e. Tumors were initiated in cohorts of $K T, K T ; H 11^{L S L \text {-Cas } 9}$ and $K T ; p 53^{\text {flox fllox }} ; H 11^{L S L-C a s 9}$ mice 390 through intratracheal delivery of Lenti-sgKrasIP-Pool/Cre. Tuba-seq was performed on each 391 tumor-bearing lung 12 weeks after initiation, followed by analyses of sgID-BC sequencing data to 392 characterize the effects of inactivating each gene. 
393 f. Tumor sizes at indicated percentiles for each sgRNA relative to the size of sgInert-containing 394 tumors at the corresponding percentiles in $K T ; H 11^{\text {LSL-Cas } 9}$ mice. Genes are ordered by $95^{\text {th }}$ 395 percentile tumor size, with sgInerts on the left. sgInerts are in gray, and the line at $y=1$ indicates 396 no effect relative to sgInert. Error bars indicate $95 \%$ confidence intervals. Percentiles that are 397 significantly different from sgInert (two-sided FDR-adjusted $p<0.05$ ) are in color. Confidence 398 intervals and P-values were calculated by bootstrap resampling.

399 g. Comparison of $95^{\text {th }}$ percentile tumor size for each sgRNA relative to the size the $95^{\text {th }}$ percentile 400 tumor size of sgInert-containing tumors in KT;H1 $1^{\text {LSL-Cas } 9}$ mice versus $K T ; p 53^{\text {floxfllox }} ; H 11^{\text {LSL-Cas } 9}$ 401 mice. Error bars indicate 95\% confidence intervals calculated by bootstrap resampling.

403 Figure 2. HRAS and NRAS are potent suppressors of KRAS ${ }^{\text {G12D }}$-driven lung cancer growth 404 in vivo

405 a,b. A pool of barcoded Lenti-sgRNA/Cre vectors (Lenti-sgValidation/Cre) targeting candidate 406 mediators of KRAS-driven lung tumor growth identified in the initial KRAS-interacting protein 407 Tuba-seq screen was used to initiate tumors in validation cohorts of KT and KT;H1 $1^{\text {LSL-Cas } 9}$ mice. 408 This lentiviral pool includes four Inert sgRNAs, as well as sgRNAs targeting $L k b 1, R b 1$, and $409 \mathrm{Rbm} 10$ as tumor suppressor controls. Each candidate gene from the initial screen is targeted with 410 three sgRNAs. Tumors were initiated through intratracheal delivery of Lenti-sgValidation/Cre, 411 and Tuba-seq was performed on each tumor-bearing lung 12 weeks after initiation, followed by 412 analyses of sgID-BC sequencing data to characterize the effects of inactivating each gene (b).

413 c. Fluorescence images of representative lung lobes 12 weeks after tumor initiation. Scale bars $=$ $4145 \mathrm{~mm}$. Lung lobes are outlined with a white dashed line. 
415 d. Tumor sizes at indicated percentiles for each sgRNA relative to the size of sgInert-containing 416 tumors at the corresponding percentiles in $K T ; H 11^{\text {LSL-Cas } 9}$ mice. Genes are ordered by $95^{\text {th }}$ 417 percentile tumor size, with sgInerts on the left. Note that $\operatorname{sg} L k b 1$ is plotted on a separate scale 418 to facilitate visualization of sgRNAs with lesser magnitudes of effect. Dashed line indicates no 419 effect relative to sgInert. Error bars indicate 95\% confidence intervals. 95\% confidence intervals 420 and P-values were calculated by bootstrap resampling. Percentiles that are significantly different 421 from sgInert $(2$-sided FDR-adjusted $\mathrm{p}<0.05)$ are in color.

422 e. Targeting Hras and Nras significantly increases mean tumor size relative to sgInerts, assuming 423 a log-normal distribution of tumor sizes (LNmean). Error bars indicate 95\% confidence intervals 424 calculated by bootstrap resampling.

425 f. Schematic of tumor initiation with individual Lenti-sgRNA/Cre vectors. Mouse number and titer 426 of the lentiviral vectors are indicated.

427 g. Representative fluorescence images of lungs from $K T ; H 11^{L S L-C a s 9}$ mice after tumor initiation 428 with Lenti-sgRNA/Cre vectors as indicated. Scale bar $=5 \mathrm{~mm}$.

429 h. Representative H\&E images of lungs from $K T ; H 11^{L S L-C a s 9}$ mice after tumor initiation with Lenti$430 \mathrm{sgRNA} /$ Cre vectors as indicated. Tumor area (percentage of total lung area) from each mouse is 431 shown as Mean $\pm \mathrm{SD} .{ }^{*}: \mathrm{p}<0.05$; Scale bar $=5 \mathrm{~mm}$.

432 i. Tumor burden in $K T ; H 11^{L S L-C a s 9}$ mice with tumors initiated with Lenti-sgRNA/Cre vectors as 433 indicated. Each dot represents relative tumor area (percentage of total lung area) from one mouse. $434 * \mathrm{p}<0.05$

435 j. Representative BrdU staining images of lungs from $K T ; H 11^{\text {LSL-Cas } 9}$ mice after tumor initiation 436 with Lenti-sgRNA/Cre vectors as indicated. Number of Brdu ${ }^{\text {pos }}$ cells per field is shown as Mean \pm 437 SD. $* *: \mathrm{p}<0.01 ;$ Scale bar $=100 \mu \mathrm{m}$. 
438 k. Quantification of proliferation cells in $K T ; H 11^{L S L-C a s 9}$ mice with tumors initiated with Lenti$439 \operatorname{sgRNA} /$ Cre vectors as indicated. Each dot represents a tumor. $* *: \mathrm{p}<0.01$

441 Figure 3. Wildtype HRAS or NRAS constrain the growth of human KRAS-driven cancer 442 cell lines.

443 a. Inactivation of wild type HRAS or NRAS increases growth of KRAS-mutant H23 (G12C) and $444 \mathrm{H} 727(\mathrm{G} 12 \mathrm{~V})$ cells. Wildtype (sgSAFE) or HRAS- or $N R A S$-knockout cells were seeded in 96 well 445 plates and cultured under limited serum (1\%). Cell numbers were measured via CCK8 assay. 446 Points are Mean \pm SD of 12 wells normalized to Day 0. **: $\mathrm{p}<0.01$

447 b. Re-expression of wild type HRAS suppresses proliferation of HRAS-null H23 and H727 cells. 448 TRE-HRAS cells were seeded in 96 well plates and cultured under limited serum (1\%) with or 449 without $50 \mathrm{ng} / \mathrm{ml}$ Doxycycline (Dox) and cell numbers were measured via CCK8 assay. Points are 450 Mean \pm SD of 12 wells normalized to Day $0 . * *: p<0.01$

451 c-d. Inactivation of HRAS or NRAS increases H23 colony formation. Wildtype (sgSAFE), HRAS452 knockout $(\operatorname{sg} H R A S)$, or NRAS-knockout $(\operatorname{sg} N R A S)$ H23 cells were seeded at 1000 cells/well in 6453 well plates and grown for two weeks. Cells were stained with crystal violet. c.

454 Representative images. Scale bar $=5 \mathrm{~mm}$. d. Mean \pm SD of colony number of 12 fields. $* *: p<0.01$ 455 e-f. Re-expression of wild type HRAS suppresses HRAS-null H23 cell colony formation. TRE456 Ctrl or TRE-HRAS H23 cells were seeded at 1000 cells/well in 6-well plates and grown with or 457 without $50 \mathrm{ng} / \mathrm{ml}$ Dox for two weeks. Cells were stained with crystal violet. e. Representative 458 images. Scale bar $=5 \mathrm{~mm}$. f. Mean \pm SD of colony number of 12 fields. $* *: p<0.01$

459 g-k. Inactivation of wild type HRAS or NRAS increases H23 cell growth after transplantation. g. 460 Schematic of tumor initiation with subcutaneous (SubQ) or intravenous (IV) transplantation of 
461 H23 cells with inactivation of HRAS or NRAS in NSG mice. Mouse number, cell number, and

462 tumor growth time after transplantation are indicated. $\mathbf{h}$. Tumor weight from SubQ transplantation

463 of indicated cells. Each dot represents a mouse. Mean value was shown. i. Ki67 ${ }^{\text {pos }}$ cell number in

464 tumor section from SubQ transplantation of indicated cells was shown as Mean \pm SD value of 20

465 view fields. j. Tumor area (percentage of h-mitochondriapos area) from IV transplantation of

466 indicated cells. Each dot represents a tumor. Mean value was shown. k. Ki67 ${ }^{\text {pos }}$ cell number in

467 tumor section from IV transplantation of indicated cells is shown as Mean \pm SD value of 20 view

468 fields $(200 \mathrm{x}) . *$ : $\mathrm{p}<0.05 ; * *: \mathrm{p}<0.01$; ns: not significant.

469

470 Figure 4. Wildtype RAS paralogs suppress RAS signaling

471 a. Representative image of pERK staining in $K T ; H 11^{L S L-C a s 9}$ mice with tumors initiated with Lenti-

472 sgRNA/Cre vectors as indicated. Quantification of $\mathrm{pERK}^{\mathrm{pos}}$ cells per tumor was shown as

473 Mean \pm SD of 20 tumors. *: $\mathrm{p}<0.05 ; * *: \mathrm{p}<0.01$; Scale bar: $100 \mu \mathrm{m}$

474 b. Representative image of pERK staining in subcutaneous tumor transplanted with $\mathrm{H} 23$ cells as

475 indicated. Quantification of $\mathrm{pERK}^{\text {pos }}$ cells per field was shown as Mean $\pm \mathrm{SD}$ of 20 fields. **:

$476 \mathrm{p}<0.01$; Scale bar: $100 \mu \mathrm{m}$. HSP90 shows loading.

477 c. Western blot analysis of sorted cancer cells from $K T ; H 11^{L S L-C a s 9}$ mice transduced with Lenti-

478 sgRNA/Cre vectors as indicated. Multiple tumors were pooled and Tomato ${ }^{\text {pos }}$ cancer cells were

479 sorted prior to and protein extraction. HSP90 shows loading.

480 d. Western blot analysis of murine lung adenocarcinoma cell line that was transduced with Lenti-

481 sgRNA vectors as indicated and selected with puromycin to generate stable knockout cell lines.

482 Wildtype cells (sgNeo) or HRAS- or NRAS-knockout cells (sgHras, sgNras) were cultured under

483 limited serum (1\%) for 2 days before protein extraction. HSP90 shows loading. 
484 e. Western blot analysis of cultured human lung adenocarcinoma cell lines transduced with Lenti485 sgRNA vectors as indicated and selected with puromycin to generate stable knockout cell lines. 486 Wildtype cell (sgSAFE) or HRAS- or NRAS-knockout cells ( $\operatorname{sg} H R A S, \operatorname{sg} N R A S)$ were 487 cultured under limited serum (1\%) for 2 days before protein extraction. HSP90 shows loading.

488 f. Western blot analysis of human lung adenocarcinoma cell lines re-expression HRAS (TRE489 HRAS) under Doxycycline (Dox) treatment. HRAS-null cells were generated as described in 490 Figure 3a. HRAS-null cells were re-transduced with lentiviral vector expressing TRE-HRAS at 491 high MOI (>5) to generate stable HRAS re-expression cells (sgHRAS-TRE-HRAS). To re-express 492 HRAS, cells were treated with 0,1 , or $2 \mathrm{ng} / \mathrm{ml}$ Dox and cultured under limited serum (1\%) for 2 493 days before protein extraction. HSP90 shows loading.

494 g. Comparison of GI50 values to MEK inhibitors trametinib among wildtype and HRAS-null H23 495 cells under treatment of indicated dose of trametinib for four days. Cell numbers were measured 496 via CCK8 assay and normalized to cells treated with vehicle. Each data point was 497 shown as Mean \pm SD of 12 wells.

498 h. Comparison of GI50 values to MEK inhibitors trametinib among HRAS-null H23 cells (H23499 sgHRAS) re-expressing HRAS in presence (HRAS+Dox) or absence (HRAS) of Doxycycline plus 500 indicated dose of trametinib for four days. Cell numbers were measured via CCK8 assay and 501 normalized to cells treated with vehicle. Each data point was shown as Mean \pm SD of 12 wells.

503 Figure 5. Wildtype RAS paralogs fine-tune RAS signaling through interaction with 504 oncogenic KRAS.

505 a. Bubble plot of three AP/MS experiments with H-, K-, and N-RAS as baits (rows), showing the 506 enrichment of their paralogs (columns). 
507 b. Diagram of the ReBiL2.0 system. KRAS ${ }^{\mathrm{G} 12 \mathrm{D}}-\mathrm{KRAS}^{\mathrm{G} 12 \mathrm{D}}$ interactions were quantified by 508 normalized luminescent signal generated by membrane association facilitated interaction of the 509 split-luciferase that is fused to the N-terminus of $\mathrm{KRAS}^{\mathrm{G} 12 \mathrm{D}}$ (upper). Split-luciferase that is fused 510 to the last four amino acids of KRAS (CVIM) is applied as control for background split-luciferase 511 interaction on the membrane (lower). Adapted from Li et al. 2020.

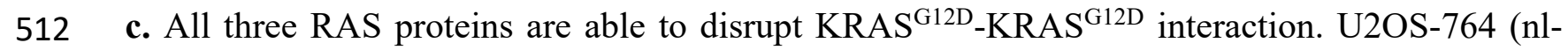

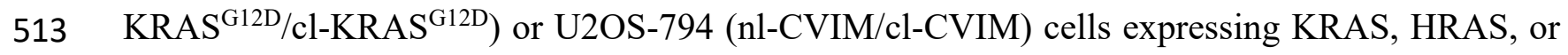
514 NRAS were cultured in limited serum (1\%) under $100 \mathrm{ng} / \mathrm{ml}$ Doxycycline (Dox) for 24 hours. 515 ReBiL2.0 assay were performed as previously described and detailed in Methods. Points are 516 Mean \pm SD ReBiL2.0 score of 36 wells normalized to cells transduced with empty lentiviral vector. $517 * *: \mathrm{p}<0.01$

518 d. Pan-cancer frequency of HRAS mutations in patients with wildtype and oncogenic KRAS519 tumors from Project GENIE. Known oncogenic HRAS mutations are highlighted in red. The 520 dashed line indicates equal mutation frequency in KRAS-wildtype and mutant samples. Four 521 candidate mutations that were chosen for further validation in this study were highlighted.

522 e. HRAS $^{\mathrm{T} 50 \mathrm{M}}$ and HRAS $^{\mathrm{R} 123 \mathrm{C}}$ are novel RAS-RAS interaction deficient mutations. U2OS-764 (nl-

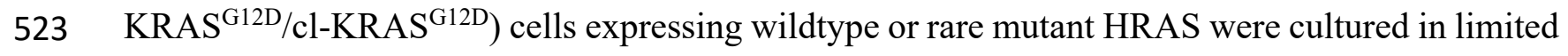
524 serum (1\%) under $100 \mathrm{ng} / \mathrm{ml}$ Dox for 24 hours. Points are Mean \pm SD ReBiL2.0 score of 12 wells 525 normalized to cells transduced with empty lentiviral vector (upper). ${ }^{* *}$ : $p<0.01$; ns: not significant. 526 HRAS (wildtype and mutant) protein expression level in corresponding cells were shown by 527 Western blot analysis (lower). 
528 f. $\operatorname{HRAS}^{\mathrm{T} 50 \mathrm{M}}$ and $\operatorname{HRAS}^{\mathrm{R} 123 \mathrm{C}}$ are located close to the predicted HRAS-KRAS interaction interface.

529 HRAS is shown in light orange and $\mathrm{KRAS}^{\mathrm{G} 12 \mathrm{D}}$ is shown in blue. Residue R123 (in magenta) makes

530 an intrachain salt bridge with E143 (in cyan).

531 g. Prey RAF proteins enriched in each experiment with the indicated baits in A549 cells (for K-,

532 H-, or N-RAS) or HEK293 cells (for KRAS). Yellow color indicates higher values of NSAF. Both

533 GTP- and GDP-bond HRAS behave like GDP-bond KRAS in their RAF interactions.

534 h. Western blot analysis of cultured HRAS-null HOP62 cells (HOP62-Cas9-sgHRAS) re-

535 expressing wildtype or mutants (T50M, Y64A, or R123C) under Dox treatment. Cells were

536 cultured under limited serum (1\%) for 2 days before protein extraction. Re-expression of HRAS

537 mutations have no effects on ERK phosphorylation.

538 i. Cell proliferation of cultured HRAS-null HOP62 cells (HOP62-Cas9-sgHRAS) re-expressing 539 wildtype or mutants (T50M, Y64A, or R123C) under Dox treatment. Cells were cultured in limited 540 serum (1\%) with or without Dox for 4 days. Cell viability was measured via CCK8 assay and 541 normalized to cells treated with vehicle. Re-expression of HRAS mutants have no effects on cell 542 proliferation.

543

544 Figure 6. Paired screens in KRAS-driven and BRAF-driven lung cancer models validates 545 HRAS and NRAS as KRAS-specific tumor suppressors.

546 a-b. Schematic of pairwise screen of tumor suppressive function in KRAS- and BRAF-driven lung 547 cancer. A pool of barcoded Lenti-sgRNA/Cre vectors targeting top mediators of KRAS-driven 548 lung tumor growth (Lenti-sgMultiGEMM/Cre) was used to initiate tumors in cohorts of $549 \mathrm{KT} ; H 11^{L S L-C a s 9 /++}$ and Braf ${ }^{C A /+} T ; H 11^{L S L-C a s 9 /+}\left(B r a f T ; H 11^{L S L-C a s 9 /++}\right)$ mice. Each regulator of KRAS550 driven tumor growth (Hras, Nras, Nme2 and Fnta) was targeted by two sgRNAs (those with the 
551 largest effect size in the validation screen). The pool also included four Inert sgRNAs, as well as

552 sgRNAs targeting $A p c, C d k n 2 a$, and $R b m 10$ as tumor suppressor controls (a). Tumors were

553 initiated through intratracheal delivery of Lenti-sgMultiGEMM/Cre, and Tuba-seq was performed

554 on each tumor-bearing lung 15 weeks after initiation, followed by analysis of sgID-BC sequencing

555 data to characterize the effects of inactivating each gene (b).

556 c. Fluorescence images of representative lung lobes 15 weeks after tumor initiation. Scale bars $=$ $5575 \mathrm{~mm}$. Lung lobes are outlined with a white dashed line.

558 d. Total lung weight in KT;H1 $1^{\text {LSL-Cas9/+ }}$ and BrafT;H1 $1^{\text {LSL-Cas } 9 /+}$ mice 15 weeks after tumor 559 initiation. Each dot is a mouse and mean value is indicated. **: $\mathrm{p}<0.01$

560 e-f. Size distribution of sgInert tumors in $K T ; H 11^{L S L-C a s 9 /+}$ and BrafT;H1 $1^{L S L-C a s 9 /+}$ mice. In e., each

561 dot represents a tumor, and the area of each dot is proportional to the number of cancer cells in 562 that tumor. To prevent overplotting a random sample of 1,000 tumors from each of five 563 representative $K T ; H 11^{L S L-C a s 9 /+}$ and BrafT;H1 $1^{L S L-C a s 9 /+}$ mice are plotted. In f., the empirical 564 cumulative distribution function of tumor sizes across all KT;H1 $1^{L S L-C a s 9 /+}$ and BrafT;H1 $1^{L S L-C a s 9 /+}$ 565 mice are plotted. Tumors $>500$ cells in size are shown.

566 g. Inactivation of Hras and Nras increases tumor size in KT;H1 $1^{\text {LSL-Cas9/+ }}$ but not BrafT;H1 $1^{L S L-}$ 567 Cas $9 /+$ models. Tumor sizes at indicated percentiles for each sgRNA relative to the size of sgInert568 containing tumors at the corresponding percentiles in $K T ; H 11^{L S L-C a s 9 /+}$ (left, white background) 569 and BrafT;H11 $1^{\text {LSL-Cas } 9 /+}$ (right, gray background) mice. Line at $\mathrm{y}=1$ indicates no effect relative to 570 sgInert. Error bars indicate 95\% confidence intervals. Percentiles that are significantly different 571 from sgInert (two-sided FDR-adjusted $\mathrm{p}<0.05$ ) are in color. Confidence intervals and P-values 572 were calculated by bootstrap resampling. 
573 h. Comparison of the effects of inactivation of known tumor suppressors (Rbm10, Apc, and $574 C d k n 2 a)$ on tumor size in KT;H11 $1^{L S L-C a s 9 /+}$ and BrafT;H11 $1^{L S L-C a s 9 /+}$ models. Tumor sizes at 575 indicated percentiles for each sgRNA relative to the size of sgInert-containing tumors at the 576 corresponding percentiles in KT;H11 $1^{\text {LSL-Cas9/+ }}$ (left, white background) and BrafT;H1 $1^{\text {LSL-Cas9/+ }}$

577 (right, gray background) mice. Line at $\mathrm{y}=1$ indicates no effect relative to sgInert. Error bars indicate $57895 \%$ confidence intervals. Percentiles that are significantly different from sgInert (two-sided FDR579 adjusted $\mathrm{p}<0.05)$ are in color. Confidence intervals and P-values were calculated by bootstrap 580 resampling.

581 i. Wildtype RAS paralogs function as tumor suppressors in oncogenic KRAS-driven lung cancer.

582 Left panel, in oncogenic KRAS-driven lung cancer cells, wildtype RAS paralogs competitively 583 interact with oncogenic KRAS and suppress oncogenic KRAS clustering. Right panel, inactivation 584 of wildtype RAS allele, or "RAS paralog imbalance", hyper-activate oncogenic KRAS signaling 585 and promotes lung cancer growth.

586

587

588

589

590

591

592

593

594

595 


\section{Supplemental Figure 1. Prioritize candidate KRAS-interacting proteins for this study.}

597 a. Flow chart for prioritizing candidate KRAS-interacting proteins for this study. Candidate 598 KRAS-interacting proteins were chosen based on multiple criteria including their interaction with 599 KRAS, their homolog mRNA expression in $\mathrm{Kras}^{\mathrm{G} 12 \mathrm{D}}$-driven lung cancer in mouse model, and the 600 consistency for them to bind different RAS-GTPase. RADIL is added at the last step due to its 601 validated importance in KRAS-mutant human cell lines.

602 b. Candidate proteins interact with KRAS from two protein-protein interaction analyses (Kelly, 603 Kostyrko, Han et al. 2020; Broyde, Simpson, Murray et al. 2020). Shared KRAS-interaction 604 proteins are shown as their log10NSAF and SigMap Score.

605 c. Homolog mRNA expression (TPM) of candidate KRAS-interacting proteins in $\mathrm{Kras}^{\mathrm{G} 12 \mathrm{D}}$-driven 606 lung cancer in mouse model (Chuang et al. 2017).

607 d. Bubble plot of eight AP/MS experiments with GTP- and GDP-locked mutant GTPases as baits 608 (rows), showing the enrichment of selected candidate KRAS-interacting proteins (columns). Dark 609 borders indicate FDR $<0.05$.

610 e. Mutation frequencies of these 13 candidate genes in lung adenocarcinoma (data from TCGA, 611 Nat. Genet. 2016).

613 Supplemental Figure 2. Tumor barcoding coupled with barcode sequencing (Tuba-seq) can uncover engineered alterations that reduce tumor number and growth.

615 a-b. Schematic of the Tuba-seq approach to measure the effects of essential gene inactivation on 616 tumor growth. Lentiviral-sgRNA/Cre vectors with inert sgRNAs (gray) or sgRNAs targeting 617 known essential genes (navy) were diversified with a two component sgID-BC. A vector targeting 618 known tumor suppressor $A p c$ was included as a positive control (a). Tumors were initiated with 
619 this barcoded Lenti-sgEssential/Cre pool in $K T$ and $K T ; H 11^{L S L-C a s 9}$ mice. Tuba-seq was performed 620 on each tumor-bearing lung 12 weeks after initiation, followed by analyses of sgID-BC sequencing 621 data to characterize the effects of inactivating each gene (b).

622 c. Tumor sizes at indicated percentiles for each sgRNA relative to the size of sgInert-containing 623 tumors at the corresponding percentiles. Line at $y=1$ indicates no effect relative to $\operatorname{sgInert.~Error~}$ 624 bars indicate $95 \%$ confidence intervals. Percentiles that are significantly different from sgInert 625 (two-sided FDR-corrected $\mathrm{p}<0.05$ ) are in color. Confidence intervals and P-values were 626 calculated by bootstrap resampling.

627 d. The impact of each sgRNA on mean tumor size relative to sgInerts, assuming a log-normal 628 distribution of tumor sizes (LNmean). sgRNAs with two-sided $\mathrm{P}<0.05$ after FDR-adjustment are 629 in bold.

630 e. The impact of each sgRNA on tumor burden (number of neoplastic cells aggregated across all 631 tumors of a genotype) relative to sgInerts and normalized to the same statistic in $K T$ mice to 632 account for representation of each sgRNA in the viral pool. sgInerts are in gray and the line at $\mathrm{y}=1$ 633 indicates no effect. Error bars indicate 95\% confidence intervals. Relative burdens significantly 634 different from sgInert (two-sided FDR-corrected $\mathrm{p}<0.05$ ) are in color. Confidence intervals and P635 values were calculated by bootstrap resampling.

636 f. The impact of each sgRNA on tumor number relative to sgInerts and normalized to the same 637 statistic in $K T$ mice to account for representation of each sgRNA in the viral pool. sgInerts are in 638 gray and the line at $y=1$ indicates no effect. Error bars indicate $95 \%$ confidence intervals. Relative 639 tumor numbers significantly different from sgInert (two-sided FDR-corrected $\mathrm{p}<0.05$ ) are in color. 640 Confidence intervals and P-values were calculated by bootstrap resampling. 
641 g. The impact of each sgRNA on tumor number plotted against its impact on LNmean tumor size.

642 The lines at $\mathrm{y}=1$ and $\mathrm{x}=1$ indicate no effect relative to sgInert on tumor number and size,

643 respectively. $\operatorname{sgRsp19}$ and sgPcna cluster in the lower left quadrant near $\mathrm{x}=1$, indicating that

644 targeting essential genes strongly reduces tumor number but only moderately decreases average

645 tumor size. Error bars indicate 95\% confidence intervals calculated by bootstrap resampling.

646

647 Supplemental Figure 3. Inactivation of KRAS-interacting proteins has similar impacts on

648 tumor growth in p53-proficient and p53-deficient contexts.

649 a. Tumor sizes at indicated percentiles for each sgRNA relative to the size of sgInert-containing

650 tumors at the corresponding percentiles in $K T$ mice. $K T$ mice lack Cas9, thus all sgRNAs are

651 functionally equivalent to sgInerts. Genes are ordered as in Figure 1f. Line at $y=1$ indicates no

652 effect relative to sgInert. Error bars indicate 95\% confidence intervals. Confidence intervals and

653 P-values were calculated by bootstrap resampling. As expected, no percentiles were significantly

654 different from sgInert (two-sided FDR-adjusted $\mathrm{p}<0.05$ ).

655 b. The impact of each sgRNA on mean tumor size relative to $\operatorname{sgInerts}$ in $K T ; H 11^{L S L-C a s 9}$, assuming

656 a log-normal distribution of tumor sizes (LNmean). sgRNAs with two-sided $\mathrm{P}<0.05$ after FDR-

657 adjustment are in bold. P-values were calculated by bootstrap resampling.

658 c-d. The impact of each sgRNA on tumor burden (c) and number (d) relative to sgInerts in $659 K T ; H 11^{L S L-C a s 9}$ mice, normalized to the corresponding statistic in $K T$ mice to account for 660 representation of each sgRNA in the viral pool. sgInerts are in gray and the line at $\mathrm{y}=1$ indicates 661 no effect. Error bars indicate 95\% confidence intervals. Relative tumor burdens and numbers 662 significantly different from sgInert (two-sided FDR-adjusted $\mathrm{p}<0.05$ ) are in color. Confidence 663 intervals and P-values were calculated by bootstrap resampling. 
664 e. Tumor sizes at the indicated percentiles for each sgRNA relative to the size of sgInert-containing 665 tumors in KT; $553^{\text {floxflox }} ; H 11^{\text {LSL-Cas } 9}$ mice. Genes are ordered as in Figure 1f. Dashed line indicates 666 no effect relative to sgInert. Error bars indicate $95 \%$ confidence intervals. Percentiles that are 667 significantly different from sgInert (two-sided FDR-adjusted $p<0.05$ ) are in color. Confidence 668 intervals and P-values calculated by bootstrap resampling.

669 f-h. Comparison of the impact of each sgRNA on relative LNmean tumor size (f), tumor burden 670 (g) and tumor number (h) in KT;H1 $1^{\text {LSL-Cas9 }}$ and $K T ; p 53^{\text {floxflox }} ; H 11^{\text {LSL-Cas9 }}$ mice. Error bars indicate $67195 \%$ confidence intervals calculated by bootstrap resampling.

672

673 Supplemental Figure 4. Top candidate KRAS-interacting proteins from initial Tuba-seq 674 screen impact multiple metrics of tumor growth in validation cohort.

675 a. Tumor sizes at indicated percentiles for each sgRNA relative to the size of sgInert-containing 676 tumors at the corresponding percentiles in $K T$ mice. $K T$ mice lack Cas9, thus all $\operatorname{sgRNAs}$ are 677 functionally equivalent to sgInerts. Genes are ordered as in Figure 2d, but note the change in axis 678 scaling. Line at $y=1$ indicates no effect relative to sgInerts. Error bars indicate $95 \%$ confidence 679 intervals. Confidence intervals and P-values were calculated by bootstrap resampling. As expected, 680 no percentiles were significantly different from sgInert (FDR-adjusted $\mathrm{p}<0.05)$.

681 b. The impact of each sgRNA on mean tumor size relative to sgInerts, assuming a log-normal 682 distribution of tumor sizes (LNmean). Two-sided P-values were calculated by bootstrap 683 resampling. sgRNAs with $\mathrm{P}<0.05$ after FDR-adjustment are in bold. Note that this data for the 684 sgInerts, sgHras\#1-3 and sgNras\#1-3 is also plotted in Figure 2e. 
685 c. The impact of each sgRNA on tumor burden relative to sgInerts in $K T ; H 11^{L S L \text {-Cas } 9}$ mice, 686 normalized to the corresponding statistic in $K T$ mice to account for representation of each sgRNA

687 in the viral pool. sgInerts are in gray and the line at $\mathrm{y}=1$ indicates no effect. Error bars

688 indicate $95 \%$ confidence intervals. Relative tumor burdens significantly different from sgInert 689 (two-sided FDR-adjusted $\mathrm{p}<0.05$ ) are in color. Confidence intervals and P-values were calculated 690 by bootstrap resampling.

691 d. The impact of each sgRNA on tumor number relative to sgInerts in $K T ; H 11^{L S L \text {-Cas } 9}$ mice, 692 normalized to the corresponding statistic in $K T$ mice to account for representation of each sgRNA 693 in the viral pool. sgInerts are in gray and the line at $y=1$ indicates no effect. Error bars

694 indicate 95\% confidence intervals. Relative tumor numbers significantly different from sgInert 695 (two-sided FDR-adjusted $\mathrm{p}<0.05)$ are in color. Confidence intervals and P-values were calculated 696 by bootstrap resampling.

697

698 Supplemental Figure 5. Dependency of human LUAD cell lines on RAS family members.

699 a. Comparison of RAS family member dependency scores between KRAS mutant and KRAS 700 wildtype human LUAD cell lines. $* * * *(p<0.0001)$, ns (not-significant).

701 b. Volcano plot showing the effects of RAS gene knockouts in A549 cells. The T-score represents 702 the normalized effect of multiple sgRNAs targeting a gene. A positive T-score indicates a tumor 703 suppressive effect. The effects of each gene relative to SAFE sgRNAs were tested via Mann704 Whitney U (MWU) test, corrected via Benjamini-Hochberg procedure and shown as $705 \log 10($ MWU-Adjusted P-val). (Data source: Kelly, Kostyrko, Han et al. 2020)

706 c. Volcano plot showing effects of RAS gene knockouts in KRAS-mutant human LUAD cells 707 (left: H2009, mid: H23, right: H1975) in 3D culture. The T-score represents the normalized effect 
708 of multiple sgRNAs targeting a gene. A positive T-score indicates a tumor suppressive effect. The

709 effects of each gene relative to SAFE sgRNAs were tested via two-side t-test, corrected via

710 Benjamini-Hochberg procedure and shown as -log10(Q-val). (Data source: Han et al. 2020)

711

712 Supplemental Figure 6. Inactivation of wild type HRAS or NRAS increases H23 cell growth

713 after transplantation.

714 a. Representative image of subcutaneous tumor size four weeks after transplantation with $\mathrm{H} 23$

715 cells as indicated. Quantification was shown in Figure 3h. Scale bar: $2 \mathrm{~mm}$

716 b. Representative image of Ki67 staining from subcutaneous tumor four weeks after

717 transplantation with H23 cells as indicated. Quantification was shown in Figure 3i. Scale bar: 100

$718 \mu \mathrm{m}$

719 c. Representative image of HE (upper) and human mitochondria (lower) staining from lung tumor

720 four weeks after intravenous transplantation with $\mathrm{H} 23$ cells as indicated. Quantification was shown

721 in Figure 3j. Scale bar: $500 \mu \mathrm{m}$

722 d. Representative image of Ki67 staining from lung tumor four weeks after intravenous

723 transplantation with H23 cells as indicated. Quantification was shown in Figure 3k. Scale bar: 200

$724 \mu \mathrm{m}$

725

726 Supplemental Figure 7. Wildtype RAS paralogs finetune RAS signaling.

727 a. Quantification of $\mathrm{pERK}^{\mathrm{pos}}$ cells in $K T ; H 11^{L S L-C a s 9}$ mice with tumors initiated with Lenti-

728 sgRNA/Cre vectors as indicated in Figure 4a. Each dot represents a tumor. *: $\mathrm{p}<0.05 ;{ }^{* *}$ : $\mathrm{p}<0.01$

729 b. Quantification of $p E R K^{\text {pos }}$ cells per field of indicated cells from Figure $4 \mathbf{b}$. Each dot represents

730 a view field. $* *$ : $p<0.01$ 
731 c-f. Raw images for western blots from Figure 4c-f. HRAS expression on Figure 4f were detected

732 using same lysis on a different gel with increased loading.

733

734 Supplemental Figure 8. Identification of rare HRAS mutations in oncogenic KRAS-mutant 735 tumors.

736 a. Pan-cancer frequency of $H R A S$ mutations in patients with $K R A S$-wildtype and oncogenic $K R A S$ -

737 mutant tumors from Project GENIE. Mutations that are intergenic, intronic, silent, or fall in the 3'

738 or 5' UTR were excluded. Oncogenic KRAS mutants were defined as tumors having missense

739 mutations in codons 12,13 or 61 . Known oncogenic $H R A S$ mutations are highlighted in red. The

740 dashed line indicates equal mutation frequency in $K R A S$-wildtype and mutant samples. Non-

741 oncogenic mutations occurring at least once in patients with oncogenic $K R A S$ mutations are

742 annotated. HRAS mutants selected for analysis of ability to disrupt KRAS ${ }^{\mathrm{G} 12 \mathrm{D}}$-KRAS ${ }^{\mathrm{G} 12 \mathrm{D}}$

743 interactions are highlighted in bold.

744 b. Characteristic of samples with rare HRAS mutants selected for analysis of their ability to disrupt 745 KRAS $^{\mathrm{G} 12 \mathrm{D}}$-KRAS ${ }^{\mathrm{G} 12 \mathrm{D}}$ interactions using the ReBiL2.0 system.

746

747 Supplemental Figure 9. Modeling RAS-RAS dimer.

748 a. Homodimers of RAS present in crystals of HRAS, KRAS, and NRAS in the Protein Data Bank.

749 Dimers were downloaded from the Protein Common Interface Database (ProtCID) ${ }^{58}$, which

750 clusters interfaces present in different crystals of homologous proteins. The $\alpha 4-\alpha 5$ dimer shown

751 is present in 84 entries of HRAS, 13 entries of KRAS, and one entry of NRAS (PDB 5UHV).

752 b. Models of a homodimer of $\mathrm{KRAS}^{\mathrm{G} 12 \mathrm{D}}$ and heterodimers of KRAS ${ }^{\mathrm{G} 12 \mathrm{D}}$ with HRAS, HRAS ${ }^{\mathrm{T} 50 \mathrm{M}}$,

753 and HRAS ${ }^{\mathrm{R} 123 \mathrm{C}}$. The $\alpha^{4-\alpha}$. HRAS dimer from PDB entry $3 \mathrm{~K} 8 \mathrm{Y}$ was used as a template. 
754 KRAS $^{\mathrm{G} 12 \mathrm{D}}$ from PDB entry 5USJ was superposed with the program PyMol on one or both 755 monomers of $3 \mathrm{~K} 8 \mathrm{Y}$ to form the heterodimers and the homodimer respectively. Residues T50 and 756 R123 were mutated with PyMol. All four structures were relaxed with the program Rosetta using 757 the FastRelax protocol with the Ref2015 scoring function) ${ }^{59}$. Rosetta uses the backbone-dependent 758 rotamer library of Shapovalov and Dunbrack to repack side chains around the mutated sites ${ }^{60}$. The 759 resulting energies were: $\mathrm{KRAS}^{\mathrm{G} 12 \mathrm{D}}$-KRAS ${ }^{\mathrm{G} 12 \mathrm{D}},-1122.8 \mathrm{kcal} / \mathrm{mol}$; HRAS-KRAS ${ }^{\mathrm{G} 12 \mathrm{D}},-1144.8$

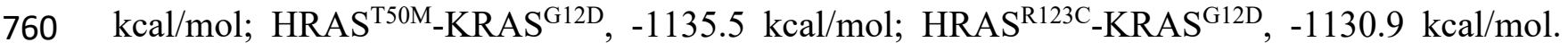
761 Residues T50 (magenta) and R123 (orange) are indicated in sticks.

762

763 Supplemental Figure 10. Wildtype RAS paralogs finetune RAS signaling through interaction 764 with oncogenic KRAS.

765 a. Raw images for western blots of split-luciferase (HA-tag) expression for ReBiL2.0 from Figure 766 5c. HA-tag expression were detected using same lysis on a different gel with increased loading.

767 b. Raw images for western blots of split-luciferase (HA-tag) expression for ReBiL2.0 from Figure 768 5e. HA-tag expression were detected using same lysis on a different gel with increased loading. 769 c. Raw images for western blots from Figure 5h. HRAS expression were detected using same lysis 770 on a different gel with increased loading.

771

772 Supplemental Figure 11. Paired screen in KRAS-driven and BRAF-driven lung cancer 773 models validates HRAS and NRAS as KRAS-specific tumor suppressors.

774 a-c. Tumor sizes at indicated percentiles for each sgRNA relative to the size of sgInert-containing 775 tumors at the corresponding percentiles in KT;H11 $1^{\text {LSL-Cas9/+ }}$ (a), BrafT;H1 $1^{\text {LSL-Cas9/+ }}$ (b) and KT 776 mice $(\mathbf{c})$. Genes are ordered by $95^{\text {th }}$ percentile tumor size in $K T ; H 11^{L L L-C a s 9 /+}$ mice, with sgInerts 
777 on the left. sgInerts are in gray, and line at $\mathrm{y}=1$ indicates no effect relative to sgInert. Error bars

778 indicate $95 \%$ confidence intervals. Percentiles that are significantly different from sgInert (two-

779 sided FDR-adjusted $\mathrm{p}<0.05$ ) are in color. Confidence intervals and P-values were calculated by 780 bootstrap resampling. The negative effects of sgRNAs targeting Fnta and Nme2 in the KT mice

781 (c) are unexpected and indicate a potential bias in the size distributions of tumors with these 782 genotypes. We note that the same bias may be present in the KT;H1 $1^{L S L-C a s 9 /+}$ and BrafT;H1 $1^{L S L-}$

$783 \mathrm{Cas} 9 /+$ data; however, sgRNAs targeting these genes in previous experiments showed consistent 784 negative effects on tumor size, suggesting that the observed effects in this $K T ; H 11^{\text {LSL-Cas9/+ }}$ cohort 785 are not solely the product of this bias.

786 d. The impact of each sgRNA on tumor burden relative to sgInerts in KT;H1 $1^{\text {LSLCas } 9 /+}$ (top) and 787 BrafT;H1 $1^{L S L-C a s 9 /+}$ (bottom) mice, normalized to the corresponding statistic in KT mice to account 788 for representation of each sgRNA in the viral pool. sgInerts are in gray and the line at $789 \mathrm{y}=1$ indicates no effect. Error bars indicate $95 \%$ confidence intervals. Relative tumor burdens 790 significantly different from sgInert (two-sided FDR-adjusted $\mathrm{p}<0.05$ ) are in color. Confidence 791 intervals and P-values were calculated by bootstrap resampling.

792 e. The impact of each sgRNA on tumor number relative to sgInerts in $K T ; H 11^{L S L-C a s 9 /+}$ (top) and 793 BrafT;H11 $1^{L S L-C a s 9 /+}$ (bottom) mice, normalized to the corresponding statistic in KT mice to account 794 for representation of each sgRNA in the viral pool. sgInerts are in gray and the line at $795 \mathrm{y}=1$ indicates no effect. Error bars indicate $95 \%$ confidence intervals. Relative tumor numbers 796 significantly different from sgInert (two-sided FDR-adjusted $\mathrm{p}<0.05$ ) are in color. Confidence 797 intervals and P-values were calculated by bootstrap resampling. 


\section{METHODS}

801 Cells, Reagents and Plasmids:

802 H23, H727, and HOP62 cells were originally purchased from ATCC; HC494(KPT) lung 803 adenocarcinoma cells were generated in the Winslow Lab; U2OS-134-764np (nl-KRAS ${ }^{\mathrm{G} 12 \mathrm{D}}$ cl-

804 KRAS $^{\text {G12D }}$; KRAS ${ }^{\text {G12D }}$ was fused to the N-termini of split luciferase proteins) and U2OS-134-794p 805 (nl-CVIM cl-CVIM; CVIM represents the C-terminal last 20 amino acids of KRAS4B) cells were 806 generated in the Wahl lab by Dr. Yao-Cheng Li (Salk Institute for Biological Studies). HC494 807 cells were cultured in DMEM containing 10\% FBS, 100 units $/ \mathrm{mL}$ penicillin and $100 \mu \mathrm{g} / \mathrm{mL}$ 808 streptomycin. A549, H460 and H82 cells were cultured in RPMI1640 media containing 10\% FBS, 809100 units/mL penicillin and $100 \mu \mathrm{g} / \mathrm{mL}$ streptomycin. U2OS cells were cultured in DMEM/F12 810 (Thermo Fisher; phenol-red free), 10\% (vol/vol) FBS, and $10 \mu \mathrm{g} / \mathrm{mL}$ ciprofloxacin. All cell lines 811 were confirmed to be mycoplasma negative (MycoAlert Detection Kit, Lonza).

812 Trametinib was purchased from MedChemExpress (HY-10999); 5-Bromo-2'-deoxyuridine 813 (10280879001) and D-Luciferin (L9504-5MG) was purchased from Sigma-Aldrich. All plasmids 814 used in this study were listed in supplementary Table 1 and will be donated to Addgene.

816 Design, generation, barcoding, and production of lentiviral vectors

817 The sgRNA sequences targeting the putative tumor suppressor genes were designed using 818 CRISPick (https://portals.broadinstitute.org/gppx/crispick/public). All sgRNA sequence are 819 shown in Supplementary Table 2. Each desired sgRNA vector was modified from our previously 820 published pll3-U6-sgRNA-Pgk-Cre vector via site-directed mutagenesis (New England Biolabs, 821 E0554S). The generation of the barcode fragment containing the 8-nucleotide sgID sequence and 
822 20-nucleotide degenerate barcode, and subsequent ligation into the vectors were performed as 823 previously described.

824 Lentiviral vectors were produced using polyethylenimine (PEI)-based transfection of 293T cells 825 with delta8.2 and VSV-G packaging plasmids in 150-mm cell culture plates. Sodium butyrate 826 (Sigma Aldrich, B5887) was added 8 hours after transfection to achieve a final concentration of

$82720 \mathrm{mM}$. Medium was refreshed 24 hours after transfection. $20 \mathrm{~mL}$ of virus-containing supernatant 828 was collected 36,48 , and 60 hours after transfection. The three collections were then pooled and 829 concentrated by ultracentrifugation ( $25,000 \mathrm{rpm}$ for 1.5 hours), resuspended overnight in $100 \mu \mathrm{L}$ 830 PBS, then frozen at $-80^{\circ} \mathrm{C}$ and were thawed and pooled at equal ratios immediately prior to delivery 831 to mice.

832

833 Mice and tumor initiation

834 The use of mice for the current study has been approved by Institutional Animal Care and 835 Use Committee at Stanford University, protocol number 26696.

$836 \mathrm{Kras}^{\mathrm{LSL}-\mathrm{G} 12 \mathrm{D} /+}$ (RRID:IMSR_JAX:008179), R26 ${ }^{\text {LSL-tdTomato }}$ (RRID:IMSR_JAX:007909),

837 and H11 $1^{\text {LSL-Cas9 }}$ (RRID:IMSR_JAX:027632) mice have been previously described. They were on 838 a C57BL/6:129 mixed background. The B6.129P2(Cg)-Braf ${ }^{\mathrm{fm} 1 \mathrm{Mmcm} / \mathrm{J}}\left(\mathrm{BRAF}^{\mathrm{F}-\mathrm{V} 600 \mathrm{E}}\right)$ mice 839 were initially generated by Dankort et al. and obtained from the Jackson Laboratory 840 (RRID:IMSR_JAX: 017837). Tumors were initiated by intratracheal delivery of $60 \mu \mathrm{l}$ of

841 lentiviral vectors dissolved in PBS.

842 For the initial experiments in Figure 1 and 2, tumors were allowed to develop for 12 weeks after 843 viral delivery of a lentiviral pool that contained 19 barcoded Lenti-sgRNA/Cre vectors (Lenti- 
$844 \operatorname{sgKrasIP/Cre)}$. Tumors were initiated in $\operatorname{Kras}^{\text {LSL-G12D; R26 }}{ }^{\text {LSL-tdTomato }}(K T), K T ; \mathrm{H} 11^{\text {LSL-Cas9; }}$ or $845 K T ; p 53^{f l f l} ; \mathrm{H} 11^{\text {LSL-Cas9 }}$ mice with $1.95 \times 10^{5}$ infectious units (ifu)/mouse.

846 For the validation experiments in Figure 3, tumors were allowed to develop for 15 weeks after 847 viral delivery of a lentiviral pool that contained 26 barcoded Lenti-sgRNA/Cre vectors (Lenti$848 \mathrm{sgValidation} / \mathrm{Cre})$. Tumors were initiated in $\operatorname{Kras}^{\text {LSL-G12D; }}{ }^{\text {R26 }}$ LSL-tdTomato $^{\text {LT }}$ or $K T ; \mathrm{H} 11^{\text {LSL-Cas9; }}$ 849 mice with $3 \times 10^{5}$ ifu/mouse.

850 For the individual sgRNA tumor initiation experiments in Figure 3, tumors were allowed to 851 develop for 12 weeks after viral delivery of individual sgRNA expressing lentiviral vector that

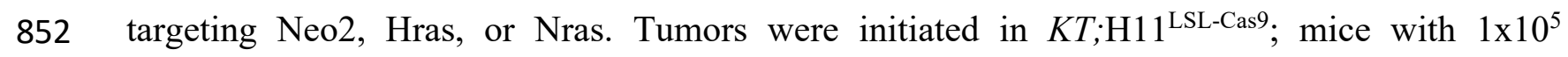
853 ifu/mouse.

854 For the paired screen experiments in Figure 6, tumors were allowed to develop for 15 weeks after 855 viral delivery of a lentiviral pool that contained 15 barcoded Lenti-sgRNA/Cre vectors (Lenti856 sgMultiGEMM/Cre). Tumors were initiated in $K T ; H 11^{L S L-C a s 9 /+}$ or $B r a f^{V 600 E} ; R 26^{L S L-}$ $857{ }^{\text {tdTomato }} ; H 11^{\text {LSL-Cas9/+ }}$ mice with $3 \times 10^{5}$ ifu/mouse. Note that $K T ; \mathrm{H} 11^{\text {LSL-Cas9/+ }}$ rather than KT;H1 $1^{\text {LSL- }}$ $858^{\text {Cas9/LSL-Cas9 }}$ mice were used in this experiment to match the Cas9 dosage of the BrafT;H1 $1^{\text {LSL-Cas9/+ }}$ 859 mice, whereas $K T ; \mathrm{H} 11^{\text {LSL-Cas9/LSL-Cas9 }}$ mice were used in all other experiments. To evaluate the 860 effects of Cas9 dosage on the tumor suppressive effects of the Lenti-sgMultiGEMM/Cre pool, we 861 also initiated tumors in a small cohort of $K T ; H 11^{L S L-C a s 9 / L S L-C a s 9}$ mice. Reductions in the magnitude 862 of the effects of various sgRNAs were observed in the KT;H11 $1^{\text {LSL-Cas } 9 /+}$ cohort relative to the $863 K T ; H 11^{L S L-C a s 9 / L S L-C a s 9}$ cohort, underscoring the importance of matching Cas9 dosage and 864 suggesting that Cas9 can be limiting in H11LSL-Cas9/+ mice. 
867 Genomic DNA was isolated from bulk tumor-bearing lung tissue from each mouse as previously

868 described. Briefly, benchmark control cell lines were generated from LSL-YFP MEFs transduced

869 by a barcoded Lenti-sgNT3/Cre vector (NT3: an inert sgRNA with a distinct sgID) and purified

870 by sorting YFP ${ }^{\text {pos }}$ cells. Three benchmark control cell lines (500,000 cells each) were added to

871 each mouse lung sample prior to lysis to enable the calculation of the absolute number of neoplastic

872 cells in each tumor from the number of sgID-BC reads. Following homogenization and overnight

873 protease K digestion, genomic DNA was extracted from the lung lysates using standard phenol-

874 chloroform and ethanol precipitation methods. Subsequently, Q5 High-Fidelity 2x Master Mix

875 (New England Biolabs, M0494X) was used to amplify the sgID-BC region from $32 \mu \mathrm{g}$ of genomic

876 DNA in a total reaction volume of $800 \mu \mathrm{l}$ per sample. The unique dual-indexed primers used were

877 Forward: AAT GAT ACG GCG ACC ACC GAG ATC TAC AC-8 nucleotides for i5 index-ACA

878 CTC TTT CCC TAC ACG ACG CTC TTC CGA TCT-6 to 9 random nucleotides for increased

879 diversity-GCG CAC GTC TGC CGC GCT G and Reverse: CAA GCA GAA GAC GGC ATA

880 CGA GAT-6 nucleotides for i7 index- GTG ACT GGA GTT CAG ACG TGT GCT CTT CCG

881 ATC T-9 to 6 random nucleotides for increased diversity-CAG GTT CTT GCG AAC CTC AT.

882 The PCR products were purified with Agencourt AMPure XP beads (Beckman Coulter, A63881)

883 using a double size selection protocol. The concentration and quality of the purified libraries were

884 determined using the Agilent High Sensitivity DNA kit (Agilent Technologies, 5067-4626) on the 885 Agilent 2100 Bioanalyzer (Agilent Technologies, G2939BA). The libraries were pooled based on

886 lung weight to ensure even reading depth, cleaned up again using AMPure XP beads, and 887 sequenced (read length $2 \times 150 \mathrm{bp}$ ) on the Illumina HiSeq 2500 or NextSeq 550 platform (Admera 888 Health Biopharma Services). 
891 Parental cells were seeded at 50\% confluency in a 6-well plate the day before transduction (day 892 0). The cell culture medium was replaced with $2 \mathrm{~mL}$ fresh medium containing $8 \mu \mathrm{g} / \mathrm{mL}$ 893 hexadimethrine bromide (Sigma Aldrich, H9268-5G), $20 \mu \mathrm{L}$ ViralPlus Transduction Enhancer 894 (Applied Biological Materials Inc., G698) and $40 \mu \mathrm{L}$ concentrated lentivirus and cultured 895 overnight (Day 1). The medium was then replaced with complete medium and cultured for another 89624 hours (Day 2). Cells were transferred into a $100 \mathrm{~mm}$ cell culture dish with appropriate amounts 897 of antibiotic (Blasticidin doses: U2OS: $10 \mu \mathrm{g} / \mathrm{mL}$; HOP62: $50 \mu \mathrm{g} / \mathrm{mL}$; H727: $10 \mu \mathrm{g} / \mathrm{mL}$; H23: 15 $898 \mu \mathrm{g} / \mathrm{mL}$; Puromycin doses: HC494: $5 \mu \mathrm{g} / \mathrm{mL}$; U2OS: $1 \mu \mathrm{g} / \mathrm{mL}$; HOP62: $5 \mu \mathrm{g} / \mathrm{mL}$; H727: $5 \mu \mathrm{g} / \mathrm{mL}$; $899 \mathrm{H} 23: 5 \mu \mathrm{g} / \mathrm{mL}$ ) and selected for 48 hours (Day 3).

900

901 Western Blot

902 Cells were lysed in RIPA buffer (50 mM Tris- $\mathrm{HCl}(\mathrm{pH} 7.4), 150 \mathrm{mM} \mathrm{NaCl}, 1 \%$ Nonidet P-40, and $9030.1 \%$ SDS) and incubated at $4{ }^{\circ} \mathrm{C}$ with continuous rotation for 30 minutes, followed by 904 centrifugation at $12,000 \times \operatorname{rcf}$ for 10 minutes. The supernatant was collected, and the protein 905 concentration was determined by BCA assay (Thermo Fisher Scientific, 23250). Protein extracts $906(10-50 \mu \mathrm{g})$ were dissolved in 10\% SDS-PAGE and transferred onto PVDF membranes. The 907 membranes were blocked with 5\% non-fat milk in TBS with $0.1 \%$ Tween 20 (TBST) at room 908 temperature for one hour, cut according to the molecular weight of target protein (with at least two 909 flacking protein marker), followed by incubation with primary antibodies diluted in TBST $910(1: 1000)$ at $4{ }^{\circ} \mathrm{C}$ overnight. After three 10-minutes washes with TBST, the membranes were 911 incubated with the appropriate secondary antibody conjugated to HRP diluted in TBST (1:10000) 912 at room temperature for 1 hour. After three 10-minutes washes with TBST, Protein expression 
913 was quantified with enhanced chemiluminescence reagents (Fisher Scientific, PI80196). For AKT

914 and ERK, phosphorylated proteins were detected first and the membrane were striped, blocked, 915 and incubated with $1^{\text {st }}$ and $2^{\text {nd }}$ antibodies for pan protein detections.

916 Antibodies used in this study: HSP90 (BD Biosciences, 610418), pAKT (Cell Signaling, 4060S), 917 pERK (Cell Signaling, 4370L), ERK (Cell Signaling, 9102S), AKT (Cell Signaling, 4691S), 918 HRAS (Thermo Fisher Scientific, 18295-1-AP), NRAS (Santa Cruz Biotechnology, sc-31), HA919 tag (Santa Cruz Biotechnology, sc-7392).

920

921 Histology and immunohistochemistry (IHC)

922 Lung lobes were fixed in $4 \%$ formalin and paraffin embedded. Hematoxylin and eosin staining 923 was performed using standard methods. IHC was performed on 4- $\mu \mathrm{m}$ sections with IHC was 924 performed using Avidin/Biotin Blocking Kit (Vector Laboratories, SP-2001), Avidin-Biotin 925 Complex kit (Vector Laboratories, PK-4001), and DAB Peroxidase Substrate Kit (Vector 926 Laboratories, SK-4100) following standard protocols.

927 The following primary antibodies were used: Ki-67 (BD Pharmingen, 550609), BrdU (BD 928 Pharmingen, 555627), human mitochondria (Abcam, ab92824), pERK (Cell Signaling, 4370L).

929 Total tumor burden (tumor area/total area $\times 100 \%$ ), mitochondria ${ }^{\text {pos }}$ tumor burden (mitochondria ${ }^{\text {pos }}$ 930 area/total area $\times 100 \%$ ), BrdU ${ }^{\text {pos }}$ cell number, Ki6 $7^{\text {pos }}$ cell number, and $\mathrm{pERK}{ }^{\text {pos }}$ cell number were 931 calculated using ImageJ.

932

\section{Cell proliferation assay (CCK8)}

934 For cell proliferation assays, cells were seeded in 96-well plates at a density of 5000 cells per well 935 and allowed to adhere overnight in regular growth media (Day 0). Cells were then cultured in 
936 media as indicated on each figure panel for 7 days. Relative cell number were measured every

937 other day using Cell Counting Kit-8 (Bimake, B34304) according to the manufacturer's

938 instructions.

939

940 Colony formation assay

941 For clonogenic assays, cells were seeded in 6-well plates at a density of 500 cells per well

942 and allowed to adhere overnight in regular growth media. Cells were then cultured in media as

943 indicated on each figure panel for 14 days. Growth media with or without drugs was replaced every

9442 days. At the end point, cells were stained with $0.5 \%$ crystal violet in $20 \%$ methanol. Colony

945 numbers were calculated using ImageJ

946

947 Allograft studies in immunocompromised mice

948 For intravenous transplants into immunocompromised NSG mice, $5 \times 10^{5} \mathrm{H} 23$ cells were injected 949 into one of lateral tail veins. Mice were sacrificed 28 days post-injection and lung lobe were fixed 950 in $4 \%$ formalin and paraffin embedded. For subcutaneous transplants into immunocompromised 951 NSG mice, $2 \times 10^{6}$ of each H23 cells (sgSAFE, sgHRAS, and sgNRAS) were re-suspended in 952 200uL Matrigel ${ }^{\circledR}$ Basement Membrane Matrix (Corning, 354234) and injected into three parallel 953 sites per mouse. Mice were sacrificed 28 days post-injection. Tumors were dissected and the 954 weight, height, width, and length, of each tumor was measured. Tumor volume was roughly 955 calculated via the formula: $\mathrm{V}=(4 / 3) \times \pi \times(\mathrm{L} / 2) \times(\mathrm{L} / 2) \times(\mathrm{D} / 2)$.

956 Institute of Medicine Animal Care and Use Committee approved all animal studies and procedures. 957

958 ReBiL2.0 assay 
959 ReBiL2.0 assay was performed as previously descried ${ }^{16}$. ReBiL cells (U2OS-134-764np or U2OS-

960 134-794p with overexpression of KRAS4b, HRAS or NRAS) were seeded in i) 96-well plates at

961 density of $2 \times 10^{4}$, and ii) 6-well plates at density of $1 \times 10^{6}$ and allowed to adhere overnight in regular

962 growth media (DMEM/F12, 10\% FBS, and $10 \mu \mathrm{g} / \mathrm{mL}$ ciprofloxacin). The next day, cells were

963 then cultured in serum limited media (DMEM/F12, 1\% FBS, and $10 \mu \mathrm{g} / \mathrm{mL}$ ciprofloxacin)

964 containing $100 \mathrm{ng} / \mathrm{mL}$ doxycycline for 24 hours. Upon termination of the ReBiL assay, i) to

965 measure raw luciferase activity, $300 \mu \mathrm{M}$ D-luciferin was added to 96-well plate culture and

966 incubate in $37^{\circ} \mathrm{C}$ for 30 mins and raw luminescent data collected by a Tecan microplate reader; ii)

967 to measure viable cell numbers, CCK-8 assay were performed in the same 96 -well plate culture

968 and raw cell number data collected by a Tecan microplate reader; iii) to quantify the $1 / 2$ luc fusion

969 proteins, ReBiL cells from 6-well plate culture were harvested with RIPA lysis buffer for protein

970 extraction and western blot was performed for HA-tag and HSP90 expression. Then the ReBiL2.0

971 score was calculated via the formula:

$972 \operatorname{ReBiL2.0~score~}=([$ Raw Luminescence $] /[$ Cell number $]) /([1 / 2$ luc Least $] /[$ HSP90 $])$

973

974 Analysis of human lung adenocarcinoma cancer genome sequencing data (for HRAS rare

975 mutations)

976 To assess evidence that HRAS functions as a Kras-specific tumor suppressor in human cancer, we

977 queried publicly available cancer genomic datasets. GENIE Release 9.1-public was accessed

978 through the Synapse platform and data on somatic mutations (data_mutations_extended.txt), 979 sample-and patient-level clinical data (data_clinical_sample.txt and data_clinical_patient.txt), and

980 genotyping panel information (genomic_information.txt) were downloaded. While it is unclear

981 how our findings may extrapolate to cancer types beyond lung adenocarcinoma, Hras mutations 
982 are exceedingly rare (occurring at a frequency of just $\sim 0.008$ in GENIE samples) so we performed

983 a pan-cancer analysis. Each sample was assigned to its patient of origin and annotated for the

984 presence of both oncogenic Kras mutations (defined as missense mutations in Kras exons 12, 13

985 or 61) and for the presence of potentially functional Hras mutations (variants that were silent,

986 intergenic, intronic, or fell in the $3^{\prime}$ or 5' UTRs were excluded from this analysis). When multiple

987 samples were derived from the same patient, the patient in question was annotated as having a

988 mutation if it occurred in at least one of their associated samples. From this information we

989 produced a list of the frequency of all Hras variants in patients with and without oncogenic Kras

990 in both datasets. The genotyping panel information was used to identify GENIE patients that were

991 not genotyped at Hras and exclude these from the frequency calculation.

992

993 Process paired-end reads to identify the sgID and barcode

994 Sequencing of Tuba-seq libraries produces reads that are expected to contain an 8-nucleotide sgID 995 followed by a 30-nucleotide barcode (BC) of the form

996 GCNNNNNTANNNNNGCNNNNNTANNNNNGC, where each of the 20 Ns represent random 997 nucleotides. Each sgID has a one-to-one correspondence with an sgRNA in the viral pool; thus,

998 the sgID sequence identifies the gene targeted in a given tumor. Note that all sgID sequences in 999 the viral pool differ from each other by at least three nucleotides such that incorrect sgID 1000 assignment (and thus, inference of tumor genotype) due to PCR or sequencing error is extremely 1001 unlikely. The random 20-nucleotide portion of the $\mathrm{BC}$ is expected to be unique to each lentiviral 1002 integration event, and thus tags all cells in a single clonal expansion. Note that the length of the 1003 barcode ensures a high theoretical potential diversity $\left(\sim 4^{20}>10^{12}\right.$ barcodes per vector), so while 1004 the actual diversity of each Lenti-sgRNA/Cre vector is dictated by the number of colonies 
1005 generated during the plasmid barcoding step, it is very unlikely that we will observe the same BC 1006 in multiple clonal expansions.

1007 FASTQ files were parsed using regular expressions to identify the sgID and BC for each read. To 1008 minimize the effects of sequencing error on $\mathrm{BC}$ identification, we required the forward and reverse 1009 reads to agree completely within the 30 -nucleotide sequence to be further processed. We also 1010 screened for barcodes that were likely to have arisen due to errors in sequencing the barcodes of 1011 genuine tumors. Given the low rate of sequencing error, we expect these spurious "tumors" to have 1012 read counts that are far lower than the read counts of the genuine tumors from which they arise. 1013 While it is impossible to eliminate these spurious tumors, we sought to minimize their effect by 1014 identifying small "tumors" with barcodes that are highly similar to the barcodes of larger tumors. 1015 Specifically, if a pair of "tumors" had barcodes that were within a Hamming distance of two, and 1016 if one of the tumors had less than 5\% as many reads as the other, then the reads associated with 1017 the smaller tumor were attributed to the larger tumor.

1018 After these filtering steps, the read counts associated with each barcode were converted to absolute 1019 neoplastic cell numbers by normalizing to the number of reads in the "spike-in" cell lines added 1020 to each sample prior to lung lysis and DNA extraction. The median sequencing depth across 1021 experiments was $\sim 1$ read per 6.4 cells.

1022 For statistical comparisons of tumor genotypes, we applied a minimum tumor size cutoff of 100 1023 cells. In selecting a cutoff, we sought to include tumors that are large enough to be consistently 1024 detected despite differences in sequencing depth among mice, while using as many tumors as 1025 possible to maximize the statistical power. Importantly, we analyzed each Tuba-seq dataset with 1026 multiple minimum tumor size cut-offs $(50,100,200,500$ cells $)$ and found that our findings were 1027 robust. 
1029 Summary statistics for overall growth rate

1030 To assess the extent to which a given gene $(X)$ affects tumor growth, we compared the distribution 1031 of tumor sizes produced by vectors targeting that gene ( $\operatorname{sg} X$ tumors) to the distribution produced 1032 by our negative control vectors (sgInert tumors). We relied on two statistics to characterize these 1033 distributions: the size of tumors at defined percentiles of the distribution (specifically the $50^{\text {th }}, 60^{\text {th }}$, $103470^{\text {th }}, 80^{\text {th }}, 90^{\text {th }}$, and $95^{\text {th }}$ percentile tumor sizes), and the log-normal mean size (LN mean). The 1035 percentile sizes are nonparametric summary statistics of the tumor size distribution. In considering 1036 percentiles corresponding to the right tail of the distribution, we focus on the growth of larger 1037 tumors, thereby avoiding issues stemming from potential variation in cutting efficiency among 1038 guides. The LN mean is the maximum-likelihood estimate of mean tumor size assuming a log1039 normal distribution. Previous work found that this statistic represents the best parametric summary 1040 of tumor growth based on the maximum likelihood quality of fit of various common parametric 1041 distributions.

1043 To quantify the extent to which each gene suppressed or promoted tumor growth, we normalized 1044 statistics calculated on tumors of each genotype to the corresponding inert statistic. The resulting 1045 ratios reflect the growth advantage (or disadvantage) associated with each tumor genotype relative 1046 to the growth of sgInert tumors.

1048 For example, the relative $\mathrm{i}^{\text {th }}$ percentile size for tumors of genotype $\mathrm{X}$ was calculated as:

1049

1050

Relative size at $i^{\text {th }}$ percentile sgX $=\frac{i^{\text {th }} \text { percentile of } \operatorname{sgX} \text { distribution }}{i^{\text {th }} \text { percentile of s.gInert distribution }}$ 
1052 Likewise, the relative LN mean size for tumors of genotype $\mathrm{X}$ was calculated as:

1053

1054

Relative $L_{N m e a n_{s g X}}=\frac{\text { LNmean of } s g X \text { distribution }}{\text { LNmean of s.gInert distributior }}$

1055

1056 Summary statistics for relative tumor number and relative tumor burden

1057 In addition to the tumor size metrics described above, we characterized the effects of gene 1058 inactivation on tumorigenesis in terms of the number of tumors and total neoplastic cell number 1059 ("tumor burden") associated with each genotype. Unlike the aforementioned metrics of tumor size, 1060 tumor number and burden are linearly affected by lentiviral titer and are thus sensitive to 1061 underlying differences in the representation of each Lenti-sgRNA/Cre vector in the viral pool. 1062 Critically, each Tuba-seq experiment included a cohort of $K T$ control mice. $K T$ mice lack 1063 expression of Cas9, thus all Lenti-sgRNA/Cre vectors are functionally equivalent in these mice, 1064 and the observed tumor number and burden associated with each sgRNA reflects the make-up of 1065 the viral pool.

1066 To assess the extent to which a given gene $(X)$ affects tumor number, we therefore first normalized 1067 the number of $\operatorname{sgX}$ tumors in $K T ; H 11^{L S L-C a s 9}$ mice (also KT;p5 $3^{\text {flox/flox }} ; H 11^{L S L-C a s 9}$ and Braf ${ }^{L S L-}$ $1068{ }^{\mathrm{V} 600 E /+} T ; H 11^{L S L-C a s 9}$ mice in the initial Kras-interacting protein screen and the paired screen, 1069 respectively) to the number of $\operatorname{sgX}$ tumors in the $K T$ mice:

1070

1071

$$
\text { Tumor number } \text { T }_{\text {IX }}=\frac{\text { Number of } s g X \text { tumors in } K T ; H 11^{\text {LSL-Cas } 9} \text { mice }}{\text { Number of saX tumors in KT mice }}
$$


1073 As with the tumor size metrics, we then calculated a relative tumor number by normalizing this

1074 statistic to the corresponding statistic calculated using sgInert tumors:

1075

1076

Relative tumor number $_{\text {sgX }}=\frac{\text { Tumor number }_{\text {sgX }}}{\text { Tumor number }}$

1078 Genes that influence relative tumor number modify the probability of tumor initiation and/or the 1079 very early stages of oncogene-driven epithelial expansion, which prior work suggests are 1080 imperfectly correlated with tumor growth at later stages. Relative tumor number thus captures an 1081 additional and potentially important aspect of tumor suppressor gene function.

1082

1083 Analogous to the calculation of relative tumor number, we characterized the effect of each gene 1084 on tumor burden by first normalizing the sgX tumor burden in Cas9-expressing mice to the burden 1085 in KT mice:

1086

1087

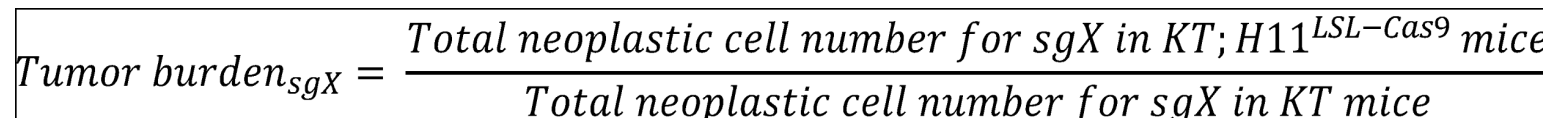

1088

1089 We then calculated a relative tumor burden by normalizing this number to the corresponding 1090 statistic calculated using sgInert tumors:

1091

1092

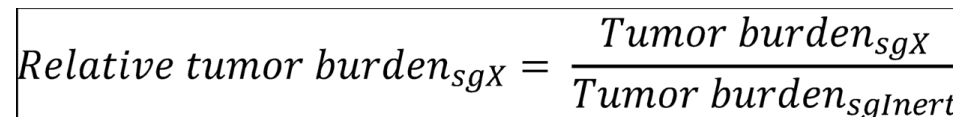


1094 Tumor burden is an integration over tumor size and number, and thus reflects the total neoplastic 1095 load in each mouse. Tumor burden is thus more strongly related to morbidity than are our metrics 1096 of tumor size and is closely related to traditional measurements of tumor progression such as 1097 duration of survival and tumor area. While intuitively appealing, tumor burden is notably nosier 1098 than our metrics of tumor size as it is strongly determined by the size of the largest tumors.

1100 Calculation of confidence intervals and P-values for tumor growth and number metrics

1101 Confidence intervals and $P$-values were calculated using bootstrap resampling to estimate the 1102 sampling distribution of each statistic. To account for both mouse-to-mouse variability and 1103 variability in tumor size and number within mice, we adopted a two-step, nested bootstrap 1104 approach where we first resampled mice, and then resampled tumors within each mouse in the 1105 pseudo-dataset. 10,000 bootstrap samples were drawn for all reported P-values. 95\% confidence 1106 intervals were calculated using the $2.5^{\text {th }}$ and $97.5^{\text {th }}$ percentile of the bootstrapped statistics. Because 1107 we calculate metrics of tumor growth that are normalized to the same metrics in sgInert tumors, 1108 under the null model where genotype does not affect tumor growth, the test statistic is equal to 1. 1109 Two-sided p-values were thus calculated as followed: $p=2 * \min \{\operatorname{Pr}(T>1), \operatorname{Pr}(T<1)\}$

1111 Where $\mathrm{T}$ is the test statistic and $\operatorname{Pr}(\mathrm{T}>1)$ and $\operatorname{Pr}(\mathrm{T}<1)$ were calculated empirically as the proportion

1112 of bootstrapped statistics that were more extreme than the baseline of 1 . To account for multiple 1113 hypothesis testing, p-values were FDR-adjusted using the Benjamini-Hochberg procedure as 1114 implemented in the Python package stats models. 
1117 AP-MS data was analyzed as described (Ding et al 2016). Briefly, protein spectral 1118 matches (PSMs; Kelly et al 2020) were normalized by protein length and total spectral matches

1119 per experiment. These normalized spectral abundance factors (NSAFs) were then normalized to 1120 NSAFs of matched prey proteins from a large cohort of unrelated AP/MS experiments to produce

1121 a Z-score. Z-scores are proportional to the areas of circles in bubble plots. In cluster diagrams,

1122 NSAFs are binarized by statistical significance (FDR $>0.5$ ), similarities between interactome

1123 profiles are determined by cosine distance, and dendrogram topology is determined by UPGMA.

1125 Modeling RAS-RAS dimer

1126 Potential templates for modeling the heterodimers were obtained from the ProtCID database.

1127 ProtCID is built from clustering interfaces of homologous proteins obtained from domain-domain

1128 contacts within protein crystals in the Protein Data Bank. Hierarchical clustering of interfaces is

1129 performed with a Jaccard-index similarity metric based on the contacts shared between different

1130 interfaces. Models for the structure of the HRAS/KRAS heterodimer were built by superposing a

1131 structure of KRAS-G12D (PDB: 5USJ) onto a monomer of the HRAS homodimer in PDB entry $11323 \mathrm{~K} 8 \mathrm{Y}$.

1133

1134

1135

1136

1137

1138

1139 
1141 We thank the Stanford Veterinary Animal Care Staff for expert animal care, Human 1142 Pathology/Histology Service Center, Stanford Protein and Nucleic Acid Facility for experimental 1143 support; A. Orantes for administrative support; Members of the Winslow laboratory and Ian Prior 1144 for helpful comments. R.T. was supported by a Stanford University School of Medicine Dean's 1145 Postdoctoral Fellowship, a Tobacco-Related Disease Research Program (TRDRP) Postdoctoral 1146 fellowship (27FT-0044), and a Stanford Cancer Institute fellowship. C.W.M. was supported by 1147 the NSF Graduate Research Fellowship Program and an Anne T. and Robert M. Bass Stanford 1148 Graduate Fellowship. J.D.H was supported by a Stanford University School of Medicine Dean's 1149 Postdoctoral Fellowship and a TRDRP Postdoctoral fellowship (T31FT1619). H.C. was supported 1150 by a TRDRP Postdoctoral Fellowship (28FT-0019). N.W.H. was supported by the NSF Graduate 1151 Research Fellowship Program. Work in the laboratory of G.M.W. was supported, in part, by 1152 Cancer Center Core Grant CA014195, the Breast Cancer Research Foundation, the Freeberg 1153 Foundation and the NIH/National Cancer Institute (Grant R35 CA197687). This work was 1154 supported by NIH R01-CA230025 (to M.M.W), NIH R01-CA231253 (to M.M.W and D.A.P), 1155 NIH R01-CA234349 (to M.M.W and D.A.P.), TRDRP 27IP-0052 (to M.M.W), and in part by the 1156 Stanford Cancer institute support grant (NIH P30-CA124435). 


\section{REFERENCES :}

1164 1. Karnoub, A.E. \& Weinberg, R.A. Ras oncogenes: split personalities. Nature reviews 1165 Molecular cell biology 9, 517-531 (2008).

$11662 . \quad$ Cox, A.D., Fesik, S.W., Kimmelman, A.C., Luo, J. \& Der, C.J. Drugging the undruggable 1167 RAS: mission possible? Nature reviews Drug discovery 13, 828-851 (2014).

1168 3. Zhou, B., Der, C.J. \& Cox, A.D. in Seminars in cell \& developmental biology, Vol. 58 60-69 1169 (Elsevier, 2016).

1170 4. Wennerberg, K., Rossman, K.L. \& Der, C.J. The Ras superfamily at a glance. Journal of cell 1171 science 118, 843-846 (2005).

1172 5. Hobbs, G.A., Der, C.J. \& Rossman, K.L. RAS isoforms and mutations in cancer at a glance. 1173 Journal of cell science 129, 1287-1292 (2016).

1174 6. Stephen, A.G., Esposito, D., Bagni, R.K. \& McCormick, F. Dragging ras back in the ring. 1175 Cancer cell 25, 272-281 (2014).

1176 7. Brose, M.S. et al. BRAF and RAS mutations in human lung cancer and melanoma. Cancer 1177 research 62, 6997-7000 (2002).

1178 8. Prior, I.A., Lewis, P.D. \& Mattos, C. A comprehensive survey of Ras mutations in cancer. 1179 Cancer research 72, 2457-2467 (2012).

1180 9. Papke, B. \& Der, C.J. Drugging RAS: Know the enemy. Science 355, 1158-1163 (2017).

1181 10. Kelly, M.R. et al. Combined proteomic and genetic interaction mapping reveals new RAS 1182 effector pathways and susceptibilities. Cancer discovery 10, 1950-1967 (2020).

1183 11. Broyde, J. et al. Oncoprotein-specific molecular interaction maps (SigMaps) for cancer 1184 network analyses. Nature biotechnology 39, 215-224 (2021).

1185 12. Zhou, Y. \& Hancock, J.F. Deciphering lipid codes: K-Ras as a paradigm. Traffic 19, 1571186165 (2018).

1187 13. Wittinghofer, A. \& Pal, E.F. The structure of Ras protein: a model for a universal 1188 molecular switch. Trends in biochemical sciences 16, 382-387 (1991).

1189 14. Omerovic, J., Hammond, D.E., Clague, M.J. \& Prior, I.A. Ras isoform abundance and 1190 signalling in human cancer cell lines. Oncogene 27, 2754-2762 (2008).

1191 15. Han, K. et al. CRISPR screens in cancer spheroids identify 3D growth-specific vulnerabilities. Nature 580, 136-141 (2020).

1193 16. Li, Y.-C. et al. Analysis of RAS protein interactions in living cells reveals a mechanism for 1194 1195 pan-RAS depletion by membrane-targeted RAS binders. Proceedings of the National Academy of Sciences 117, 12121-12130 (2020).

17. Hingorani, S.R. et al. Preinvasive and invasive ductal pancreatic cancer and its early detection in the mouse. Cancer cell 4, 437-450 (2003).

18. Cai, H. et al. A functional taxonomy of tumor suppression in oncogenic KRAS-driven lung cancer. Cancer Discovery (2021).

$120019 . \quad$ Rogers, Z.N. et al. Mapping the in vivo fitness landscape of lung adenocarcinoma tumor 1201 suppression in mice. Nature genetics 50, 483-486 (2018).

1202 20. Rogers, Z.N. et al. A quantitative and multiplexed approach to uncover the fitness 1203 landscape of tumor suppression in vivo. Nature methods 14, 737-742 (2017). 
1204 21. Chuang, C.-H. et al. Molecular definition of a metastatic lung cancer state reveals a 1205 targetable CD109-Janus kinase-Stat axis. Nature medicine 23, 291-300 (2017).

1206 22. Ruiz, S., Santos, E. \& Bustelo, X.R. RasGRF2, a guanosine nucleotide exchange factor for 1207 Ras GTPases, participates in T-cell signaling responses. Molecular and cellular biology 27, 812712088142 (2007).

1209 23. Brandt, A.C., Koehn, O.J. \& Williams, C.L. SmgGDS: An Emerging Master Regulator of 1210 Prenylation and Trafficking by Small GTPases in the Ras and Rho Families. Frontiers in Molecular 1211 Biosciences 8, 542 (2021).

1212 24. Rowell, C.A., Kowalczyk, J.J., Lewis, M.D. \& Garcia, A.M. Direct demonstration of 1213 geranylgeranylation and farnesylation of Ki-Ras in vivo. Journal of Biological Chemistry 272, 1214 14093-14097 (1997).

1215 25. Zhang, F.L. et al. Characterization of Ha-ras, N-ras, Ki-Ras4A, and Ki-Ras4B as in vitro 1216 substrates for farnesyl protein transferase and geranylgeranyl protein transferase type I. 1217 Journal of Biological Chemistry 272, 10232-10239 (1997).

1218 26. Takaya, A. et al. R-Ras regulates exocytosis by Rgl2/Rlf-mediated activation of RalA on 1219 endosomes. Molecular biology of the cell 18, 1850-1860 (2007).

1220 27. Marais, R., Light, Y., Paterson, H. \& Marshall, C. Ras recruits Raf-1 to the plasma 1221 1222 membrane for activation by tyrosine phosphorylation. The EMBO journal 14, 3136-3145 (1995). 28. Campbell, J.D. et al. Distinct patterns of somatic genome alterations in lung adenocarcinomas and squamous cell carcinomas. Nature genetics 48, 607-616 (2016). 29. Sánchez-Rivera, F.J. et al. Rapid modelling of cooperating genetic events in cancer through somatic genome editing. Nature 516, 428-431 (2014).

30. Kohl, N.E. et al. Selective inhibition of ras-dependent transformation by a farnesyltransferase inhibitor. Science 260, 1934-1937 (1993).

31. Rowinsky, E.K., Windle, J.J. \& Von Hoff, D.D. Ras protein farnesyltransferase: a strategic target for anticancer therapeutic development. Journal of Clinical Oncology 17, 3631-3652 (1999).

1231

1232

32. Collisson, E. et al. Comprehensive molecular profiling of lung adenocarcinoma: The cancer genome atlas research network. Nature 511, 543-550 (2014).

1233

1234 33. Feldser, D.M. et al. Stage-specific sensitivity to p53 restoration during lung cancer

1235 progression. Nature 468, 572-575 (2010).

34. Murray, C.W. et al. An LKB1-SIK axis suppresses lung tumor growth and controls differentiation. Cancer discovery 9, 1590-1605 (2019).

1238

1239 35. Tsherniak, A. et al. Defining a cancer dependency map. Cell 170, 564-576. e516 (2017). 36. Staffas, A., Karlsson, C., Persson, M., Palmqvist, L. \& Bergo, M. Wild-type KRAS inhibits oncogenic KRAS-induced T-ALL in mice. Leukemia 29, 1032-1040 (2015). 37. Ambrogio, C. et al. KRAS dimerization impacts MEK inhibitor sensitivity and oncogenic

1242 38. Kong, G. et al. Loss of wild-type Kras promotes activation of all Ras isoforms in 1243 oncogenic Kras-induced leukemogenesis. Leukemia 30, 1542-1551 (2016).

1244 39. Burgess, M.R. et al. KRAS allelic imbalance enhances fitness and modulates MAP kinase 1245 dependence in cancer. Cell 168, 817-829. e815 (2017). 
40. Young, A., Lou, D. \& McCormick, F. Oncogenic and wild-type Ras play divergent roles in the regulation of mitogen-activated protein kinase signaling. Cancer discovery 3, 112-123 (2013).

41. Grabocka, E. et al. Wild-type H-and N-Ras promote mutant K-Ras-driven tumorigenesis by modulating the DNA damage response. Cancer cell 25, 243-256 (2014). nanoclusters. Molecular and cellular biology 34, 862-876 (2014). Biochimica et Biophysica Acta (BBA)-Molecular Cell Research 1853, 841-849 (2015). nanoclusters. Molecular membrane biology 26, 80-92 (2009). Raf-1 activation. Journal of Biological Chemistry 275, 3737-3740 (2000). (2015). interface. Proceedings of the National Academy of Sciences 111, 2996-3001 (2014). 1585-1593 (2012). pathway. Proceedings of the National Academy of Sciences 112, 7996-8001 (2015). the impact on oncogenic ras signaling. Molecular cell 76, 872-884. e875 (2019). of BRAFV600E-induced lung tumors. Genes \& development 21, 379-384 (2007). prognostic marker and contributes to sorafenib resistance in hepatocellular carcinoma. Neoplasia 21, 257-268 (2019).

53. Weyandt, J.D. et al. Wild-type Hras suppresses the earliest stages of tumorigenesis in a genetically engineered mouse model of pancreatic cancer. PloS one 10, e0140253 (2015). 54. To, M.D., Rosario, R., Westcott, P.M., Banta, K.L. \& Balmain, A. Interactions between wild-type and mutant Ras genes in lung and skin carcinogenesis. Oncogene 32, 4028-4033 (2013).

55. Weyandt, J.D., Carney, J.M., Pavlisko, E.N., Xu, M. \& Counter, C.M. Isoform-Specific effects of wild-type ras genes on carcinogen-Induced lung tumorigenesis in mice. Plos one 11, e0167205 (2016). oncogenic Ras is essential for tumorigenesis. Nature communications 3, 1-8 (2012).

1286 58. Xu, Q. \& Dunbrack, R.L. ProtCID: a data resource for structural information on protein 1287 interactions. Nature communications 11, 1-16 (2020). 
1288 59. Alford, R.F. et al. The Rosetta all-atom energy function for macromolecular modeling 1289 and design. Journal of chemical theory and computation 13, 3031-3048 (2017).

1290 60. Shapovalov, M.V. \& Dunbrack Jr, R.L. A smoothed backbone-dependent rotamer library 1291 for proteins derived from adaptive kernel density estimates and regressions. Structure 19, 8441292858 (2011).

1293 
Tang, Shuldiner et al.

a

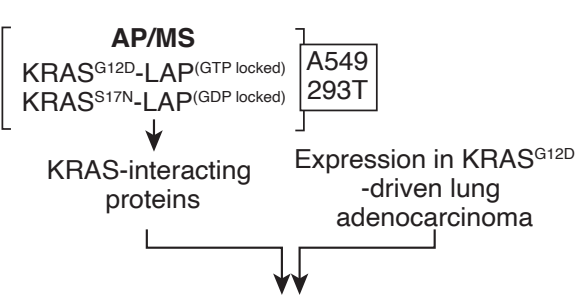

Candidate regulators of KRAS ${ }^{\mathrm{G} 12 \mathrm{D}}$-driven tumor growth b

\begin{tabular}{cccc} 
Protein & $\begin{array}{c}\text { KRAS } \\
\text { GTP }\end{array}$ & $\begin{array}{c}\text { KRAS } \\
\text { GDP }\end{array}$ & $\begin{array}{c}\text { Exp } \\
\text { (TPM) }\end{array}$ \\
\hline ALDH1A1 & $4.0 \times 10^{-7}$ & $1.1 \times 10^{-7}$ & 176.9 \\
CAND1 & $4.7 \times 10^{-3}$ & $1.1 \times 10^{-2}$ & 13.6 \\
FNTA & $1.9 \times 10^{-6}$ & $1.2 \times 10^{-6}$ & 34.7 \\
HRAS & $2.4 \times 10^{-12}$ & $3.0 \times 10^{-7}$ & 32.4 \\
NME2 & $2.3 \times 10^{-3}$ & $7.2 \times 10^{-3}$ & 26.1 \\
NRAS & $4.9 \times 10^{-6}$ & $2.9 \times 10^{-4}$ & 51.1 \\
RADIL & $6.4 \times 10^{-6}$ & ND & 6.2 \\
RAF1 & $5.6 \times 10^{-6}$ & ND & 21.5 \\
RAP1GDS1 & ND & $5.5 \times 10^{-6}$ & 10 \\
RAP1B & $1.6 \times 10^{-6}$ & ND & 162.4 \\
RASGRF2 & ND & $1.9 \times 10^{-11}$ & 0.9 \\
RGL2 & $1.2 \times 10^{-5}$ & ND & 8.6 \\
STOM & $6.0 \times 10^{-8}$ & ND & 15.8 \\
& & &
\end{tabular}

C

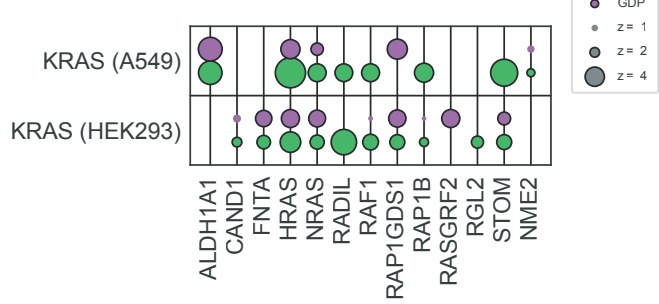

d

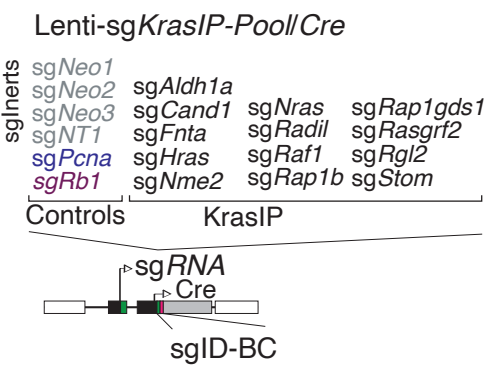

Lenti-sgKras/P-Pool/Cre

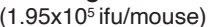

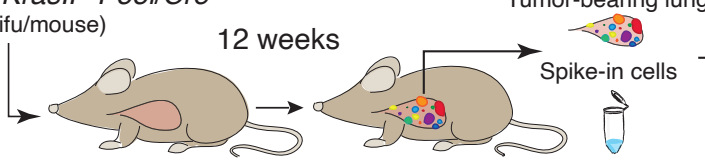

$\operatorname{Kras}^{L S L-G 12 D /+} ;$ Rosa26 $6^{L S L-T o m}(K T ; \mathrm{N}=5)$ $K T ; H_{11}{ }^{L S L-C a s 9}(\mathrm{~N}=12)$

$K T ; p 53^{\text {flloxflox }} ; H_{1} 1^{\text {LLL-Cas9 }}(\mathrm{N}=5)$
Tuba-seq

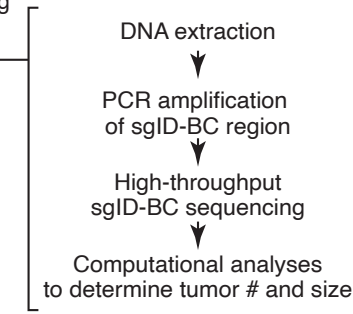

f

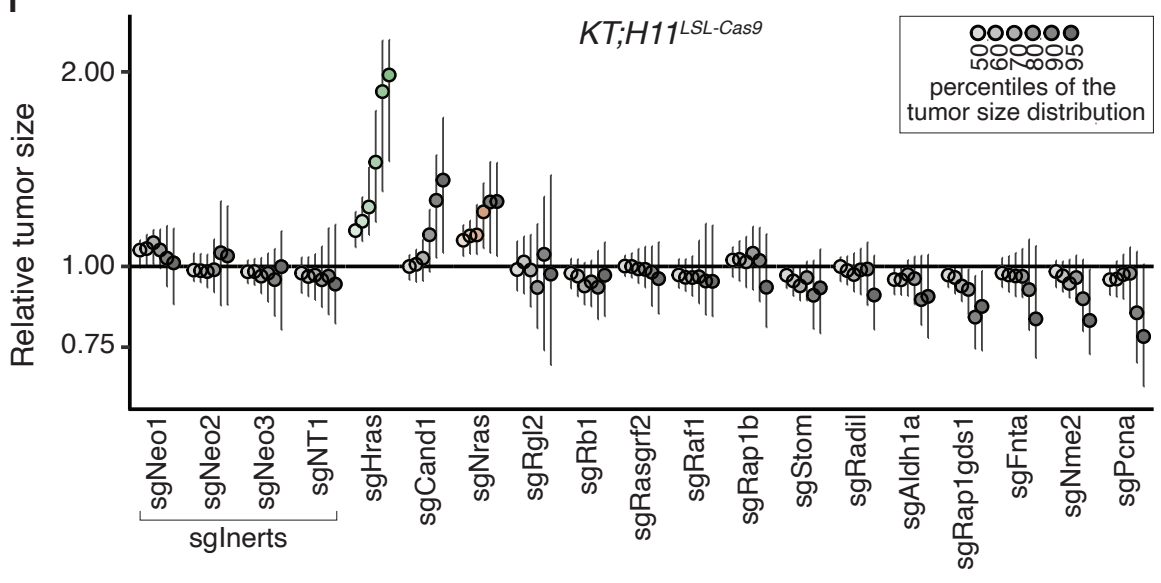

g

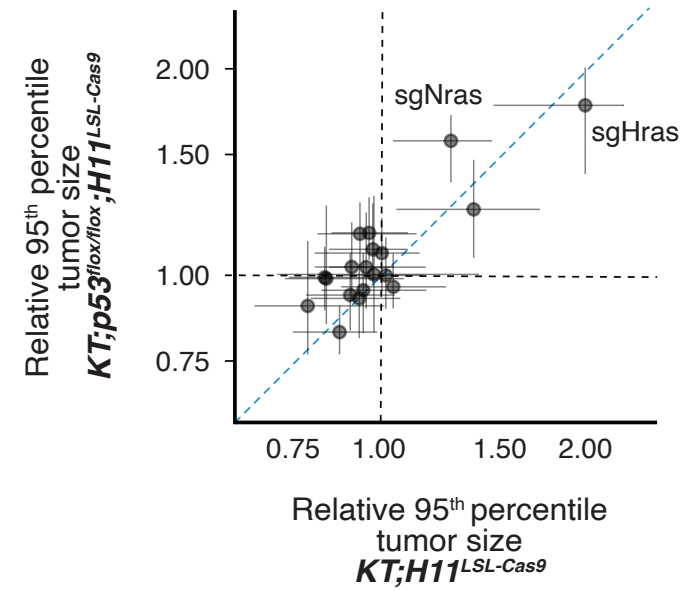

Figure 1. Multiplexed identification of KRAS-interacting proteins that impact KRAS ${ }^{\mathrm{G} 12 \mathrm{D}}$-driven lung cancer growth in vivo.

a. Candidate mediators of KRAS-driven lung tumor growth were identified on the basis of their interactions with GTP- and GDP-locked Kras in multiple AP/MS-based protein-protein interaction screens and their expression in a mouse model of Kras-driven lung adenocarcinoma.

b. Selected KRAS-interacting proteins interact with either GTP- or GDP-locked KRAS (shown as NSAF in A549 cells) and their homolog is expressed in KRAS ${ }^{\mathrm{G} 12 \mathrm{D}}$-driven lung cancer (shown as TPM).

c. Bubble plot of two AP/MS experiments with GTP- and GDP-locked mutant GTPases as baits (rows), showing the enrichment of selected candidate KRAS-interacting proteins (columns). Dark borders indicate FDR $<0.05$.

d. Schematic of tumor initiation with a pool of barcoded Lenti-sgRNA/Cre vectors (Lenti-sgKrasIP-Pool/Cre). The lentiviral pool includes four Inert sgRNAs that are either non-targeting (NT) or target a functionally inert locus (Neo1-3, targeting NeoR in the R26 LLL-tdTomato allele). Each barcoded lentiviral vector contains an sgRNA, Cre, and a two-component barcode composed of an sgRNA identifier (sgID) and a random barcode (BC). This design allows inactivation of multiple target genes in parallel followed by quantification of the resulting tumor size distributions through high-throughput sglD-BC sequencing.

e. Tumors were initiated in cohorts of $K T, K T ; H 11^{L S L-C a s 9}$ and $K T ;$; $53^{\text {floxflox } ; H 11^{L S L} \text {-Cas9 }}$ mice through intratracheal delivery of Lenti-sgKrasIP-Pool/Cre. Tuba-seq was performed on each tumor-bearing lung 12 weeks after initiation, followed by analyses of sgID-BC sequencing data to characterize the effects of inactivating each gene.

f. Tumor sizes at indicated percentiles for each sgRNA relative to the size of sglnert-containing tumors at the corresponding percentiles in $K T$; H11 ${ }^{L S L-C a s 9}$ mice. Genes are ordered by $95^{\text {th }}$ percentile tumor size, with sglnerts on the left. sglnerts are in gray, and the line at $y=1$ indicates no effect relative to sglnert. Error bars indicate $95 \%$ confidence intervals. Percentiles that are signficantly different from sglnert (two-sided FDR-adjusted $p<0.05$ ) are in color. Confidence intervals and $\mathrm{P}$-values were calculated by bootstrap resampling.

g. Comparison of $95^{\text {th }}$ percentile tumor size for each sgRNA relative to the size the $95^{\text {th }}$ percentile tumor size of sglnert-containing tumors in $K T$; $H 11^{1 S L-C a s 9}$ mice versus $K T$; $553^{\text {floxfflox}} ; H 11^{\text {LLLCas9 }}$ mice. Error bars indicate $95 \%$ confidence intervals calculated by bootstrap resampling. 
a

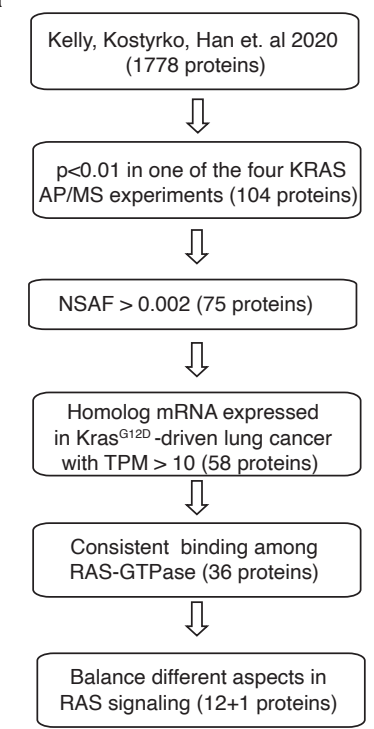

d

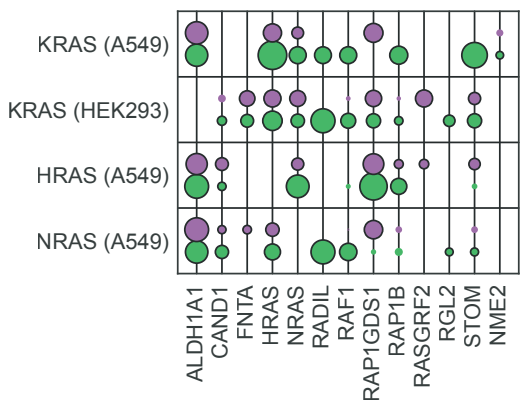

b

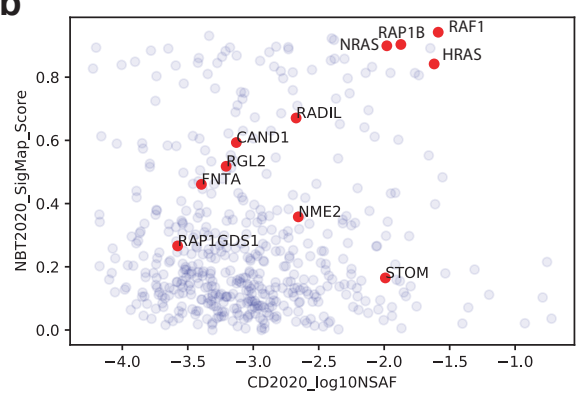

C

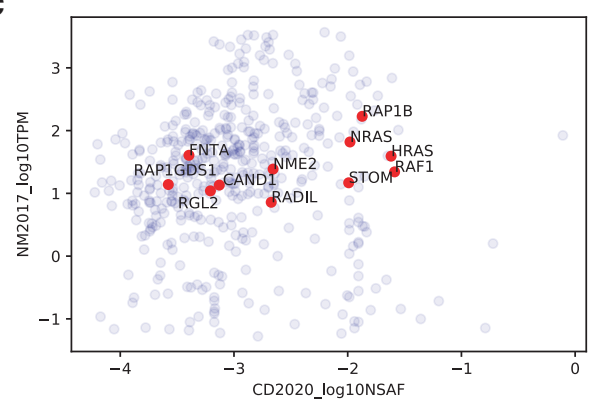

e

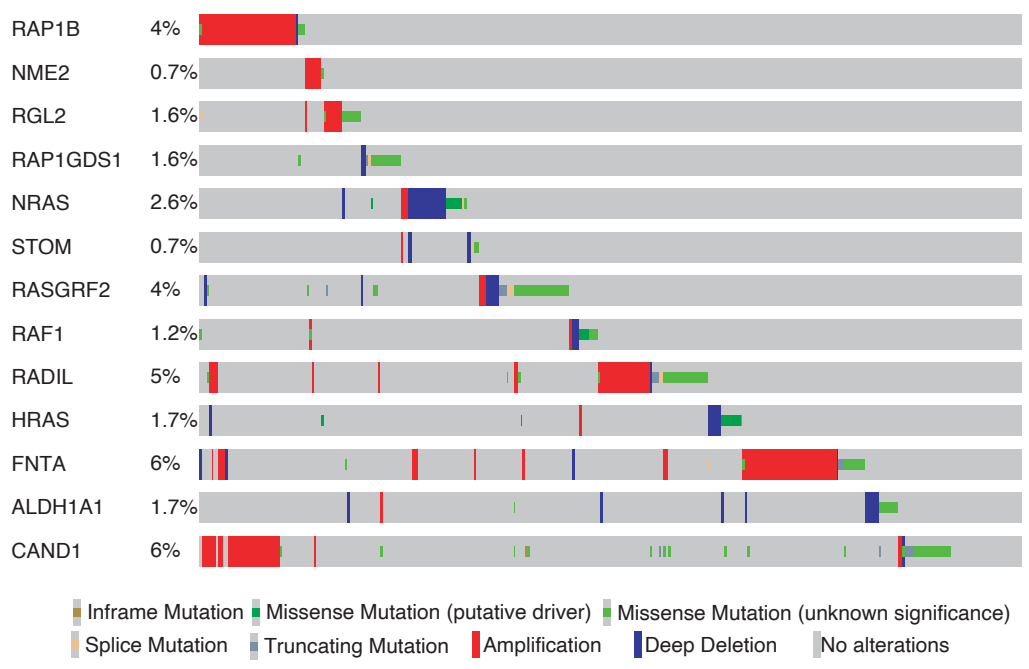

Supplemental Figure 1. Prioritize candidate KRAS-interacting proteins for this study.

a. Flow chart for prioritizing candidate KRAS-interacting proteins for this study. Candidate KRAS-interacting proteins were chosen based on multiple criteria including their interaction with KRAS, their homolog mRNA expression in Kras ${ }^{\mathrm{G} 12 \mathrm{D}}$-driven lung cancer in mouse model, and the consistency for them to bind different RAS-GTPase. RADIL is added at the last step due to its validated importance in KRAS-mutant human cell lines.

b. Candidate proteins interact with KRAS from two protein-protein interaction analyses (Kelly, Kostyrko, Han et al. 2020; Broyde,

Simpson, Murray et al. 2020). Shared KRAS-interaction proteins are shown as their $\log _{10}$ NSAF and SigMap Score.

c. Homolog mRNA expression (TPM) of candidate KRAS-interacting proteins in Kras ${ }^{\mathrm{G} 12 \mathrm{D}}$-driven lung cancer in mouse model (Chuang et al. 2017).

d. Bubble plot of eight AP/MS experiments with GTP- and GDP-locked mutant GTPases as baits (rows), showing the enrichment of selected candidate KRAS-interacting proteins (columns). Dark borders indicate FDR $<0.05$.

e. Mutation frequencies of these 13 candidate genes in lung adenocarcinoma (data from TCGA, Nat. Genet. 2016). 
a

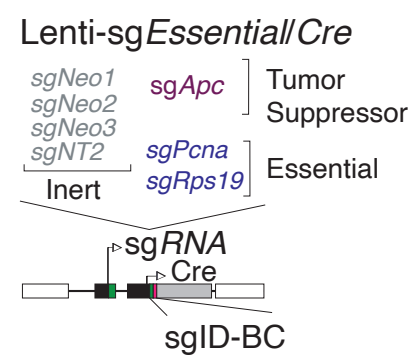

C

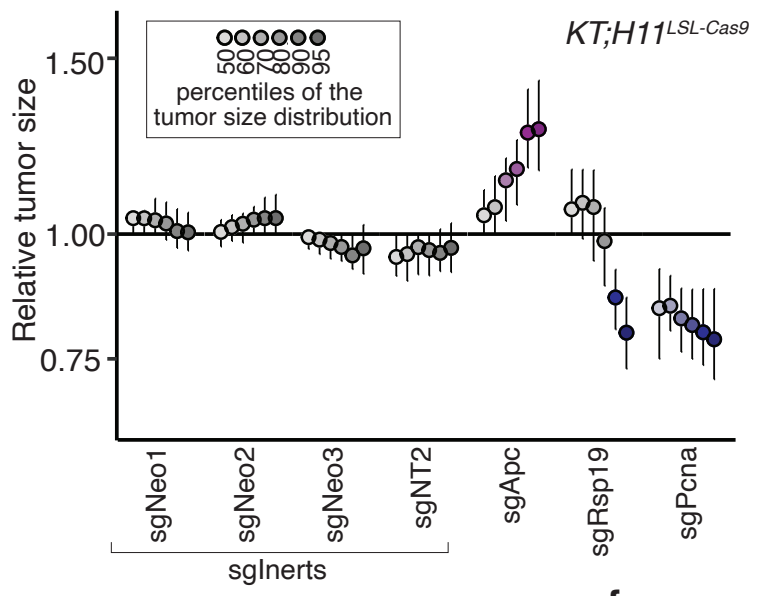

e

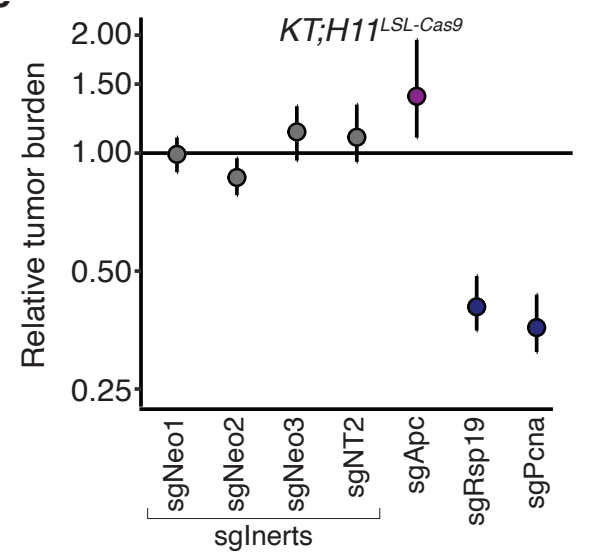

b

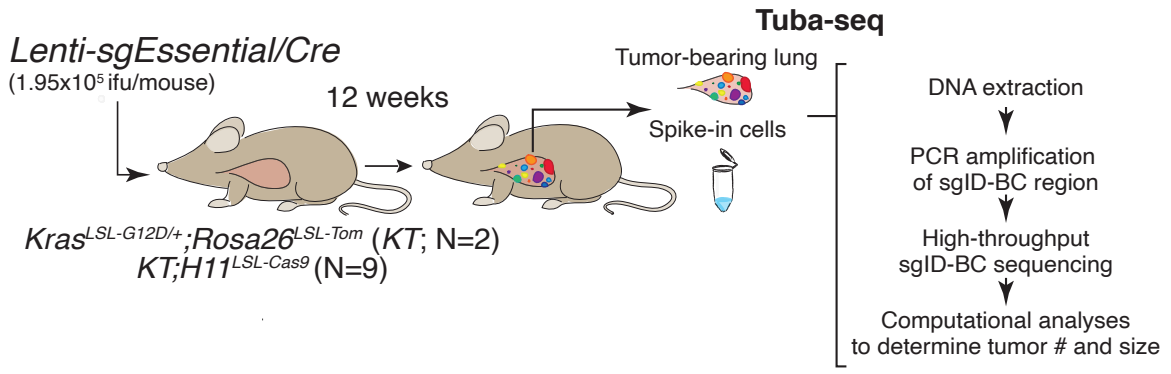

d

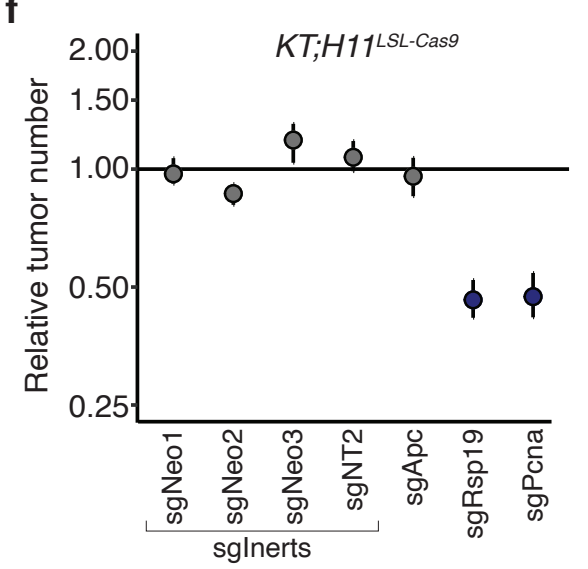

g

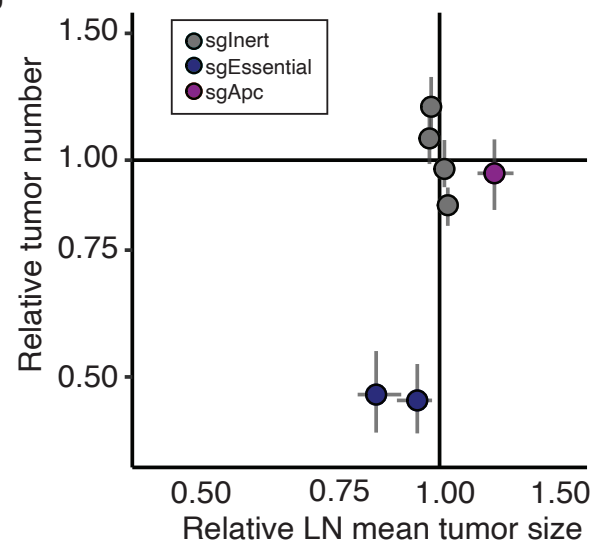

Supplemental Figure 2. Tumor barcoding coupled with barcode sequencing (Tuba-seq) can uncover engineered alterations that reduce tumor number and growth.

a-b. Schematic of the Tuba-seq approach to measure the effects of essential gene inactivation on tumor growth. Lentiviral-sgRNA/Cre vectors with inert sgRNAs (gray) or sgRNAs targeting known essential genes (navy) were diversified with a two component sgID-BC. A vector targeting known tumor suppressor $A p c$ was included as a positive control (a). Tumors were initiated with this barcoded Lenti-sgEssential/Cre pool in KT and KT;H11 LSL-Cas9 mice. Tuba-seq was performed on each tumor-bearing lung 12 weeks after initiation, followed by analyses of sgID-BC sequencing data to characterize the effects of inactivating each gene (b).

c. Tumor sizes at indicated percentiles for each sgRNA relative to the size of sglnert-containing tumors at the corresponding percentiles. Line at $y=1$ indicates no effect relative to sglnert. Error bars indicate $95 \%$ confidence intervals. Percentiles that are signficantly different from sglnert (two-sided FDR-corrected $p<0.05$ ) are in color. Confidence intervals and P-values were calculated by bootstrap resampling. d. The impact of each sgRNA on mean tumor size relative to sglnerts, assuming a log-normal distribution of tumor sizes (LNmean). sgRNAs with two-sided $\mathrm{P}<0.05$ after FDR-adjustment are in bold.

e. The impact of each sgRNA on tumor burden (number of neoplastic cells aggregated across all tumors of a genotype) relative to sglnerts and normalized to the same statistic in $K T$ mice to account for representation of each sgRNA in the viral pool. sg/nerts are in gray and the line at $y=1$ indicates no effect. Error bars indicate $95 \%$ confidence intervals. Relative burdens significantly different from sglnert (two-sided FDR-corrected $\mathrm{p}<0.05$ ) are in color. Confidence intervals and P-values were calculated by bootstrap resampling.

f. The impact of each sgRNA on tumor number relative to sglnerts and normalized to the same statistic in $K T$ mice to account for representation of each sgRNA in the viral pool. sg Inerts are in gray and the line at $y=1$ indicates no effect. Error bars indicate $95 \%$ confidence intervals. Relative tumor numbers significantly different from sglnert (two-sided FDR-corrected $p<0.05$ ) are in color. Confidence intervals and $\mathrm{P}$-values were calculated by bootstrap resampling.

g. The impact of each sgRNA on tumor number plotted against its impact on LNmean tumor size. The lines at $y=1$ and $x=1$ indicate no effect relative to sglnert on tumor number and size, respectively. sgRsp19 and sgPcna cluster in the lower left quadrant near $\mathrm{x}=1$, indicating that targeting essential genes strongly reduces tumor number but only moderately decreases average tumor size. Error bars indicate $95 \%$ confidence intervals calculated by bootstrap resampling. 


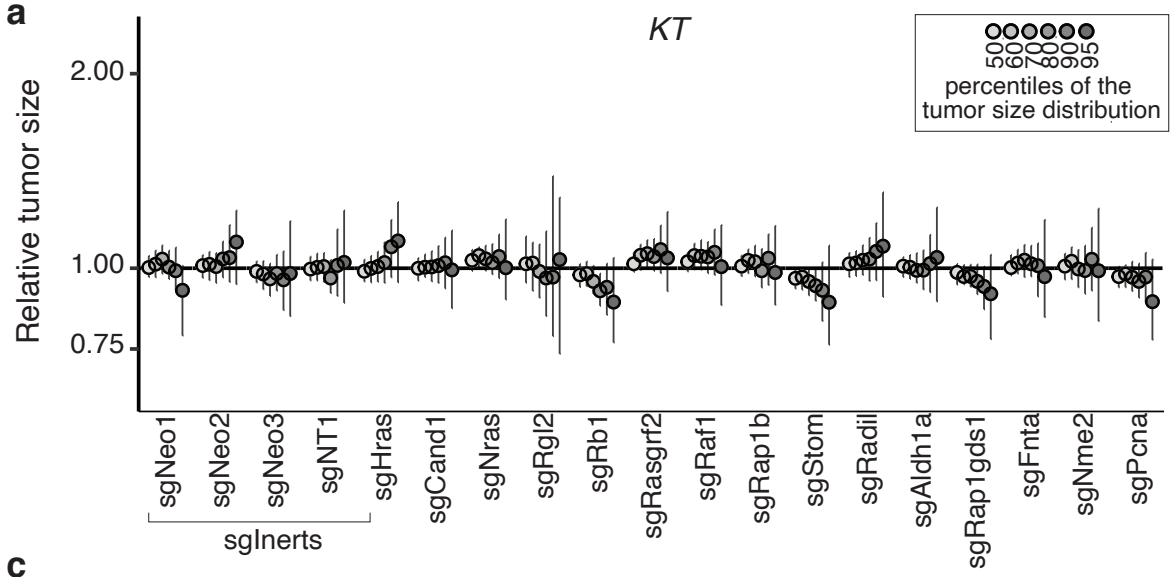

b

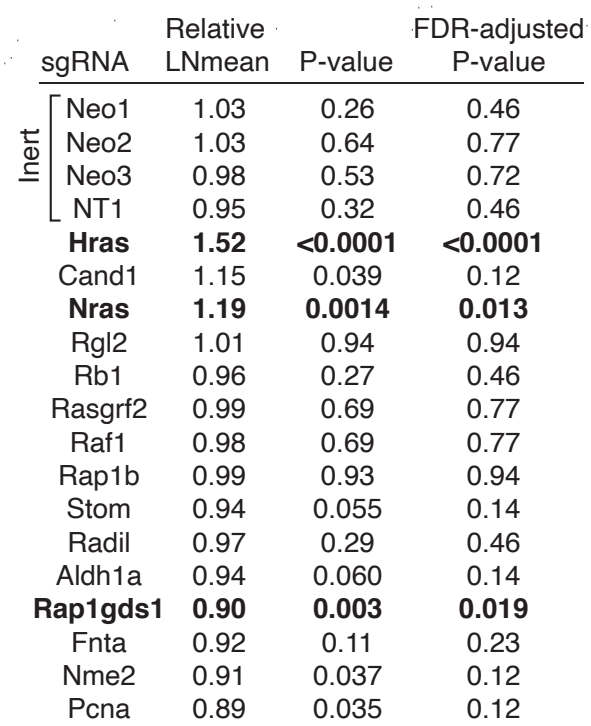
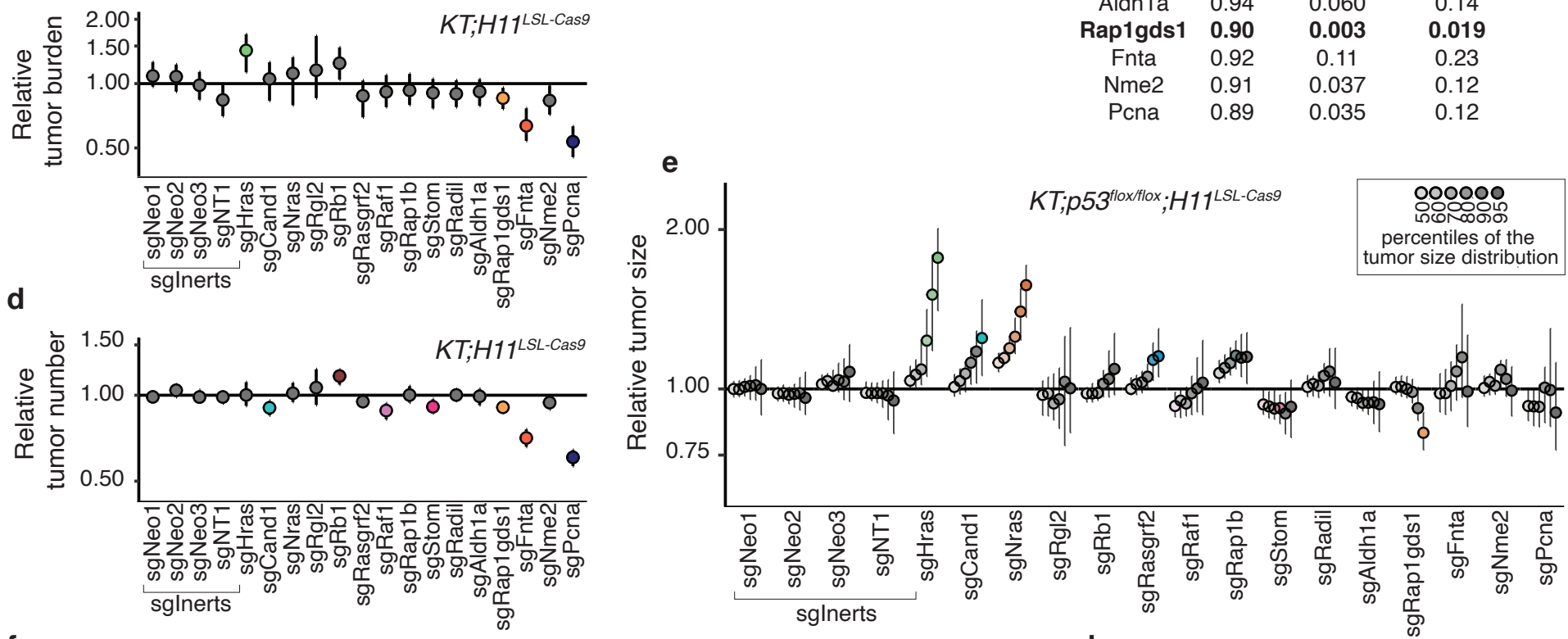

f

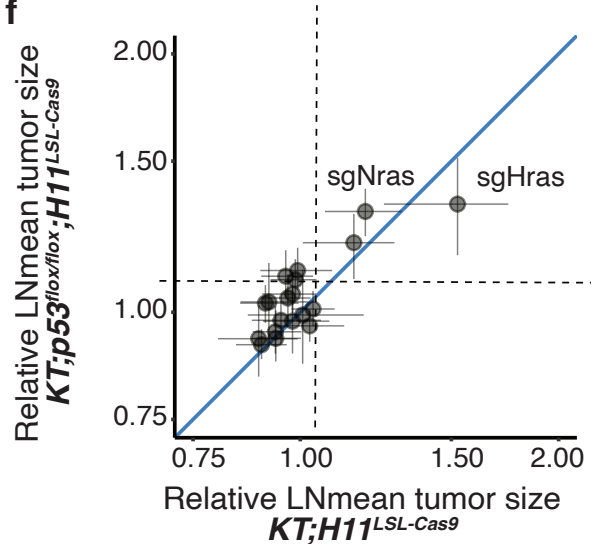

g

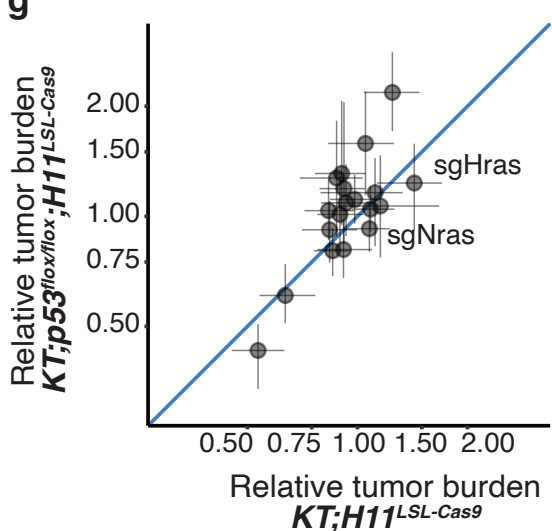

h

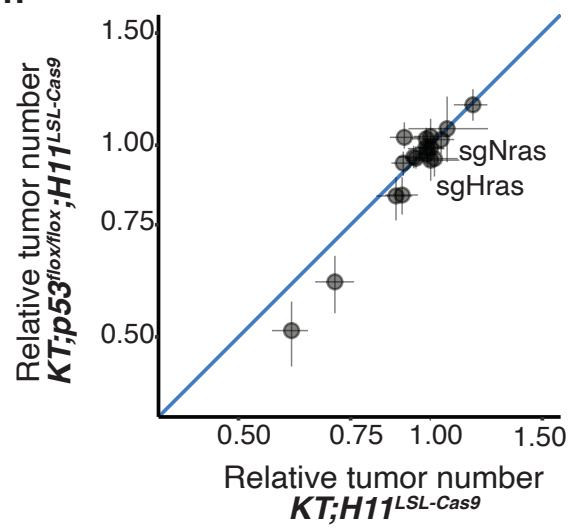

Supplemental Figure 3. Inactivation of KRAS-interacting proteins has similar impacts on tumor growth in p53-proficient and p53-deficient contexts. a. Tumor sizes at indicated percentiles for each sgRNA relative to the size of sglnert-containing tumors at the corresponding percentiles in $K T$ mice. $K T$ mice lack Cas9, thus all sgRNAs are functionally equivalent to sglnerts. Genes are ordered as in Figure 1f. Line at $y=1$ indicates no effect relative to sglnert. Error bars indicate $95 \%$ confidence intervals. Confidence intervals and P-values were calculated by bootstrap resampling. As expected, no percentiles were signficantly different from sgInert (two-sided FDR-adjusted $p<0.05$ ).

b. The impact of each sgRNA on mean tumor size relative to sglnerts in KT;H11LSL-Cas9, assuming a log-normal distribution of tumor sizes (LNmean). sgRNAs with two-sided $\mathrm{P}<0.05$ after FDR-adjustment are in bold. P-values were calculated by bootstrap resampling.

c-d. The impact of each sgRNA on tumor burden (c) and number (d) relative to sglnerts in KT;H11LLL-Cas9 mice, normalized to the corresponding statistic in $K T$ mice to account for representation of each sgRNA in the viral pool. sglnerts are in gray and the line at $\mathrm{y}=1$ indicates no effect. Error bars indicate $95 \%$ confidence intervals. Relative tumor burdens and numbers significantly different from sglnert (two-sided FDR-adjusted $p<0.05)$ are in color. Confidence intervals and P-values were calculated by bootstrap resampling.

e. Tumor sizes at the indicated percentiles for each sgRNA relative to the size of sglnert-containing tumors in KT; $53^{\text {floxfllox; H11 }}{ }^{\text {LSL-Cas9 }}$ mice. Genes are ordered as in Figure 1f. Dashed line indicates no effect relative to sglnert. Error bars indicate 95\% confidence intervals. Percentiles that are signficantly different from sglnert (two-sided FDR-adjusted $\mathrm{p}<0.05$ ) are in color. Confidence intervals and P-values calculated by bootstrap resampling.

$\mathbf{f}-\mathbf{h}$. Comparison of the impact of each sgRNA on relative LNmean tumor size (f), tumor burden (g) and tumor number (h) in KT;H11LSL-Cas9and KT; $553^{\text {floxflox} K-}$

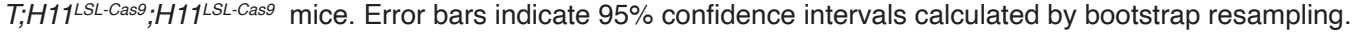


a

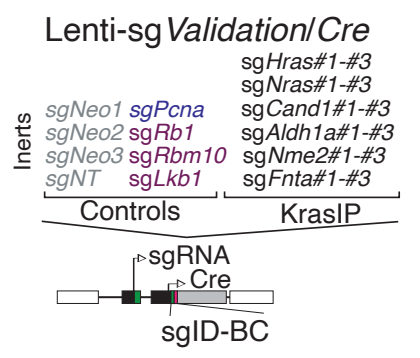

b

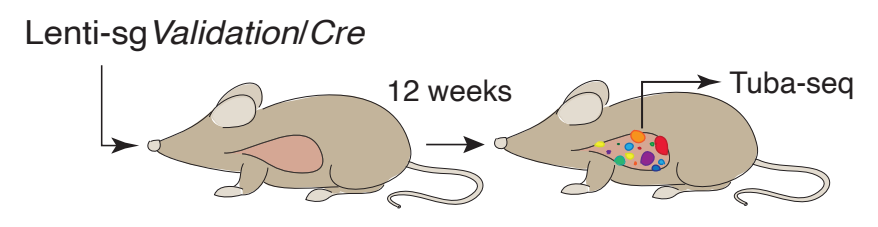

$K T(\mathrm{~N}=5)\left(3 \times 10^{5} \mathrm{ifu}\right)$

$K T ; H 11^{\text {LSL-Cas9 }}(\mathrm{N}=15)\left(3 \times 10^{5} \mathrm{ifu}\right)$
C

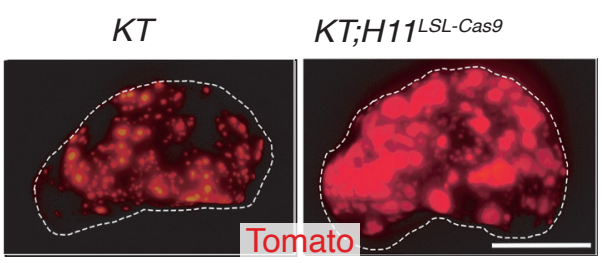

d

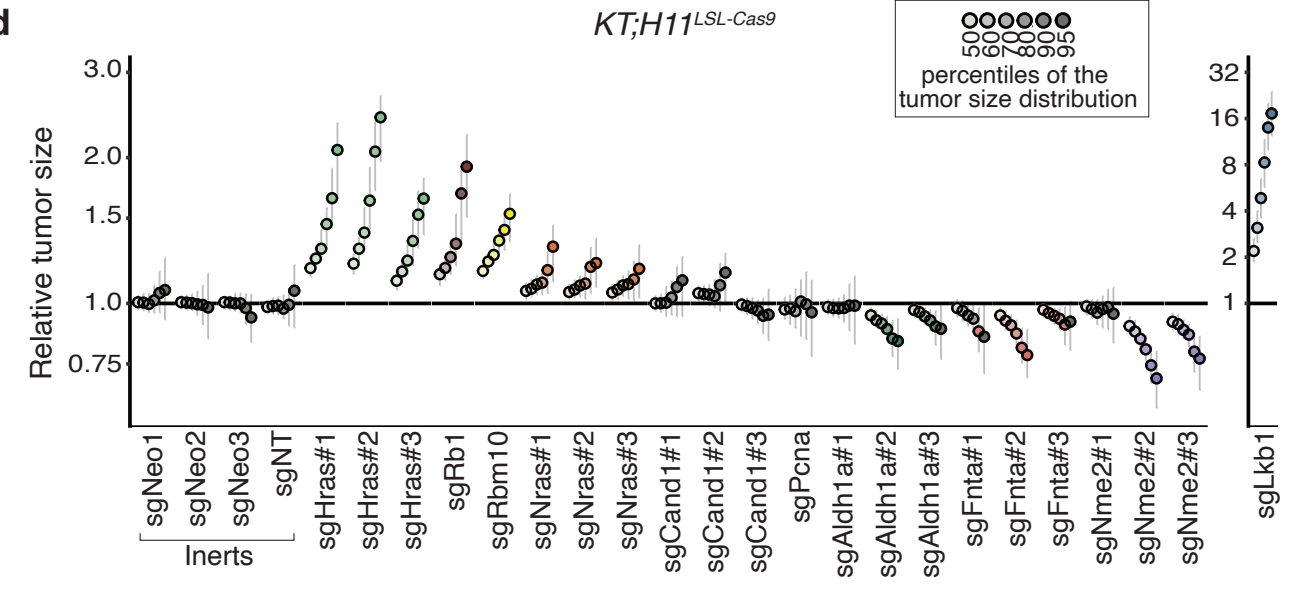

e KT;H11 $1^{\text {LSL-Cas9 }}$

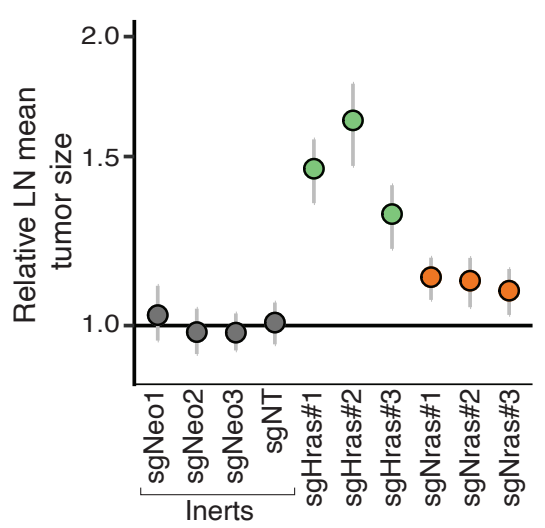

f

Individual vector/mouse Lenti-sg/nert/Cre Lenti-sgHras\#1/Cre $\rightarrow$ Lenti-sgNras\#1/Cre $K T ; H 11^{\text {LSL-Cas9 }}(\mathrm{N}=5 /$ group $)\left(1 \times 10^{5} \mathrm{ifu}\right)$ h

h sg/nert 12 weeks

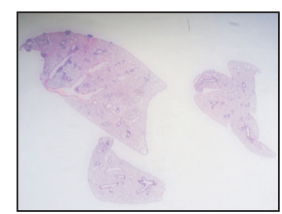

j sg/nert

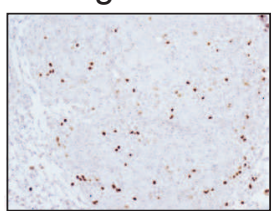

sgHras
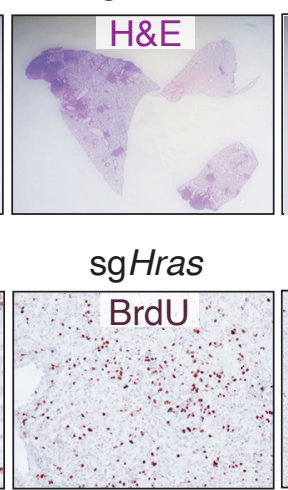

sgNras

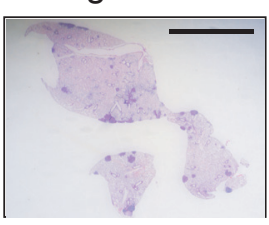

sgNras

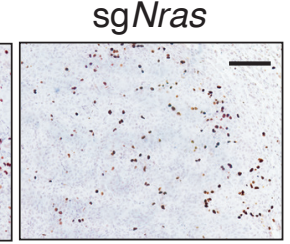

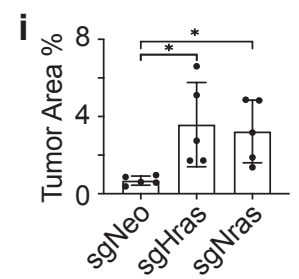

k

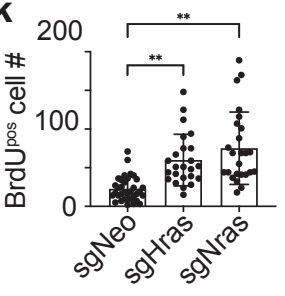

Figure 2. HRAS and NRAS are potent suppressors of KRAS ${ }^{\mathrm{G} 12 \mathrm{D}}$-driven lung cancer growth in vivo

a,b. A pool of barcoded Lenti-sgRNA/Cre vectors (Lenti-sgValidation/Cre) targeting candidate mediators of KRAS-driven lung tumor growth identified in the initial KRAS-interacting protein Tuba-seq screen was used to initiate tumors in validation cohorts of $K T$ and $K T ; H 11^{L L L-C a s 9}$ mice. This lentiviral pool includes four Inert sgRNAs, as well as sgRNAs targeting $L k b 1, R b 1$, and $R b m 10$ as tumor suppressor controls. Each candidate gene from the initial screen is targeted with three sgRNAs. Tumors were initiated throuh intratracheal delivery of Lenti-sgValidation/Cre, and Tuba-seq was performed on each tumor-bearing lung 12 weeks after initiation, followed by analyses of sgID-BC sequencing data to characterize the effects of inactivating each gene (b).

c. Fluorescence images of representative lung lobes 12 weeks after tumor initiation. Scale bars $=5 \mathrm{~mm}$. Lung lobes are outlined with a white dashed line.

d. Tumor sizes at indicated percentiles for each sgRNA relative to the size of sglnert-containing tumors at the corresponding percentiles in $K T ; H 11^{L L L-C a s 9}$ mice. Genes are ordered by $95^{\text {th }}$ percentile tumor size, with sglnerts on the left. Note that sgLkb1 is plotted on a separate scale to facilitate visualization of sgRNAs with lesser magnitudes of effect. Dashed line indicates no effect relative to sglnert. Error bars indicate $95 \%$ confidence intervals. 95\% confidence intervals and P-values were calculated by bootstrap resampling. Percentiles that are signficantly different from sgInert $(2$-sided FDR-adjusted $p<0.05)$ are in color.

e. Targeting Hras and Nras significantly increases mean tumor size relative to sglnerts, assuming a log-normal distribution of tumor sizes (LNmean). Error bars indicate 95\% confidence intervals calculated by bootstrap resampling.

f. Schematic of tumor initiation with individual Lenti-sgRNA/Cre vectors. Mouse number and titer of the lentiviral vectors are indicated.

g. Representative fluorescence images of lungs from KT;H11 LSL-Cas9 mice after tumor initiation with Lenti-sgRNA/Cre vectors as indicated. Scale bar $=5 \mathrm{~mm}$.

h. Representative H\&E images of lungs from KT;H11 LSL-Cas9 mice after tumor initiation with Lenti-sgRNA/Cre vectors as indicated. Tumor area (percentage of total lung area) from each mouse is shown as Mean \pm SD. ${ }^{*}$ : $p<0.05$; Scale bar $=5 \mathrm{~mm}$.

i. Tumor burden in KTC mice with tumors initiated with Lenti-sgRNA/Cre vectors as indicated. Each dot represents relative tumor area (percentage of total lung area) from one mouse. *: $p<0.05$

j. Representative BrdU staining images of lungs from KT;H11 LSL-Cas9 mice after tumor initiation with Lenti-sgRNA/Cre vectors as indicated. Number of Brdu+ cells per field is shown as Mean \pm SD. ${ }^{* *}: p<0.01$; Scale bar $=100 \mu \mathrm{m}$.

k. Quantification of proliferation cells in KTC mice with tumors initiated with Lenti-sgRNA/Cre vectors as indicated. Each dot represents a tumor. ${ }^{* *}: p<0.01$ 
a

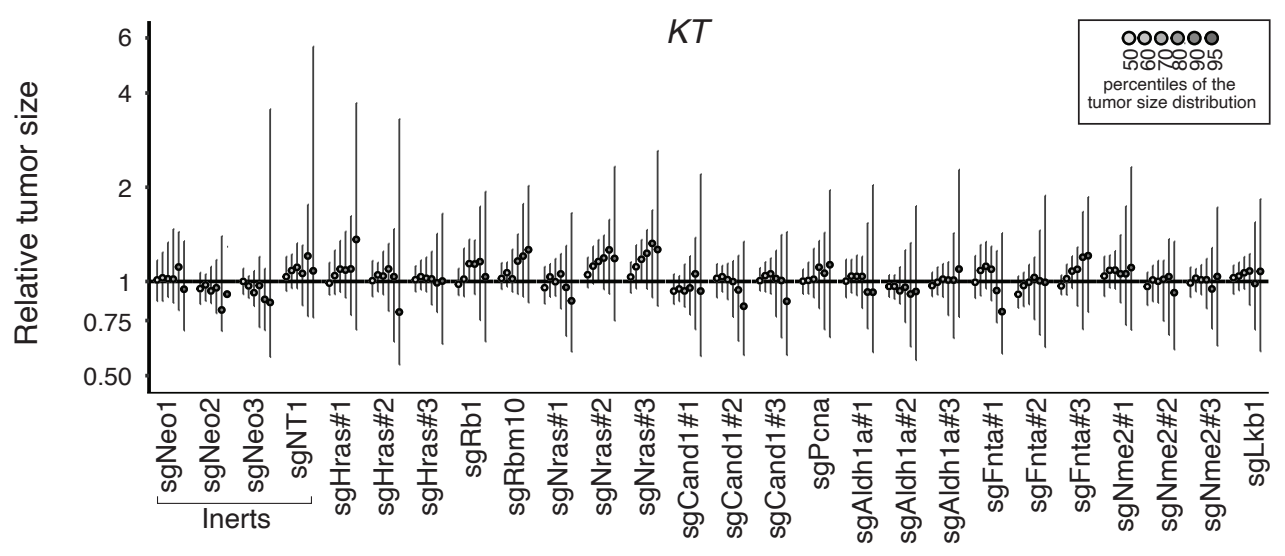

b

\begin{tabular}{|c|c|c|c|}
\hline sgRNA & $\begin{array}{l}\text { Relative } \\
\text { LNmean }\end{array}$ & $P$-value & $\begin{array}{c}\text { FDR-adjusted } \\
\text { P-value }\end{array}$ \\
\hline 「Neo1 & 1.03 & 0.45 & 0.51 \\
\hline $\mathrm{Neo} 2$ & 0.98 & 0.57 & 0.59 \\
\hline $\mathrm{Neo3}$ & 0.98 & 0.43 & 0.51 \\
\hline NT1 & 1.01 & 0.82 & 0.82 \\
\hline Lkb1 & 8.85 & $<0.001$ & $<0.001$ \\
\hline Hras\#1 & 1.31 & $<0.001$ & $<0.001$ \\
\hline Hras\#2 & 1.46 & $<0.001$ & $<0.001$ \\
\hline Hras\#3 & 1.64 & $<0.001$ & $<0.001$ \\
\hline Rb1 & 1.41 & $<0.001$ & $<0.001$ \\
\hline Rbm10 & 1.31 & $<0.001$ & $<0.001$ \\
\hline Nras\#1 & 1.09 & 0.0050 & 0.0090 \\
\hline Nras\#2 & 1.11 & 0.0014 & 0.0028 \\
\hline Nras\#3 & 1.12 & $<0.001$ & $<0.001$ \\
\hline Cand1\#1 & 1.06 & 0.038 & 0.059 \\
\hline Cand1\#2 & 0.97 & 0.16 & 0.23 \\
\hline Cand1\#3 & 1.03 & 0.48 & 0.52 \\
\hline Pcna & 0.96 & 0.38 & 0.46 \\
\hline Aldh1a\#1 & 0.96 & 0.25 & 0.34 \\
\hline Aldh1a\#2 & 0.93 & 0.0052 & 0.0090 \\
\hline Aldh1a\#3 & 0.90 & $<0.001$ & $<0.001$ \\
\hline Fnta\#1 & 0.93 & $<0.001$ & $<0.001$ \\
\hline Fnta\#2 & 0.92 & 0.017 & 0.027 \\
\hline Fnta\#3 & 0.87 & $<0.001$ & $<0.001$ \\
\hline Nme2\#1 & 0.82 & $<0.001$ & $<0.001$ \\
\hline Nme2\#2 & 0.86 & $<0.001$ & $<0.001$ \\
\hline Nme2\#3 & 0.98 & 0.33 & 0.43 \\
\hline
\end{tabular}

C

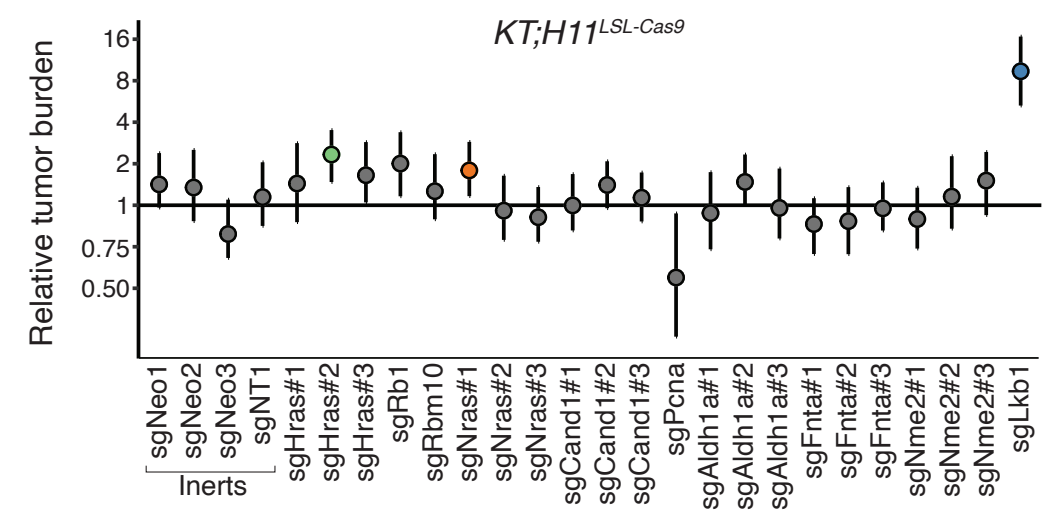

d

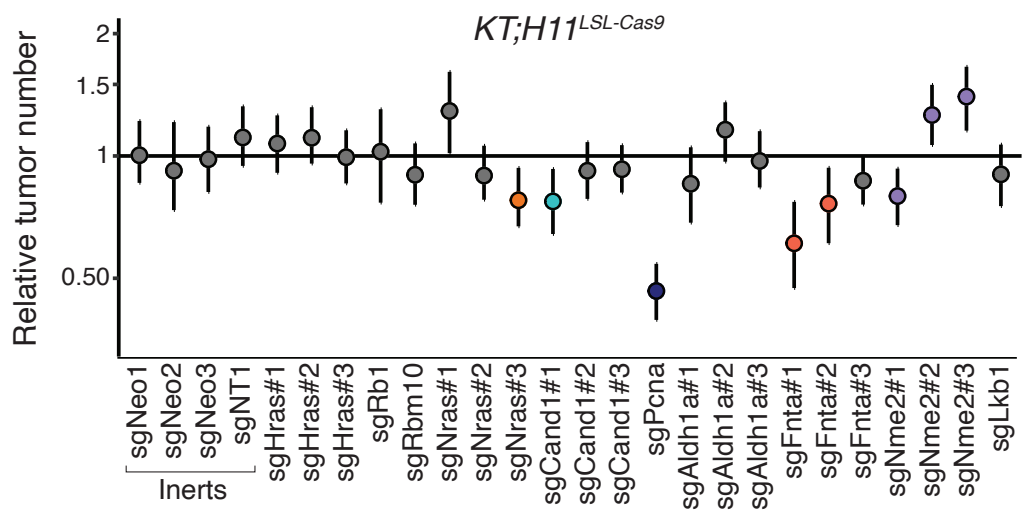

Supplemental Figure 4. Top candidate KRAS-interacting proteins from initial Tuba-seq screen impact multiple metrics of tumor growth in validation cohort.

a.Tumor sizes at indicated percentiles for each sgRNA relative to the size of sglnert-containing tumors at the corresponding percentiles in $K T$ mice. KT mice lack Cas9, thus all sgRNAs are functionally equivalent to sglnerts. Genes are ordered as in Figure $2 \mathrm{~d}$, but note the change in axis scaling. Line at $\mathrm{y}=1$ indicates no effect relative to sglnerts. Error bars indicate $95 \%$ confidence intervals. Confidence intervals and P-values were calculated by bootstrap resampling. As expected, no percentiles were signficantly different from sglnert (FDR-adjusted $p<0.05$ ).

b. The impact of each sgRNA on mean tumor size relative to sglnerts, assuming a log-normal distribution of tumor sizes (LNmean). Two-sided P-values were calculated by bootstrap resampling. sgRNAs with $\mathrm{P}<0.05$ after FDR-adjustment are in bold. Note that this data for the sglnerts, sgHras\#1-3 and sgNras\#1-3 is also plotted in Figure 2e.

c. The impact of each sgRNA on tumor burden relative to sglnerts in KT;H11 LSL-Cas9 mice, normalized to the corresponding statistic in $K T$ mice to account for representation of each sgRNA in the viral pool. sglnerts are in gray and the line at $y=1$ indicates no effect. Error bars indicate $95 \%$ confidence intervals. Relative tumor burdens significantly different from sglnert (two-sided FDR-adjusted p<0.05) are in color. Confidence intervals and $\mathrm{P}$-values were calculated by bootstrap resampling.

d. The impact of each sgRNA on tumor number relative to sglnerts in KT;H11 LSL-Cas9 mice, normalized to the corresponding statistic in KT mice to account for representation of each sgRNA in the viral pool. sgInerts are in gray and the line at $\mathrm{y}=1$ indicates no effect. Error bars indicate $95 \%$ confidence intervals. Relative tumor numbers significantly different from sglnert (two-sided FDR-adjusted p<0.05) are in color. Confidence intervals and $\mathrm{P}$-values were calculated by bootstrap resampling. 
a

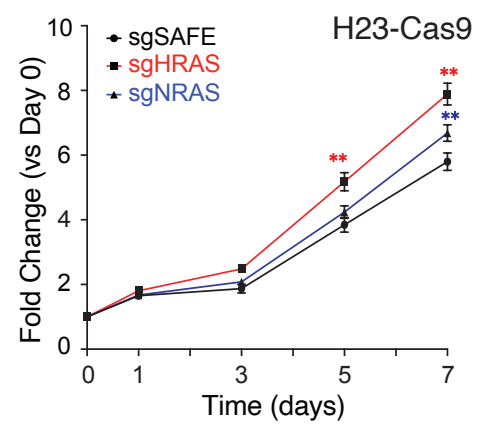

C

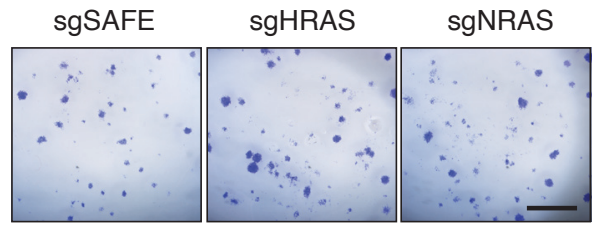

b
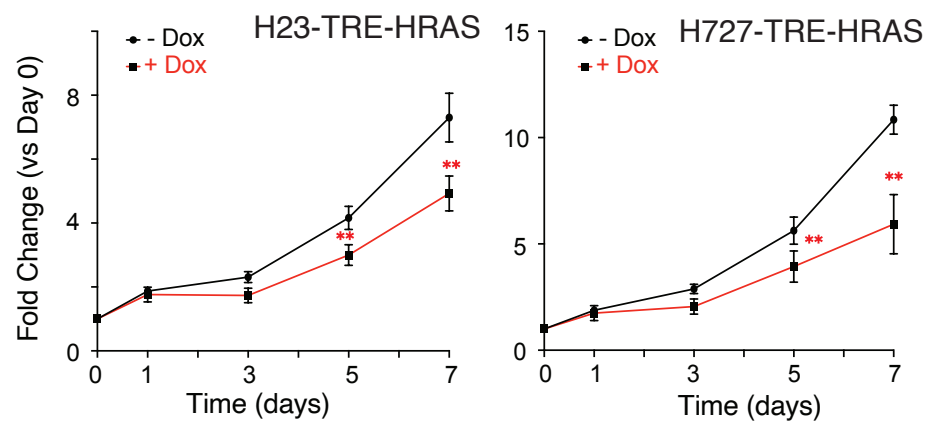

e
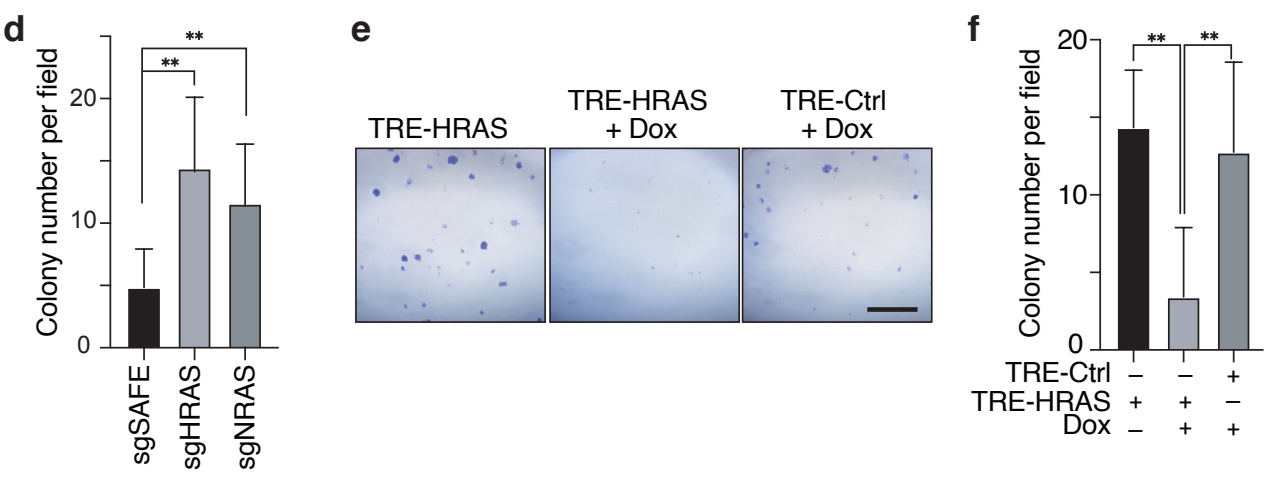

g

H23-Cas9-sgSAFE H23-Cas9-sgHRAS H23-Cas9-sgNRAS

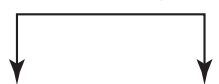

SubQ Intravenous (iv) ( $2 \times 10^{6}$ cells) $\left(5 \times 10^{5}\right.$ cells)

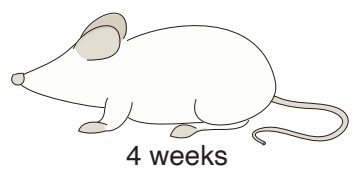

$\stackrel{N}{N \text { NSG }} \stackrel{N}{N S G}$ h

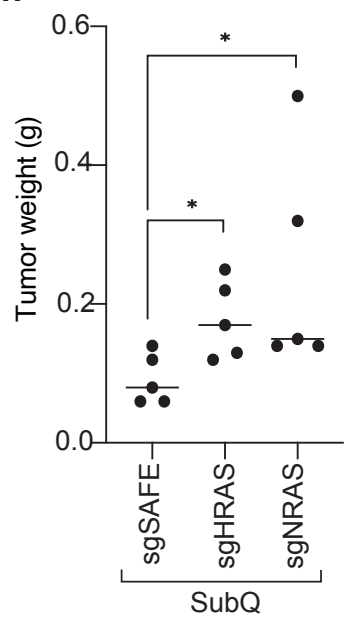

i

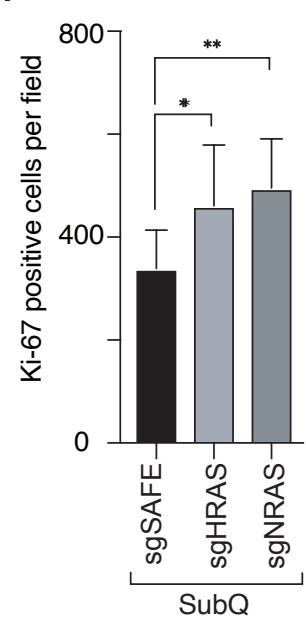

j

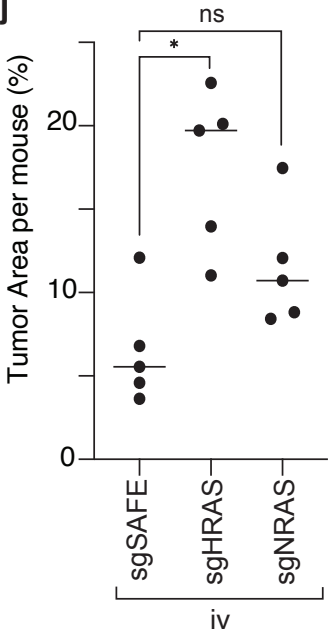

k

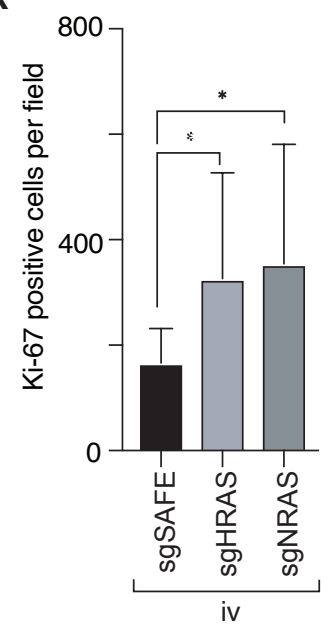

Figure 3. Wildtype HRAS or NRAS constrain the growth of human KRAS-driven cancer cell lines.

a. Inactivation of wild type HRAS or NRAS increases growth of KRAS-mutant H23 (G12C) and H727 (G12V) cells. Wildtype (sgSAFE) or HRAS- or NRAS-knockout cells were seeded in 96 well plates and cultured under limited serum (1\%). Cell numbers were measured via CCK8 assay. Points are Mean \pm SD of 12 wells normalized to Day $0 .{ }^{* *}: p<0.01$

b. Re-expression of wild type HRAS suppresses proliferation of HRAS-null H23 and H727 cells. TRE-HRAS cells were seeded in 96 well plates and cultured under limited serum (1\%) with or without $50 \mathrm{ng} / \mathrm{ml}$ Doxycycline (Dox) and cell numbers were measured via CCK8 assay. Points are Mean \pm SD of 12 wells normalized to Day $0 .{ }^{* *}: p<0.01$

c-d. Inactivation of HRAS or NRAS increases H23 colony formation. Wildtype (sgSAFE), HRAS-knockout (sgHRAS), or NRAS-knockout (sgNRAS) H23 cells were seeded at 1000 cells/well in 6-well plates and grown for two weeks. Cells were stained with crystal violet. c.

Representative images. Scale bar $=5 \mathrm{~mm}$. d. Mean \pm SD of colony number of 12 fields. ${ }^{* *}: p<0.01$

e-f. Re-expression of wild type HRAS suppresses HRAS-null H23 cell colony formation. TRE-Ctrl or TRE-HRAS H23 cells were seeded at 1000 cells/well in 6-well plates and grown with or without $50 \mathrm{ng} / \mathrm{ml}$ Doxycycline (Dox) for two weeks. Cells were stained with crystal violet. e. Representative images. Scale bar $=5 \mathrm{~mm}$. $\mathbf{f}$. Mean $\pm S D$ of colony number of 12 fields. ${ }^{* *}: p<0.01$

g-k. Inactivation of wild type HRAS or NRAS increases $\mathrm{H} 23$ cell growth after transplantation. g. Schematic of tumor initiation with subcutaneous (SubQ) or intravenous (IV) transplantation of H23 cells with inactivation of HRAS or NRAS in NSG mice. Mouse number, cell number, and tumor growth time after transplantation are indicated. $\mathbf{h}$. Tumor weight from SubQ transplantation of indicated cells.

Each dot represents a mouse. Mean value was shown. i. Ki67 ${ }^{\text {pos }}$ cell number in tumor section from SubQ transplantation of indicated cells was shown as Mean \pm SD value of 20 view fields. $\mathbf{j}$. Tumor area (percentage of h-mitochondria ${ }^{\text {pos }}$ area) from IV transplantation of indicated cells. Each dot represents a tumor. Mean value was shown. k. Ki67 ${ }^{\text {pos }}$ cell number in tumor section from IV transplantation of indicated cells is shown as Mean $\pm S D$ value of 20 view fields $(200 x) .{ }^{*}: p<0.05 ;{ }^{* *}: p<0.01$; ns: not significant. 
a

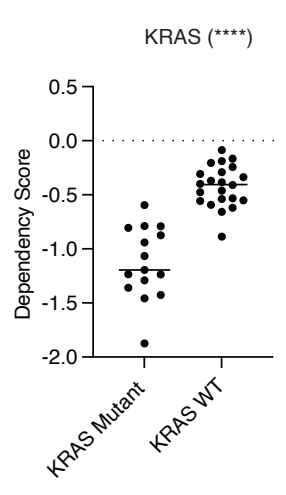

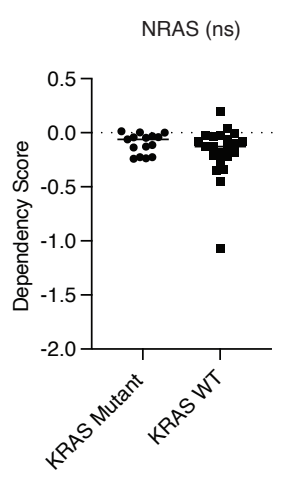

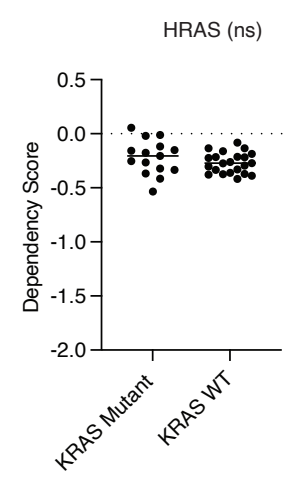

b

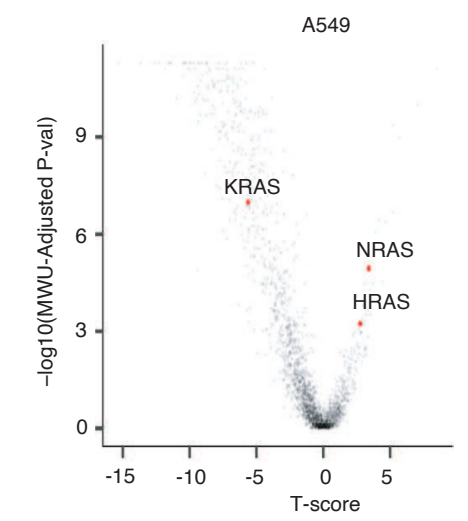

c
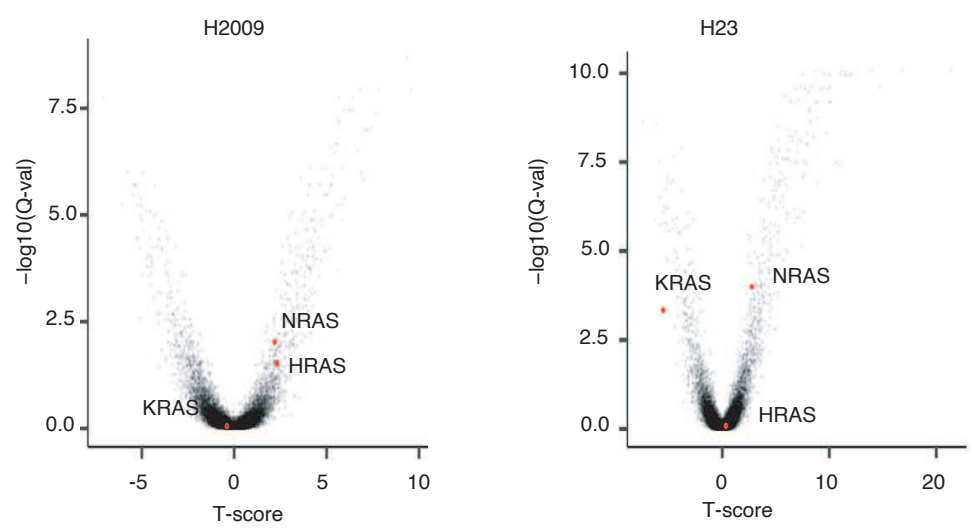

H1975

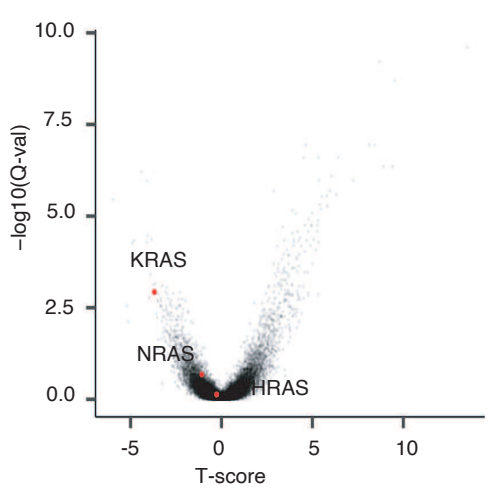

Supplemental Figure 5. Dependency of human LUAD cell lines on RAS family members.

a. Comparison of RAS family member dependency scores between KRAS mutant and KRAS wildtype human LUAD cell lines. ${ }^{* * * *}(p<0.0001)$, ns (not-significant). (Data source: DepMap)

b. Volcano plot showing the effects of RAS gene knockouts in A549 cells. The T-score represents the normalized effect of multiple sgRNAs targeting a gene. A positive T-score indicates a tumor suppressive effect. The effects of each gene relative to SAFE sgRNAs were tested via Mann-Whitney U (MWU) test, corrected via Benjamini-Hochberg procedure and shown as -log10(MWU-Adjusted P-val). (Data source: Marcus Robert Kelly, Kaja Kostyrko, Kyuho Han, et al. 2020)

c. Volcano plot showing effects of RAS gene knockouts in KRAS-mutant human LUAD cells (left: H2009, mid: H23, right: $\mathrm{H} 1975)$ in 3D culture. The T-score represents the normalized effect of multiple sgRNAs targeting a gene. A positive T-score indicates a tumor suppressive effect. The effects of each gene relative to SAFE sgRNAs were tested via two-side t-test, corrected via Benjamini-Hochberg procedure and shown as -log10(Q-val). (Data source: Kyuho Han, et al. 2020) 
a

$$
\text { sgSAFE }
$$

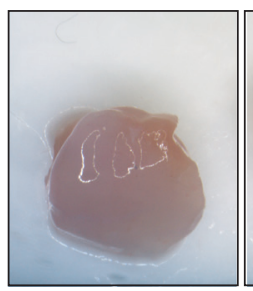

b

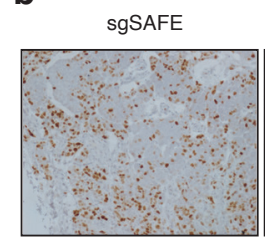

sgHRAS

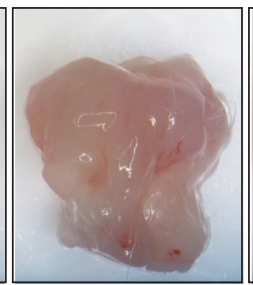

SgHRAS

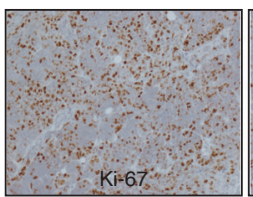

SgNRAS

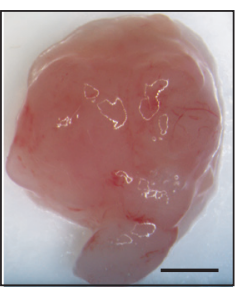

SgNRAS

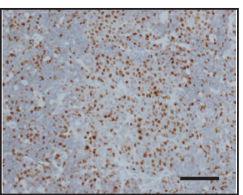

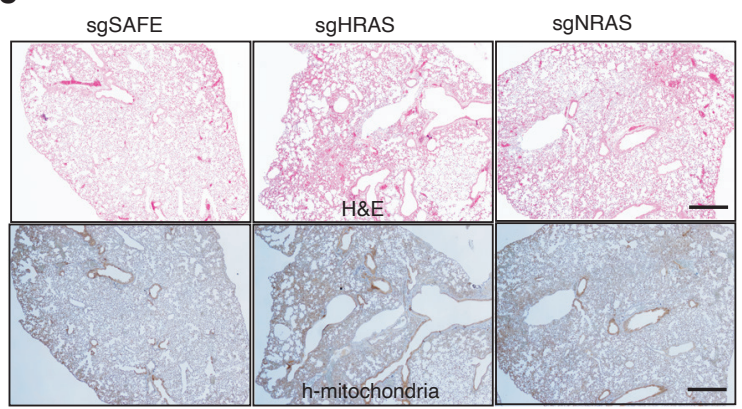

d

sgSAFE

sgHRAS

sgNRAS

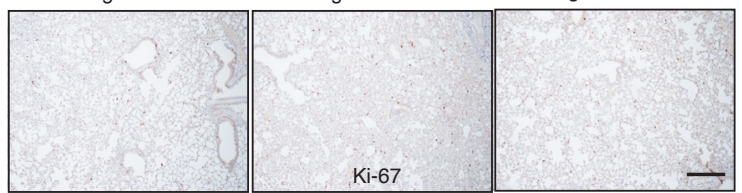

Supplemental Figure 6. Inactivation of wild type HRAS or NRAS increases H23 cell growth after transplantation.

a. Representative image of subcutaneous tumor size four weeks after transplantation with H23 cells as indicated. Quantification was shown in Figure $3 \mathrm{~h}$. Scale bar: $2 \mathrm{~mm}$

b. Representative image of Ki67 staining from subcutaneous tumor four weeks after transplantation with $\mathrm{H} 23$ cells as indicated. Quantification was shown in Figure 3i. Scale bar: $100 \mu \mathrm{m}$

c. Representative image of HE (upper) and human mitochondria (lower) staining from lung tumor four weeks after intravenous transplantation with $\mathrm{H} 23$ cells as indicated. Quantification was shown in Figure 3j. Scale bar: $500 \mu \mathrm{m}$

d. Representative image of Ki67 staining from lung tumor four weeks after intravenous transplantation with $\mathrm{H} 23$ cells as indicated. Quantification was shown in Figure 3k. Scale bar: $200 \mu \mathrm{m}$ 
a

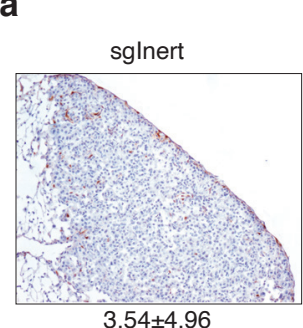

C

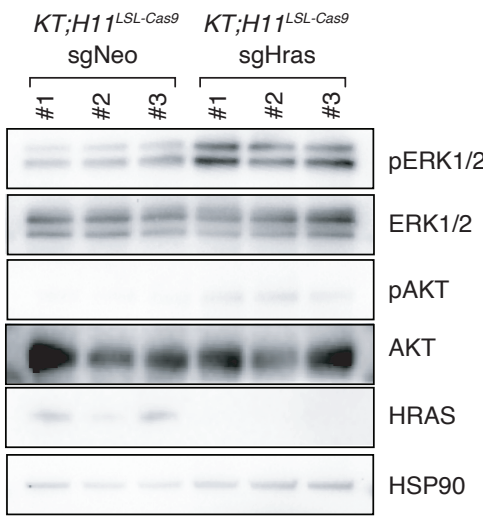

f H23-sgHRAS- HOP62-sgHRAS-

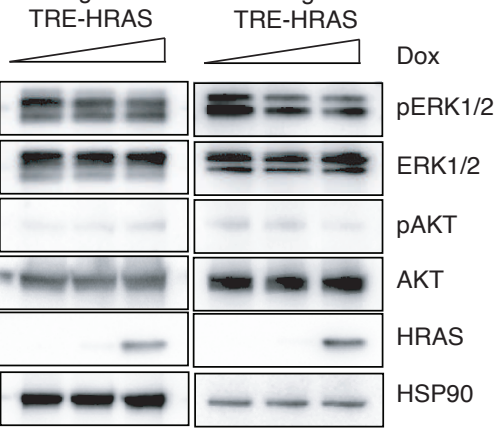

$K T ; H 11^{\text {LLL-Cas9 }}$

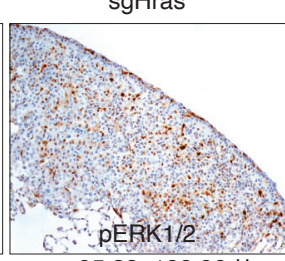

$95.33 \pm 188.96$ **

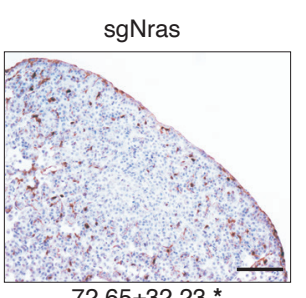

$72.65 \pm 32.23$ *

b

d

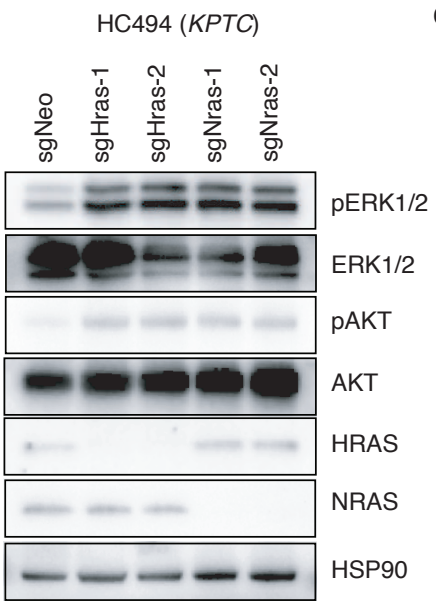

g

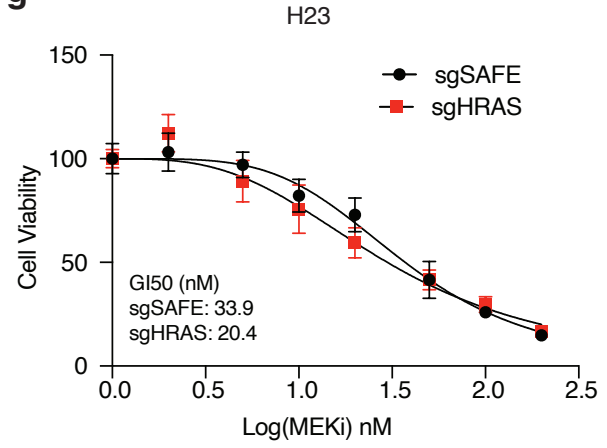

sgSAFE

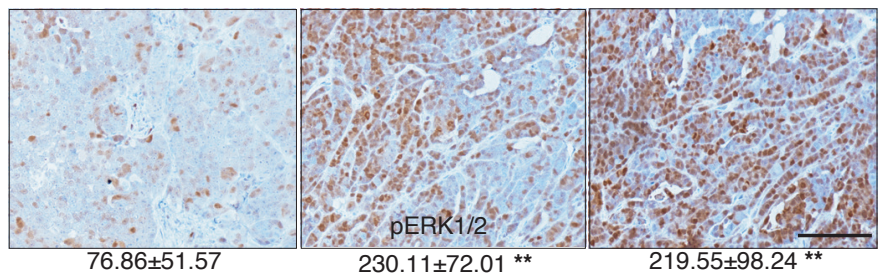

e

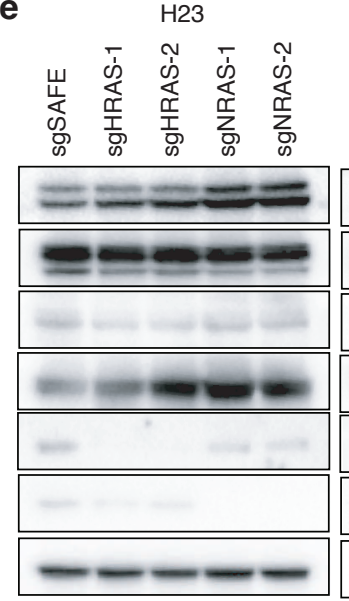

HOP62

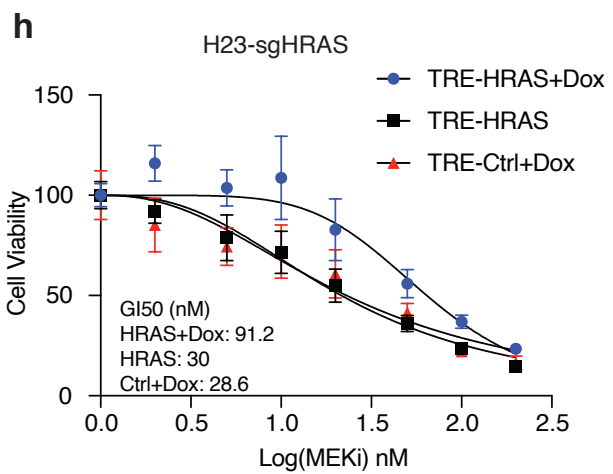

Figure 4. Wildtype RAS paralogs suppress RAS signaling

a. Representative image of pERK staining in KTC mice with tumors initiated with Lenti-sgRNA/Cre vectors as indicated. Quantification of pERK ${ }^{\text {pos }}$ cells per tumor was shown as Mean \pm SD of 20 tumors. ${ }^{*}$ : $p<0.05 ;{ }^{* *}$ : $p<0.01$; Scale bar: $100 \mu \mathrm{m}$

b. Representative image of $p E R K$ staining in subcutaneous tumor transplantated with $\mathrm{H} 23$ cells as indicated. Quantification of $p E R K^{\text {pos }}$ cells per field was shown as Mean \pm SD of 20 fields. ${ }^{*}: p<0.01$; Scale bar: $100 \mu \mathrm{m}$. HSP90 shows loading.

c. Western blot analysis of sorted cancer cells from KT;H11 LSL-Cas9 mice transduced with Lenti-sgRNA/Cre vectors as indicated. Multiple tumors were pooled and Tomato-positive cancer cells were sorted prior to and protein extraction. HSP90 shows loading.

d. Western blot analysis of murine lung adenocarcinoma cell line that was transduced with Lenti-sgRNA vectors as indicated and selected with puromycin to generate stable knockout cell lines. Wildtype cells (sgNeo) or HRAS- or NRAS-knockout cells (sgHras, sgNras) were cultured under limited serum (1\%) for 2 days before protein extraction. HSP90 shows loading.

e. Western blot analysis of cultured human lung adenocarcinoma cell lines transduced with Lenti-sgRNA vectors as indicated and selected with puromycin to generate stable knockout cell lines. Wildtype cell (sgSAFE) or HRAS- or NRAS-knockout cells (sgHRAS, sgNRAS) were cultured under limited serum (1\%) for 2 days before protein extraction. HSP90 shows loading.

f. Western blot analysis of human lung adenocarcinoma cell lines re-expression HRAS (TRE-HRAS) under Doxycycline (Dox) treatment. HRAS-null cells were generated as described in Figure 3a. HRAS-null cells were re-transduced with lentiviral vector expressing TRE-HRAS at high MOI (>5) to generate stable HRAS re-expression cells (sgHRAS-TRE-HRAS). To re-express HRAS, cells were treated with 0,1 , or $2 \mathrm{ng} / \mathrm{ml}$ Dox and cultured under limited serum (1\%) for 2 days before protein extraction. HSP90 shows loading.

g. Comparison of Gl50 values to MEK inhibitors trametinib among wildtype and HRAS-null H23 cells under treatment of indicated dose of trametinib for four days. Cell number were measured via CCK8 assay and normalized to cells treated with vehicle. Each data point was shown as Mean \pm SD of 12 wells.

h. Comparison of GI50 values to MEK inhibitors trametinib among HRAS-null H23 cells (H23-sgHRAS) re-expressing HRAS in presence (HRAS+Dox) or absence (HRAS) of Doxycycline plus indicated dose of trametinib for four days. Cell number were measured via CCK8 assay and normalized to cells treated with vehicle. Each data point was shown as Mean \pm SD of 12 wells. 
a

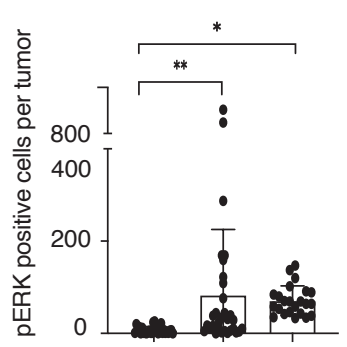

b
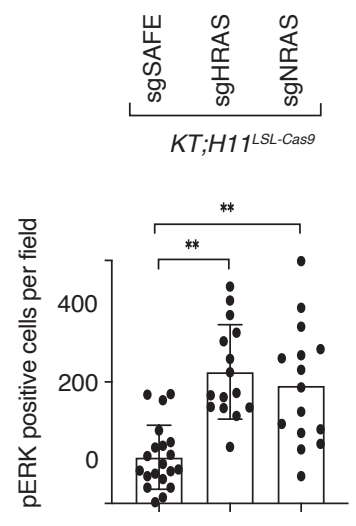

e

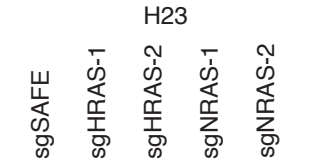

55

35

55

35

75

55

75

55

35

15

35

180

75
C

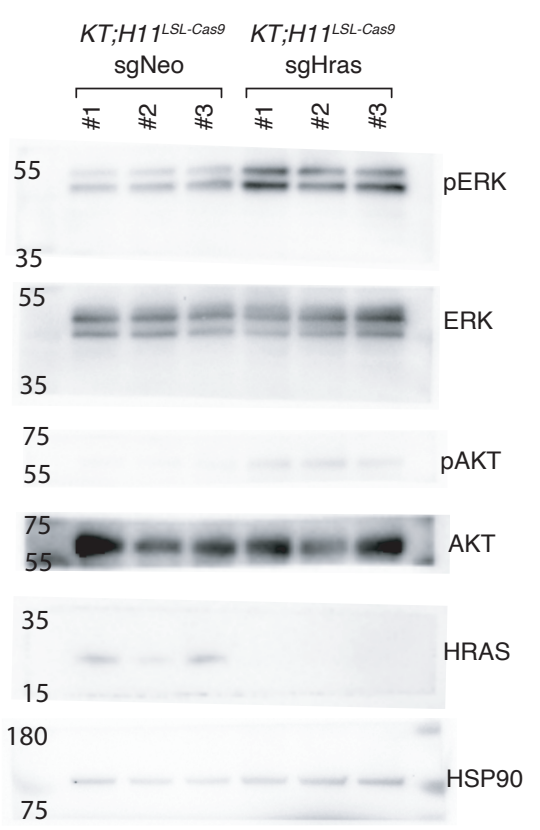

d

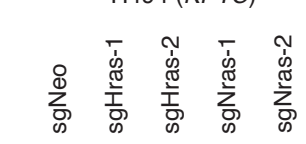

55

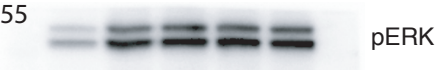

35

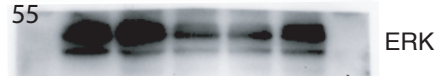

35

75 PAKT

55

75000 AKT

35
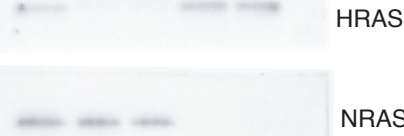

15

$180+--$ HSP9O

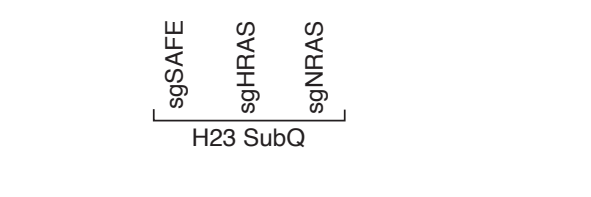

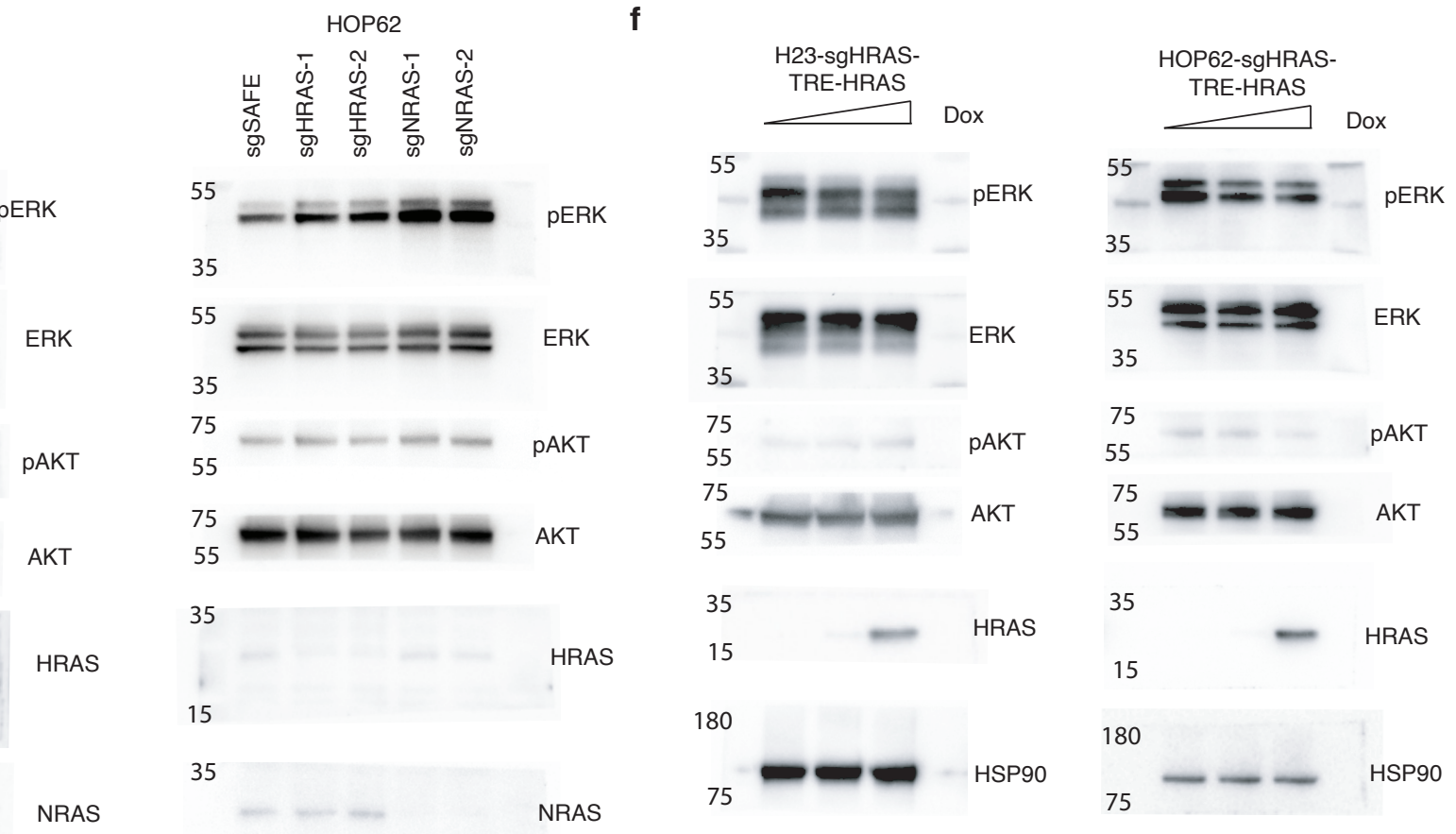

Supplemental Figure 7. Wildtype RAS paralogs finetune RAS signaling.

a. Quantification of pERK ${ }^{\text {pos }}$ cells in KT;H11LLL-Cas9 mice with tumors initiated with Lenti-sgRNA/Cre vectors as indicated in Figure 4a. Each dot represents a tumor. ${ }^{*}: p<0.05 ; * *: p<0.01$

b. Quantification of $p E R K^{p o s}$ cells per field of indicated cells from Figure $4 b$. Each dot represents a view field. ${ }^{* *}: p<0.01$

c-f. Raw images for western blots from Figure $\mathbf{4 c - f}$. HRAS expression on Figure $\mathbf{4 f}$ were detected using same lysis on a different gel with increased loading. 
a

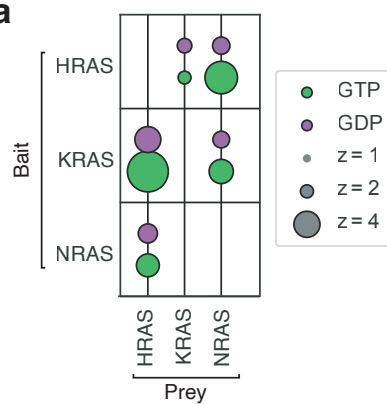

d

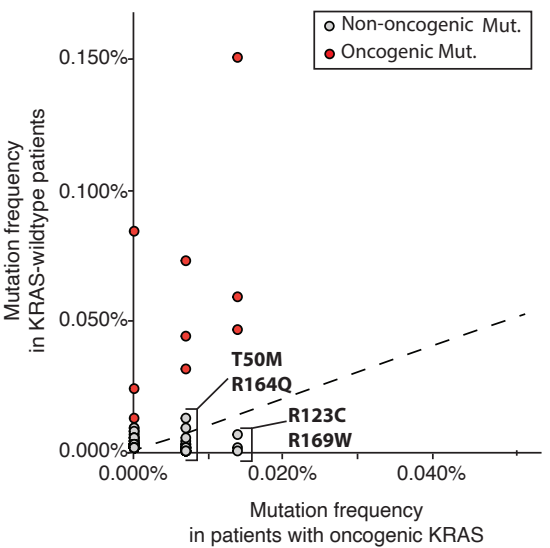

g

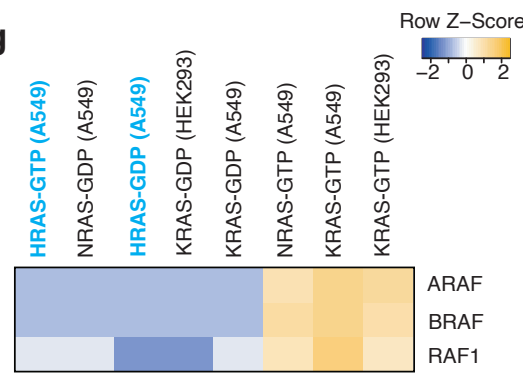

b
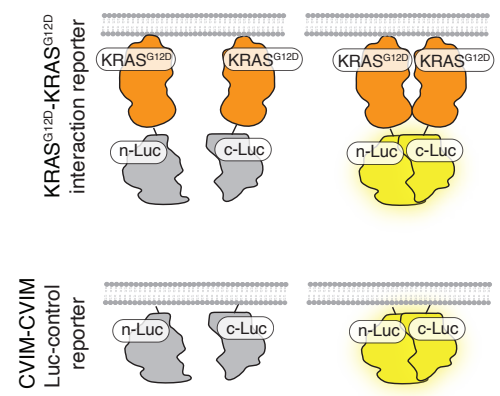

e

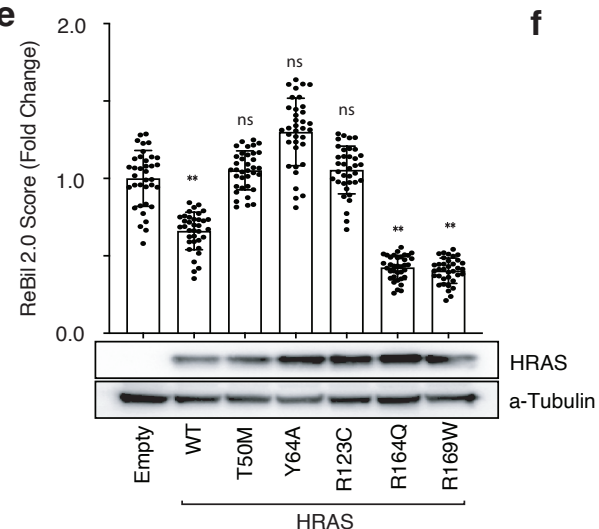

h

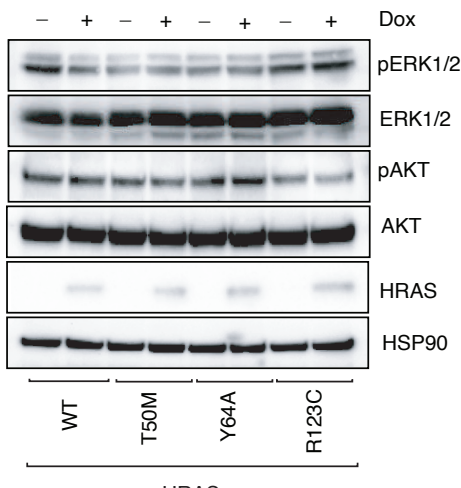

f

i
C

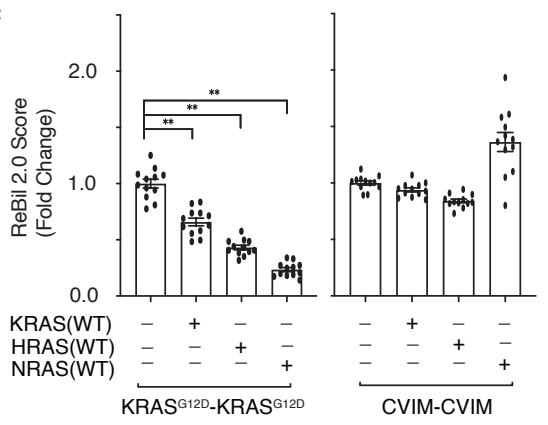

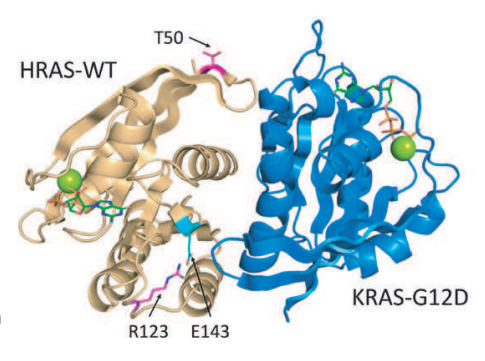

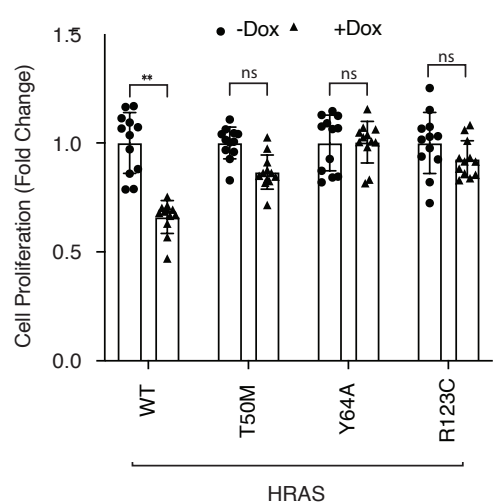

Figure 5. Wildtype RAS paralogs finetune RAS signaling through interaction with oncogenic KRAS.

a. Bubble plot of three AP/MS experiments with $\mathrm{H}-, \mathrm{K}-$, and $\mathrm{N}-\mathrm{RAS}$ as baits (rows), showing the enrichment of thier paralogs (columns).

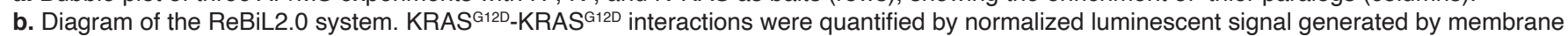
association facilitated interaction of the split-luciferase that is fused to the N-terminus of KRAS ${ }^{\mathrm{G} 12 \mathrm{D}}$ (upper). Split-luciferase that is fused to the last four amino acids of KRAS (CVIM) is applied as control for background split-luciferase interaction on the membrane (lower). Adapted from Li et al. 2020.

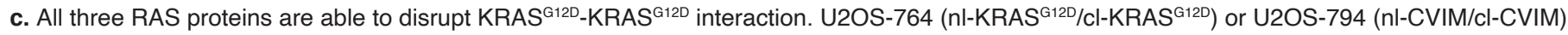
cells expressing KRAS, HRAS, or NRAS were cultured in limited serum (1\%) under $100 \mathrm{ng} / \mathrm{ml}$ Doxycycline (Dox) for 24 hours. ReBiL2.0 assay were performed as previously described and detailed in Methods. Points are Mean \pm SD ReBiL2.0 score of 36 wells normalized to cells transduced with empty lentiviral vector. ${ }^{* *}: p<0.01$

d. Pan-cancer frequency of HRAS mutations in patients with KRAS-wildtype and oncogenic KRAS-mutant tumors from Project GENIE. Known oncogenic HRAS mutations are highlighed in red. The dashed line indicates equal mutation frequency in KRAS-wildtype and mutant samples. Four candidate mutations that were chosen for further validation in this study were highlighted.

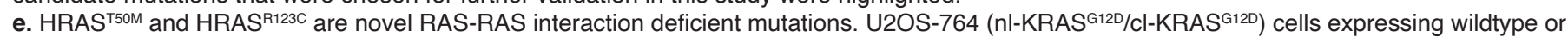
rare mutant HRAS were cultured in limited serum (1\%) under $100 \mathrm{ng} / \mathrm{ml}$ Dox for 24 hours. Points are Mean \pm SD ReBiL2.0 score of 12 wells normalized to cells transduced with empty lentiviral vector (upper). ${ }^{*}: \mathrm{p}<0.01$; ns: not significant. HRAS (wildtype and mutant) protein expression level in corresponding cells were shown by Western blot analysis (lower).

f. HRAS ${ }^{T 50 M}$ and HRAS ${ }^{\mathrm{R} 123 \mathrm{C}}$ are located close to the predicted HRAS-KRAS interaction interface. HRAS is shown in light orange and KRAS ${ }^{\mathrm{G} 12 \mathrm{D}}$ is shown in blue. Residue R123 (in magenta) makes an intrachain salt bridge with E143 (in cyan).

g. Prey RAF proteins enriched in each experiment with the indicated baits in A549 cells (for K-, H-, or N-RAS) or HEK293 cells (for KRAS). Both GTP- and GDP-bond HRAS behave like GDP-bond KRAS in their RAF interactions.

h. Western blot analysis of cultured HRAS-null HOP62 cells (HOP62-Cas9-sgHRAS) re-expressing wildytype or mutant HRAS (T50M, Y64A, or R123C) under Dox treatment. Cells were cultured under limited serum (1\%) for 2 days before protein extraction. Re-expression of HRAS mutants have no effects on ERK phosphrylation.

i. Cell proliferation of cultured HRAS-null HOP62 cells (HOP62-Cas9-sgHRAS) re-expressing wildytype or mutant HRAS (T50M, Y64A, or R123C) under Dox treatment. Cells werer cultured in limited serum (1\%) with or without Dox for 4 days. Cell viability was measured via CCK8 assay and normalized to cells treated with vehicle. Re-expression of HRAS mutants have no effects on cell proliferation. 


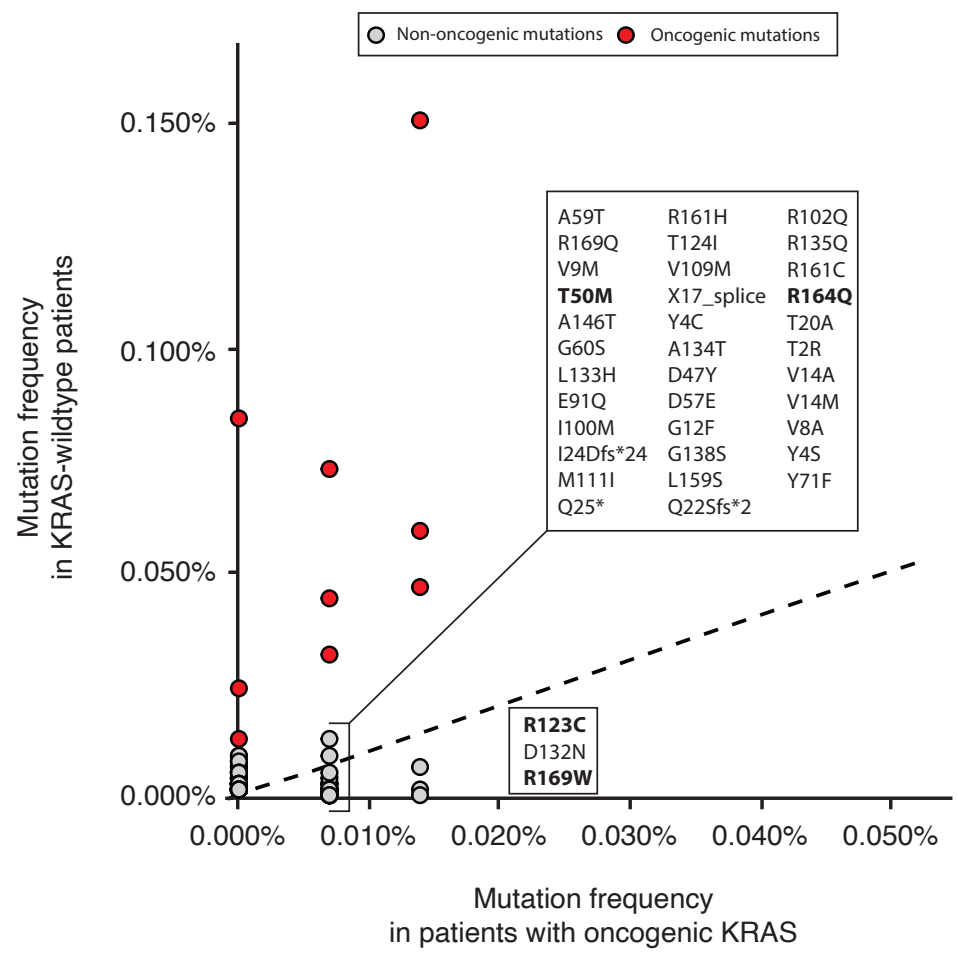

b

\begin{tabular}{|c|c|c|c|}
\hline Mutation & Cancer type & Sample type(s) & $\begin{array}{l}\text { Co-mutated with } \\
\text { oncogenic Kras? }\end{array}$ \\
\hline \multirow{4}{*}{ T50M } & Lung adenocarcinoma & Primary tumor, metastasis & Yes \\
\hline & Ampullary Carcinoma & Primary tumor & No \\
\hline & Leiomyosarcoma & Primary tumor & No \\
\hline & Malignant Fibrous Histiocytoma & Primary tumor & No \\
\hline \multirow{7}{*}{ R123C } & Rectal Adenocarcinoma & Primary tumor & Yes \\
\hline & Rectal Adenocarcinoma & Primary tumor & Yes \\
\hline & Colorectal Adenocarcinoma & Primary tumor & No \\
\hline & $\begin{array}{l}\text { Head and Neck Squamous } \\
\text { Cell Carcinoma }\end{array}$ & Primary tumor & No \\
\hline & Lung Adenocarcinoma & Primary tumor & No \\
\hline & Mixed Germ Cell Tumor & Primary tumor & No \\
\hline & Papillary Thyroid Cancer & Primary tumor & No \\
\hline $\mathrm{R} 164 \mathrm{Q}$ & Colorectal Adenocarcinoma & Metastasis & Yes \\
\hline \multirow{2}{*}{ R169W } & Colon Adenocarcinoma & Primary tumor & Yes \\
\hline & Colon Adenocarcinoma & Metastasis & Yes \\
\hline
\end{tabular}

Supplemental Figure 8. Identification of rare HRAS mutations in oncogenic KRAS-mutant tumors.

a. Pan-cancer frequency of HRAS mutations in patients with KRAS-wildtype and oncogenic KRAS-mutant tumors from Project GENIE. Mutations that are intergenic, intronic, silent, or fall in the 3' or 5' UTR were excluded. Oncogenic KRAS mutants were defined as tumors having missense mutations in codons 12,13 or 61 . Known oncogenic HRAS mutations are highlighted in red. The dashed line indicates equal mutation frequency in KRAS-wildtype and mutant samples. Non-oncogenic mutations occurring at least once in patients with

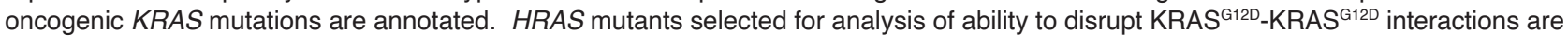
highlighted in bold.

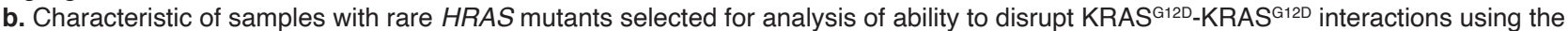
ReBiL2.0 system. 
a

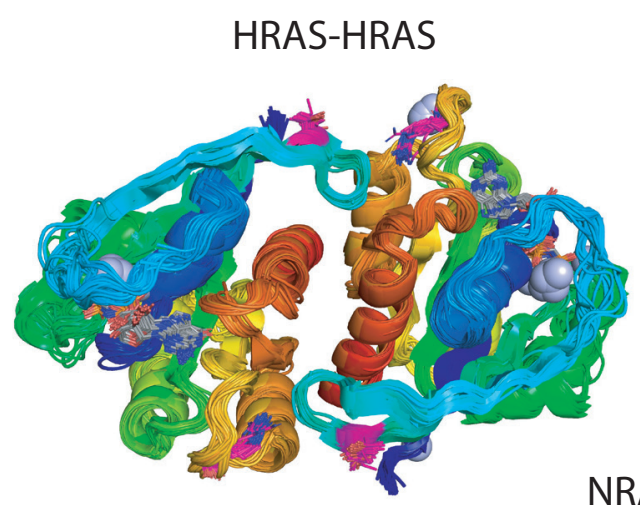

HRAS-HRAS

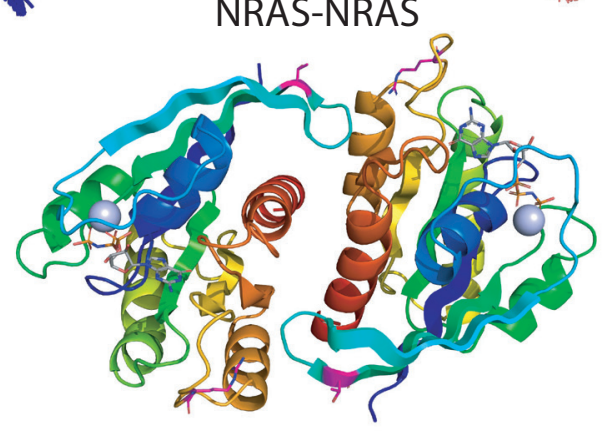

KRAS-KRAS

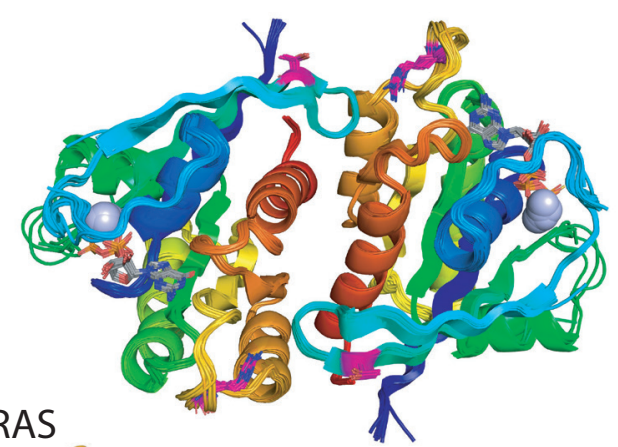

b
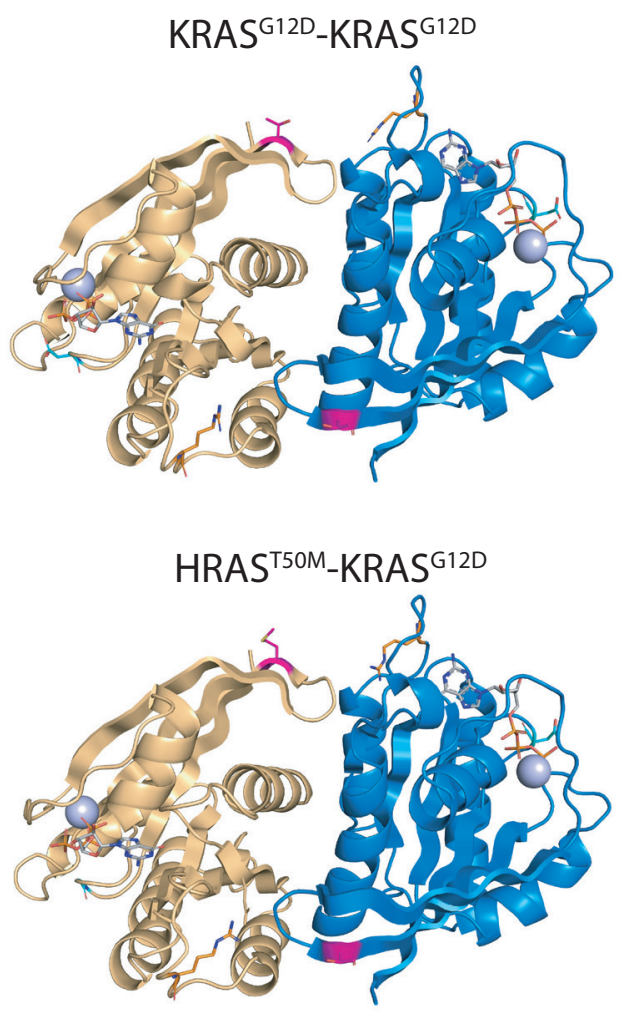
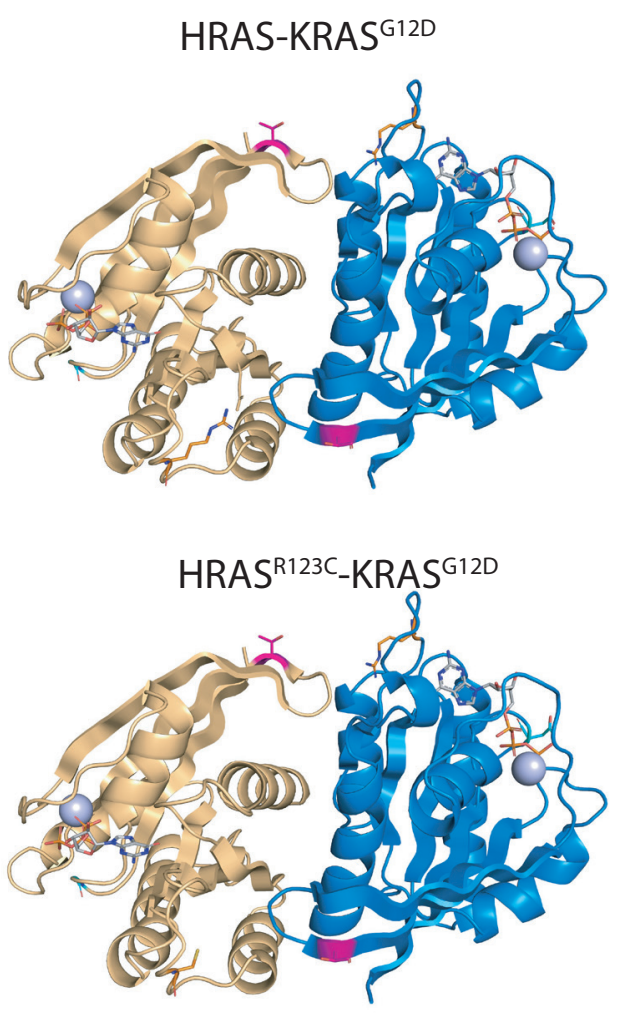

Supplemental Figure 9. Modeling RAS-RAS dimer.

a. Homodimers of RAS present in crystals of HRAS, KRAS, and NRAS in the Protein Data Bank. Dimers were downloaded from the Protein Common Interface Database (ProtCID), which clusters interfaces present in different crystals of homologous proteins. The a4-a5 dimer shown is present in 84 entries of HRAS, 13 entries of KRAS, and one entry of NRAS (PDB 5UHV).

b. Models of a homodimer of KRAS ${ }^{\mathrm{G} 12 \mathrm{D}}$ and heterodimers of KRAS ${ }^{\mathrm{G} 12 \mathrm{D}}$ with HRAS, HRAS ${ }^{\mathrm{T50M}}$, and HRAS ${ }^{\mathrm{R} 123 \mathrm{C}}$. The a4-a5 HRAS dimer from PDB entry $3 \mathrm{~K}^{2} 8 \mathrm{Y}$ was used as a template. KRAS ${ }^{\mathrm{G} 12 \mathrm{D}}$ from PDB entry $5 \mathrm{USJ}$ was superposed with the program PyMol on one or both monomers of $3 \mathrm{~K} 8 \mathrm{Y}$ to form the heterodimers and the homodimer respectively. Residues T50 and R123 were mutated with PyMol. All four structures were relaxed with the program Rosetta using the FastRelax protocol with the Ref2015 scoring function). Rosetta uses the backbone-dependent rotamer library of Shapovalov and Dunbrack to repack side chains around the mutated sites. The resulting energies were: KRAS ${ }^{\mathrm{G} 12 \mathrm{D}}$-KRAS ${ }^{\mathrm{G} 12 \mathrm{D}},-1122.8 \mathrm{kcal} / \mathrm{mol}$; HRAS-KRAS ${ }^{\mathrm{G} 12 \mathrm{D}},-1144.8$ kcal/mol; HRAS ${ }^{T 50}$-KRAS ${ }^{G 12 D},-1135.5$ kcal/mol; HRAS ${ }^{\text {R123C-KRAS }}{ }^{G 12 D}$, -1130.9 kcal/mol. Residues T50 (magenta) and R123 (orange) are indicated in sticks. 
a

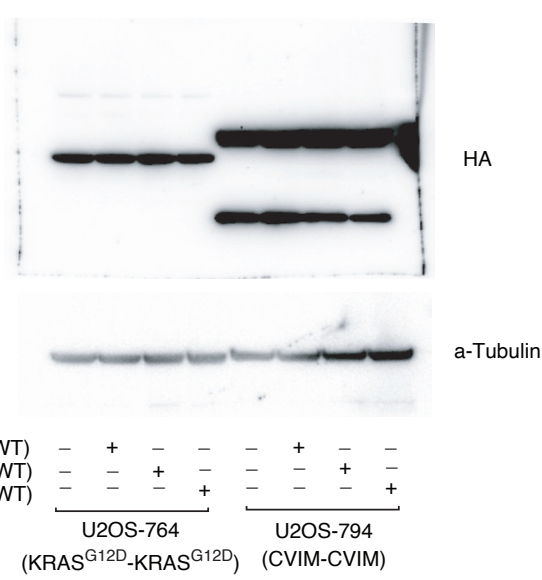

b

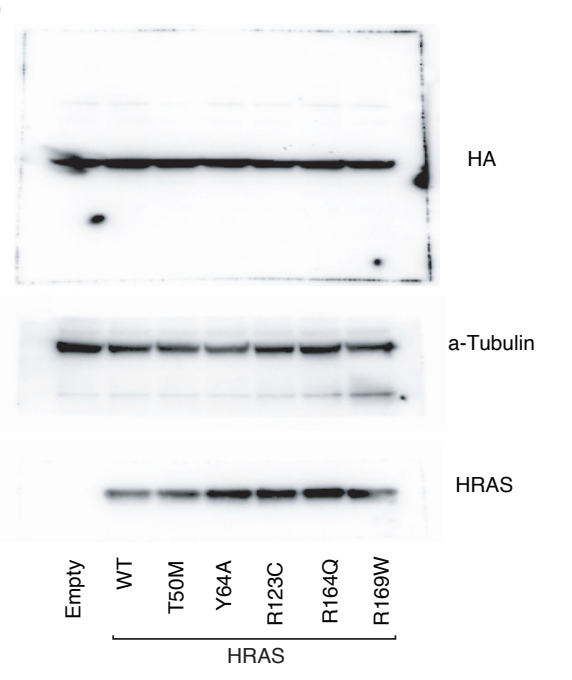

C
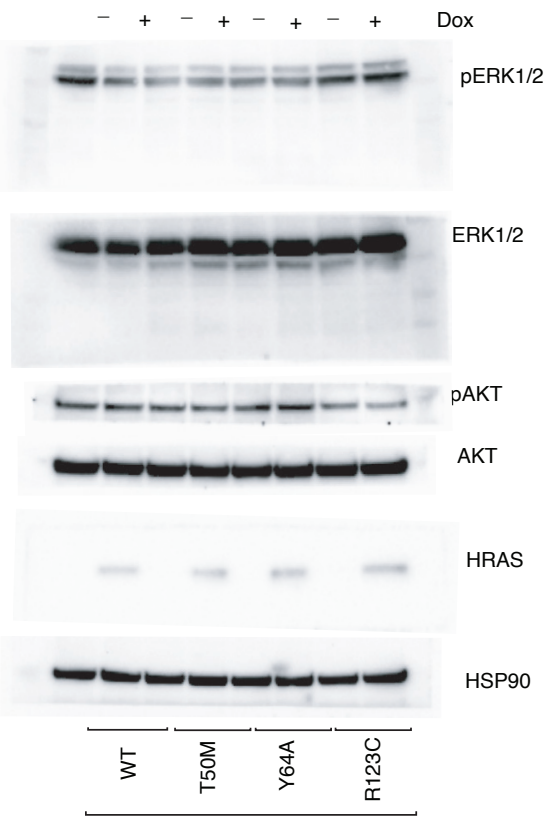

HRAS

Supplemental Figure 10. Wildtype RAS paralogs finetune RAS signaling through interaction with oncogenic KRAS.

a. Raw images for western blots of split-luciferase (HA-tag) expression for ReBiL2.0 from Figure 5c. HA-tag expression were detected using same lysis on a different gel with increased loading.

b. Raw images for western blots of split-luciferase (HA-tag) expression for ReBiL2.0 from Figure 5e. HA-tag expression were detected using same lysis on a different gel with increased loading.

c. Raw images for western blots from Figure 5h. HRAS expression were detected using same lysis on a different gel with increased loading. 


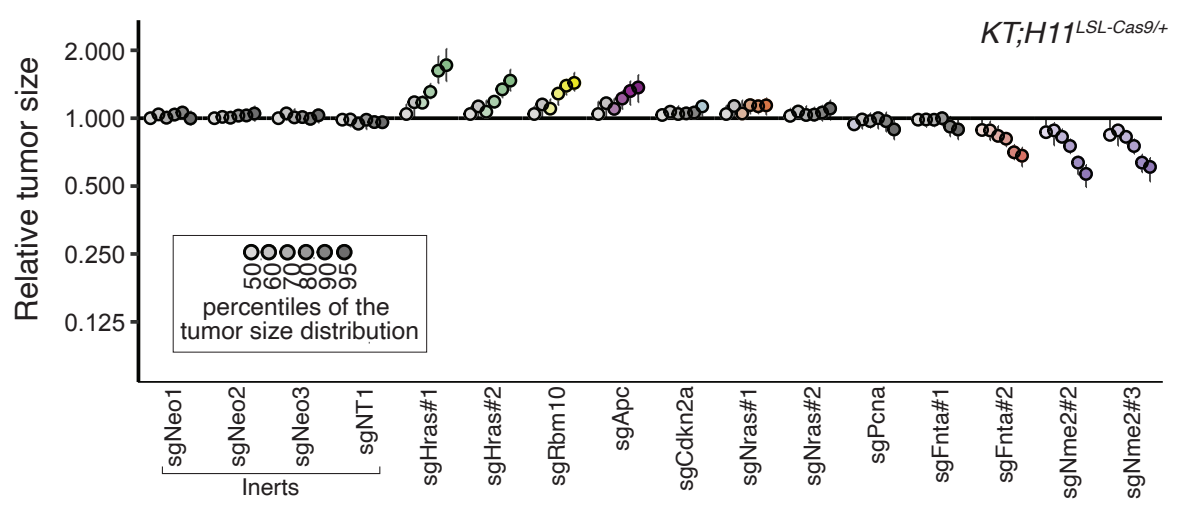

b

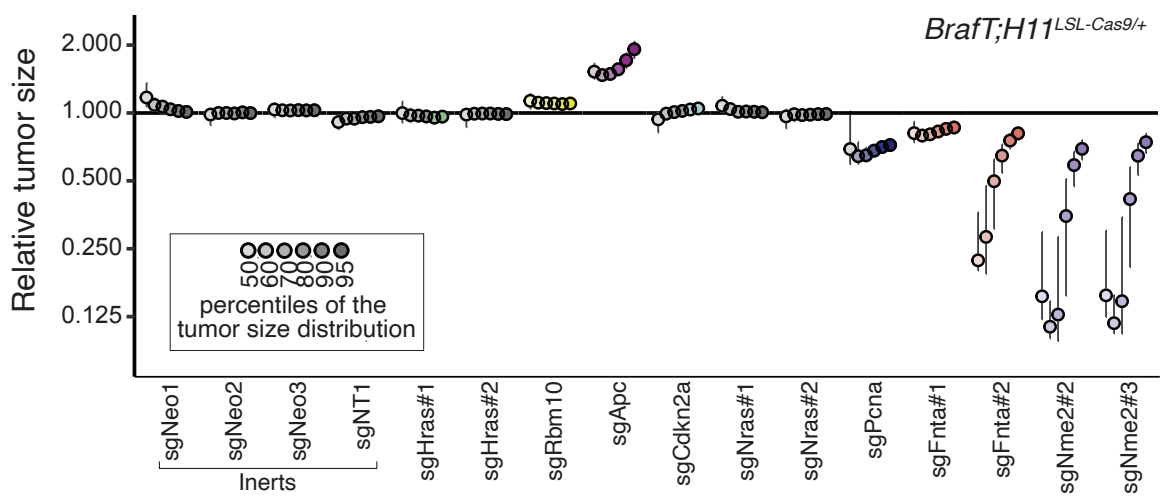

C

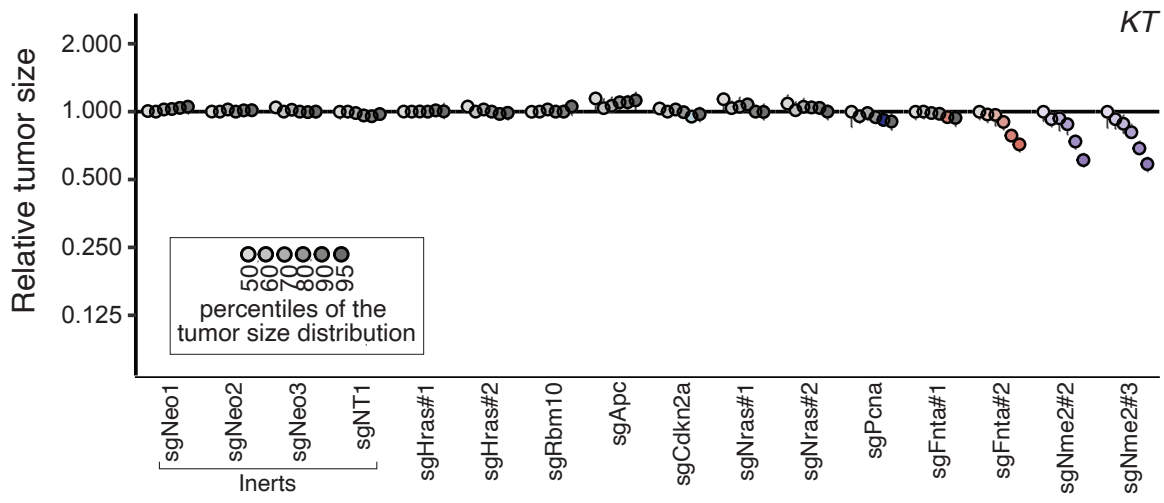

d
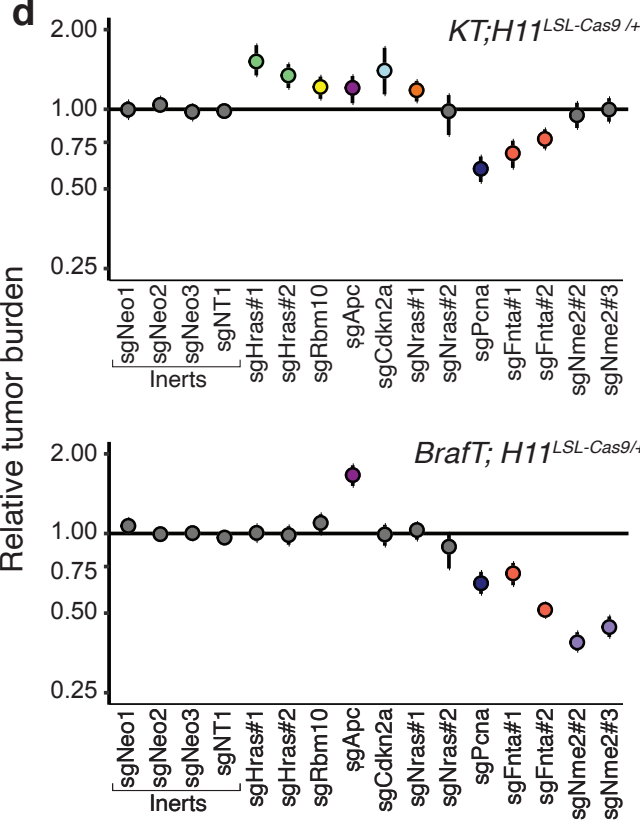

e

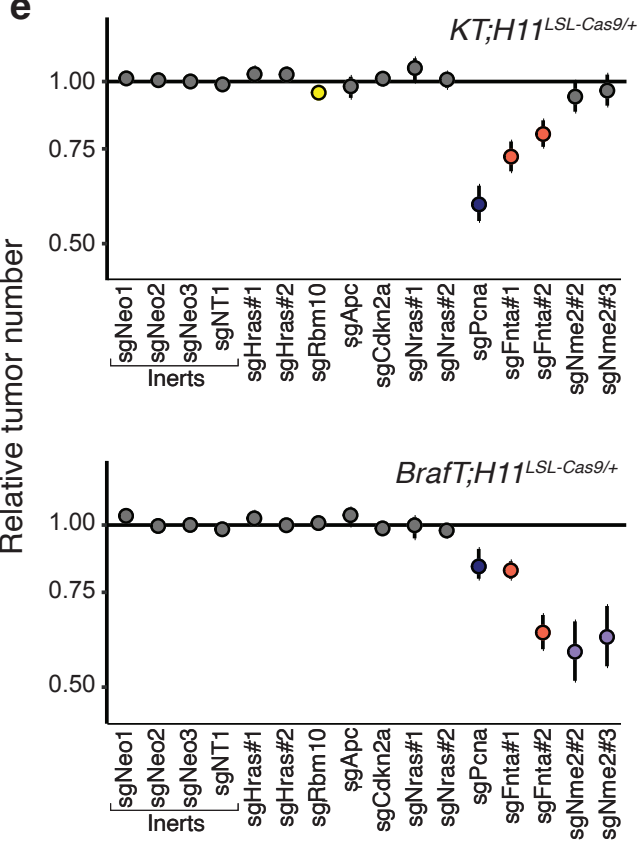

Supplemental Figure 11. Paired screen in KRAS-driven and Braf-driven lung cancer models validates Hras and Nras as Kras-specific tumor suppressors.

a-c. Tumor sizes at indicated percentiles for each sgRNA relative to the size of sglnert-containing tumors at the corresponding percentiles in $K T ; H 11^{L S L-C a s 9 /+}(\mathbf{a}), B r a f T ; H 11^{L S L-C a s 9 /+}(\mathbf{b})$ and $K T$ mice $(\mathbf{c})$. Genes are ordered by $95^{\text {th }}$ percentile tumor size in $K T ; H 11^{L S L-C a s 9 /+}$ mice, with sglnerts on the left. sglnerts are in gray, and line at $\mathrm{y}=1$ indicates no effect relative to sglnert. Error bars indicate $95 \%$ confidence intervals. Percentiles that are significantly different from sglnert (two-sided FDR-adjusted $p<0.05$ ) are in color. Confidence intervals and P-values were calculated by bootstrap resampling. The negative effects of sgRNAs targeting Fnta and Nme2 in the KT mice (c) are unexpected and indicate a potential bias in the size distributions of tumors with these genotypes. We note that the same bias may be present in the KTC and BrafTC data; however, sgRNAs targeting these genes in previous experiments showed consistent negative effects on tumor size, suggesting that the observed effects in this $K T C$ cohort are not solely the product of this bias.

d. The impact of each sgRNA on tumor burden relative to sglnerts in KT;H11LSL-Cas9/+ (top) and BrafT;H11 LSL-Cas9/+ (bottom) mice, normalized to the corresponding statistic in KT mice to account for representation of each sgRNA in the viral pool. sglnerts are in gray and the line at $y=1$ indicates no effect. Error bars indicate 95\% confidence intervals. Relative tumor burdens significantly different from sglnert (two-sided FDR-adjusted $\mathrm{p}<0.05$ ) are in color. Confidence intervals and $\mathrm{P}$-values were calculated by bootstrap resampling.

d. The impact of each sgRNA on tumor number relative to sglnerts in KT;H11LSL-Cas9/+ (top) and BrafT;H11 LSL-Cas9/+ (bottom) mice, normalized to the corresponding statistic in KT mice to account for representation of each sgRNA in the viral pool. sglnerts are in gray and the line at $\mathrm{y}=1$ indicates no effect. Error bars indicate $95 \%$ confidence intervals. Relative tumor numbers significantly different from sglnert (two-sided FDR-adjusted $\mathrm{p}<0.05$ ) are in color. Confidence intervals and P-values were calculated by bootstrap resampling. 


\section{Supplementary Files}

This is a list of supplementary files associated with this preprint. Click to download.

- SupplementaryTable1.xIsx

- SupplementaryTable2.xlsx 